DE-FG05-88

DOE/ER/ 40 451--4

DE92 016459

\title{
MEASUREMENTS OF OBSERVABLES IN THE PION-NUCL.EON SYSTEM, NUCLEAR A-DEPENDENCE OF HEAVY QUARK PRODUCTION AND RARE DECAYS OF D AND B MESONS
}

\author{
Progress Report \\ 1 December, 1990 - 15 February, 1992
}

Michael E. Sadler and L. Donald Isenhower

Abilene Christian University

Abilene, TX 79699

15 February, 1992

PREPARED FOR THE U.S. DEPARTMENT OF ENERGY UNDER GRANT NI IMBER DE-FG05-88ER40451 
Table of Contents (Progress Report)

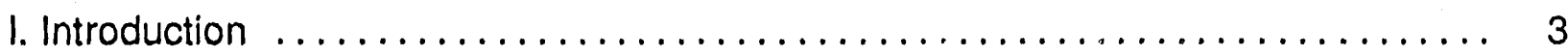

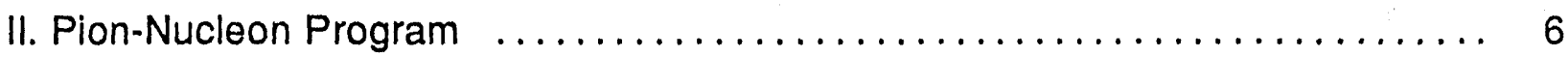

A. Complete Sets of Measurements at Plab $=427$ to $657 \mathrm{MeV} / \mathrm{c} \quad \ldots \ldots \ldots$... 6

B. Measurements of $\pi-p \rightarrow \pi \circ$ Using the LAMPF $\pi$ o Spectrometer $\ldots \ldots \ldots 13$

1. Experiment 882: Low Energy Single Charge Exchange ........ 13

2. Experiment 849: Medium Energy Single Charge Exchange .... 22

C. Proposed Program with the New Neutral Meson Spectrometer ....... 22

D. $\pi N$ Partial-Wave Analysis and the $\pi N N$ Coupling Constant .......... 44

E. Measurement of the Differential Cross Sections for $i \cdot p \rightarrow \pi \circ$ and $\pi \cdot p \rightarrow \eta n$ at the St. Petersburg Nuclear Physics Institute $\ldots \ldots \ldots \ldots, 46$

1. Summary of Experimental Program $\ldots \ldots \ldots \ldots \ldots \ldots \ldots \ldots, 46$

2. Feasibility Study of $\eta$ Production $\ldots \ldots \ldots \ldots \ldots \ldots \ldots \ldots, 48$

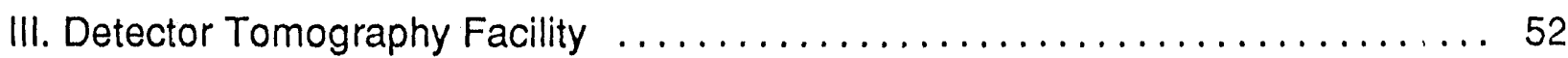

IV. FNAL E789: Nuclear Dependence of Charm and Beauty Quark Production and a Study of Two-Prong Decays of Neutral D and B Mesons ......... 58

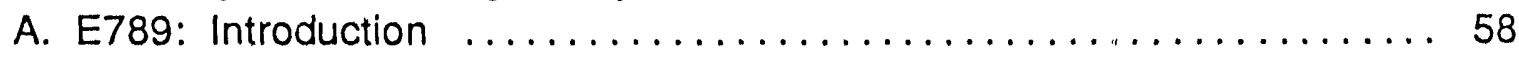

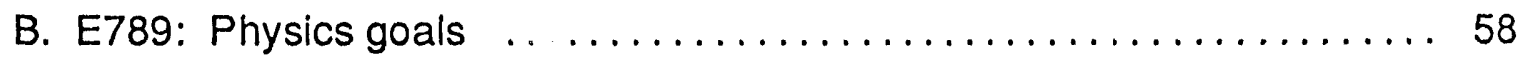

C. Future of E789 Collaboration and Summary $\ldots \ldots \ldots \ldots \ldots \ldots \ldots, 61$

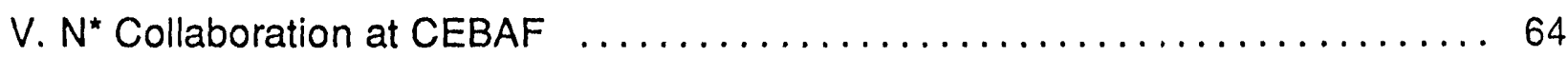

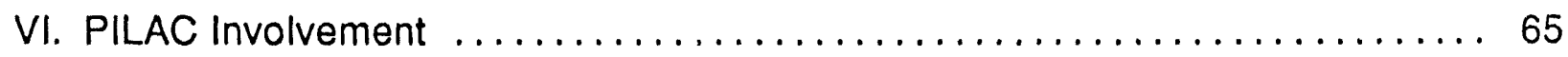

A. Overview of Physics Goals $\ldots \ldots \ldots \ldots \ldots \ldots \ldots \ldots \ldots \ldots \ldots \ldots \ldots$

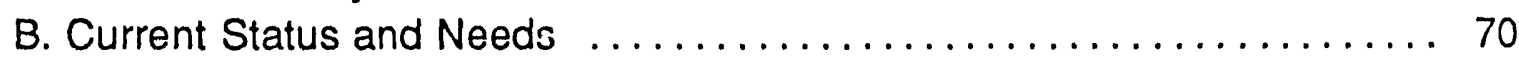

C. Advantages of PILAC .............................. 71

D. Examples on Improvements PILAC Can Make on $\pi \mathrm{N}$ Observables .... 72

E. Design Work on the Superconducting Quadrupoles for PILAC $\ldots \ldots \ldots 75$

VII. Report on leave of absence for L.D. Isenhower $\ldots \ldots \ldots \ldots \ldots \ldots \ldots, 78$

VIII. Time Schedule $\ldots \ldots \ldots \ldots \ldots \ldots \ldots \ldots \ldots \ldots \ldots \ldots \ldots \ldots, \ldots \ldots$

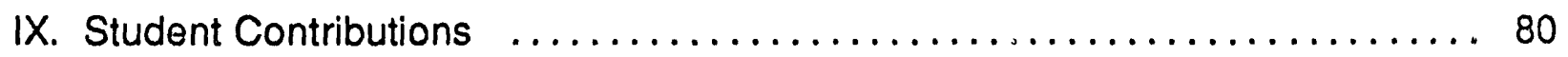

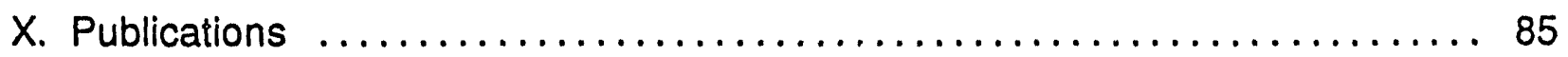

Leprint removed 2 


\section{Introduction}

This report summarizes the progress by the ACU particle physics research group under this grant number DE-FG05-88ER40451 from the U.S. Department of Energy from 1 December 1990 to the present. The experiments to date have been conducted at the Clinton P. Anderson Meson Physics Facility at Los Alamos (LAMPF), Fermilab, and the Leningrad (now Saint Petersburg) Nuclear Physics Institute.

Highlights of the accomplishments in this program since renewal in 1990 include:

- Fermilab Experiment 789, completion of data collection in January, 1992. This experiment has a primary emphasis on the measurements of the two-prong decays of $B$ and D mesons ( J.C. Peng, LANL and D.H. Kaplan, NIU, spokespersons). On E789 ACU is collaborating with P2 Division of the Los Alamos National Laboratory, Fermilab, Northern Illinois University, University of Chicago, University of California at Berkeley, University of South Carolina, and Academia Sinica, Taiwan.

- A collaboration between what is now called the St. Petersburg Nuclear Physics Institute (SPNPI), UCLA and ACU to measure observables for $\pi \cdot p \rightarrow \pi$. threshold was begun. The production of the $\eta$ meson from the reaction $\pi \cdot p \rightarrow \eta n$ was identified for the first time at the laboratory during a two-week tune-up run in July, 1991. The results have been analyzed at ACU as a mechanism to measure the central beam momentum and momentum bite $(\triangle P / P)$ of the SPNPI pion beam, an important facet of the measurement of $\pi \cdot p \rightarrow \pi^{\circ}$ n near the $\eta$ threshold.

- Continued effort in the analysis and publication of results for the polarization observables in the $\pi \mathrm{N}$ system which were measured prior to 1988. The final paper for Experiment 804, entitled Measurement of the Left-Right Asymmetry in $\pi \cdot p \rightarrow m$ from 301 to $625 \mathrm{MeV} / \mathrm{c}$ at the Backward Angles, was published in Phys. Rev. D. The final paper for Experiment 806 , measurement of the spin rotation parameters $A$ and $R$ in $\pi \mathrm{N}$ scattering from 427 to $657 \mathrm{MeV} / \mathrm{c}$, has just been submitted for publication (an article in Physical Review Letters has already been published). These data finish the program to obtain the first complete sets of measurements (defined in Sec. II) for the $\pi \mathrm{N}$ system in any momentum interval. The attainment of this goal culminates a program that was started in 1978, when the cross sections for elastic scattering, $\pi \pm p \rightarrow \pi \pm p$, were measured.

- Publication of a work, Comment on "Determination of the $\pi N N$ Coupling Constant from Elastic Pion-Nucleon Scattering Data", Phys. Rev. Lett., 68, 548 (1992). This paper was a product of a month-long (August, 1990) visit to ACU by two eastern European physicists, J. Stahov from Tuzla, Yugoslavia, and V. Abaev from St. Petersburg. A collaborative effort toward a new partial wave analysis of the 
existing $\pi \mathrm{N}$ data is continuing despite the political uncertainties in both of those countries.

- Report on the first application of an equality test of isospin invariance in the $\pi \mathrm{N}$ system at the IVth International Symposium on Pion-Nucleon Scattering at Bad Honnef, Germany in September, 1991. This work is another result of the collaboration with Stahov and with $\mathrm{G}$. Höhler and $\mathrm{H}$. Staudenmaier of Karlsruhe.

- Visit of Cvjetan Jakovljevich, a Yugoslav graduate student with Stahov, who spent three months in Los Alamos and three months in Abilene working on the LAMPF experimental program, particularly the analysis of Exp. 849, as a thesis project. His health and the political problems in Yugoslavia forced him to return to Tuzla in November. Future collaboration with this group and with Ivan Supek from Rudjer Boskovic Institute in Zagreb is anticipated through a joint U.S.-Yugoslav grant for collaborative science between the countries.

- LAMPF Experiment 849, transfer of responsibility of analysis to another GWU student for his Ph.D thesis after the completion of a master's thesis by N.J. Nicholas, a George Washington University student. This experiment measured the differential cross section for $\pi \cdot p \rightarrow \pi \circ n$ near 00 and 1800 in the momentum region 427-687 MeV/c (M. Sadler, spokesperson).

- The graduation of the first student under a cooperative agreement with Texas Tech University under which Isenhower and Sadler are appointed adjunct faculty in order to supervise graduate theses. Meade Brooks completed his Master's thesis on LAMPF Experiment 882, measurement of differential cross sections for $\pi \cdot p \rightarrow \pi \circ$ at 10,20 and $40 \mathrm{MeV}$ near 00 and $180^{\circ}$. The results were reported by Sadler the IVth Int. Symp. on $\pi N$ Scattering at Bad Honnef.

- LAMPF Experiment 1129, publication of final results (Phys. Rev. C44, 2869(1991)). This experiment was a search for neutral pions from the spontaneous fission of $252 \mathrm{Cf}$ (J. Knudson, Los Alamos, spokesperson). The collaboration included scientists from LANL and ACU. The $\pi$ o spectrometer was set up in the $\mathrm{P}^{3}$ channel to detect low-energy pions from a $5.8 \mathrm{mCi} 252 \mathrm{Cf}$ source for 893 hours (source in) and 354 hours (source out) in the late summer and fall of 1988 after Exp. 849. The only beam time required for the measurement was a short run in which a $160 \mathrm{MeV}$ $\pi^{-}$beam was stopped in a $2.5 \mathrm{~cm}$ polyethylene target after passing through a $60 \mathrm{~cm}$ graphite degrader. Detection of the low-energy neutral pions from $\pi-p \rightarrow \pi \circ n$ confirmed that the $\pi^{\circ}$ spectrometer was operating properly. An upper limit of $1.4 \mathrm{x}$ 10-11 was obtained for the branching ratio of $\pi 0^{\prime}$ s produced at rest.

- Return of Isenhower from a one-year leave of absence at CERN working on the DELPHI detector funded by an NSF/NATO Postdoctoral Fellowship.

- Participation by Sadler on the committee for the design and implementation of the Neutral Meson Spectrometer (NMS) at Los Alamos. Meetinç have been held at two-three month intervals. ACU students assisted in the initial setup and operation 
of the tomography apparatus being used to test parts of the new Neutral Meson Spectrometer nearing completion at LAMPF.

- Continued work by Sadler as co-chair (with W. Gibbs of New Mexico State University) of the baryon resonance working group for writing the physics justification for PILAC (Eion Linear Accelerator) at LAMPF.

- Continued quality involvement by ACU undergraduate students in this research effort, particularly in data analysis and in preparing for experiments. 


\section{Pion-Nucleon Program}

\section{A. Complete Sets of Measurements at $\mathrm{P}_{\mathrm{lab}}=427$ to $657 \mathrm{MeV} / \mathrm{c}$}

Abilene Christian University has been involved in pion-nucleon $(\pi \mathrm{N})$ scattering experiments at the Clinton P. Anderson Meson Physics Facility at Los Alamos (LAMPF) since 1979. The experiments have been conducted in collaboration with UCLA, George Washington University, Los Alamos, Catholic University and Rudjer Boskovic Institute.

The goal from the "outset of this program was to obtain the first complete set of measurements ever in the fundamental $\pi \mathrm{N}$ system. That goal has been accomplished. The experimental phase in the momentum region from 378 to $687 \mathrm{MeV} / \mathrm{c}$ has come to a close. Measurements made at momenta from 378 to $687 \mathrm{MeV} / \mathrm{c}$ in the $\mathrm{P}_{3}$ channel as part of the $\pi \mathrm{N}$ scattering program consist of the following (years in which the data taking phase was completed are given in parentheses):

- differential cross sections for $\pi+p \rightarrow \pi+p$ and $\pi-p \rightarrow \pi-p$, LAMPF Experiment $363(1979)^{1}$.

- differential cross sections for pion-nucleon charge exchange, $\pi \cdot p \rightarrow \pi \circ n$, LAMPF Experiment $120(1980)^{2}$,

- polarization asymmetry for elastic scattering, $\pi^{ \pm} p \rightarrow \pi^{ \pm} p$, LAMPF Experiment $120^{*}(1982)^{3}$,

- polarization asymmetry for charge exchange, $\pi-p \rightarrow \pi \circ n$, LAMPF Experiment $120^{\star \star}(1982) 4$,

- polarization asymmetry for radiative capture, $\pi \cdot p \rightarrow \gamma n$, and a high precision measurement near 900 of the polarization asymmetry for $\pi$-p $\rightarrow \pi$ \%n, LAMPF Experiment $804(1984)^{5}$,

- proton polarization from $\pi p$ elastic scattering at 547 and $625 \mathrm{MeV} / \mathrm{c}$, LAMPF Experiment 807, (1985)6,

- measurement of the spin rotation parameters $A$ and $R$ for $\pi^{ \pm} p \rightarrow \pi^{ \pm} p$ elastic scattering, LAMPF Experiment 806 (1986)7, and

- differential cross sections for $\pi-p \rightarrow \pi$ on near 00 and 1800 in the momentum region 471-687 MeV/c, LAMPF Exp. 849 (1988)8.

Extensive discussion of these experiments, particularly the test of isospin invariance in the $\pi \mathrm{N}$ system made possible by a complete set of measurements, was included in the 1990 progress report and is not reproduced here. The main progress made in the past year in this program has been the submission of the final publication7 of the spin rotation measurements. The error analysis was a major area of concern in presenting the final results to the physics community since these measurements are not likely to be repeated in the foreseeable future. The errors were made more conservative to account for differences at some angles between results obtained with 
opposite target polarizations. The differences were attributed to instrumental uncertainties in the proton polarimeter (JANUS). The experiment was designed so that these uncertainties canceled when averaged over both target polarizations. A global analysis of the data indicated that the rms deviation of the $A$ and $R$ parameters between the target polarizations was 0.13 . This uncertainty was added in quadrature to all the data, resulting in doubling some of the errors (the smallest errors of 0.07 were increased to 0.15 , while the larger errors of 0.2 or more were largely unaffected).

The A-and-R data in Fig. 1-2 at $547 \mathrm{MeV} / \mathrm{c}$ have been updated to reflect the increased error bars. Also shown are differential cross sections and analyzing powers from Exp. 363 and 120*, respectively. These results combined with the differential cross sections and analyzing powers for $\pi \cdot p \rightarrow \pi^{\circ}$ n (Exp. 120 and $120^{* *}$, shown in the 1990 progress report) make a total of ten observables for seven independent amplitudes. These data are now the world standard for a compiete data set (defined as enough observables to determine fully the isospin and spin amplitudes) in any hadronic system.

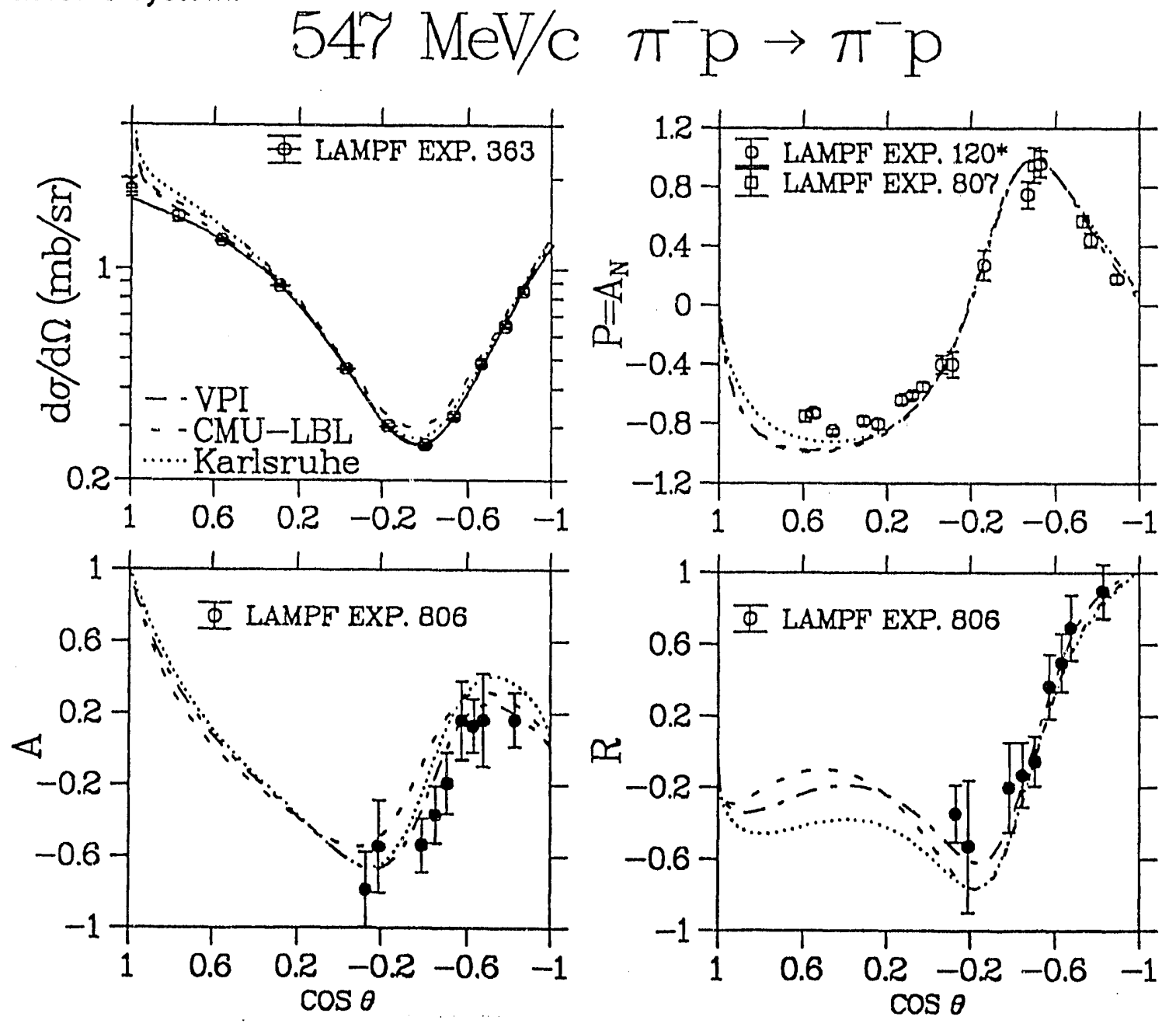

Figure 1 

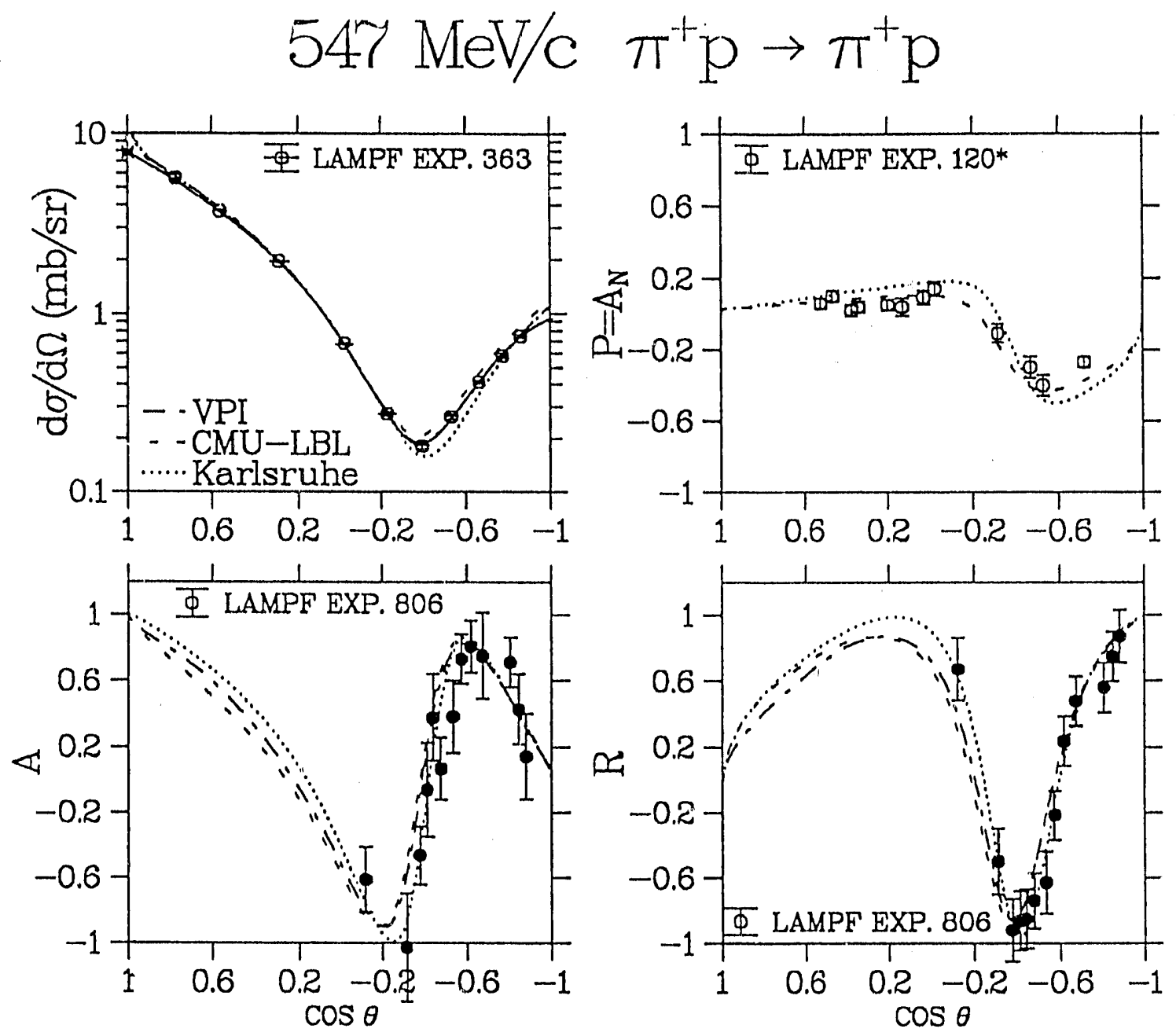

Figure 2

The following contribution, submitted to the IVth Pion-Nucleon Workshop held in Bad Honnef, Germany in September, 1991, describes the application of the complete data sets to test isospin invariance.

\section{References:}

1. M. E. Sadler, et al., Phys. Rev. D35, 2718 (1987).

2. F. O. Borcherding, UCLA thesis, 1982 (unpublished).

3. A. Mokhtari, et al., Phys. Rev. D35, 810 (1987).

4. J. A. Wightman, et al., Phys. Rev. D36, 3529 (1987).

5. G. J. Kim, et al., Phys. Rev. Lett. 56, 1779 (1986), and Phys. Lett. B219, 62 (1989), and accepted for publication in Phys. Rev. D.

6. C. J. Seftor, et al., Phys. Rev. D39, 2457 (1989).

7. I. Supek, et al., submitted to Phys. Rev. D and D. B. Barlow, et al., Phys. Rev. Lett. 62, 1009 (1989). 


\title{
EQUALITY TEST OF ISOSPIN INVARIANCE USING COMPLETE DATA SETS FOR PION-NUCLEON SCATTERING
}

\author{
M. E. Sadler \\ Abilene Christian University, Abilene, TX 79699
}

\begin{abstract}
An equality test of isospin invariance is possible for the first time in the $\pi \mathrm{N}$ system due to the complete data sets obtained at LAMPF. This test is described and presented at $P_{\pi}=547 \mathrm{MeV} / \mathrm{c}$.

Simply stated, isospin invariance dictates that the $\pi \mathrm{N}$ interaction depends on only two isospin amplitudes $(I=1 / 2,3 / 2)$ possible from the interaction of the pion (which has $I=1)$ with the nucleon $(I=1 / 2)$, with the contributions from these independent amplitudes determined from the Clebsch-Gordan coefficients of angular momentum coupling. Since three different charge couplings can be measured in $\pi N$ elastic scattering, namely $\pi+p \rightarrow \pi+p, \pi-p \rightarrow \pi \cdot p$ and $\pi \cdot p \rightarrow$ $\pi$, isospin invariance leads to distinct relationships derived from the observables in these reactions. Isospin invariance can be tested using the complete data sets obtained at LAMPF by the UCLA-ACU-GWU collaboration ${ }^{1-7]}$, consisting of differential cross section and analyzing powers for all three reactions and the spin rotation parameters $A$ and $R$ for the charged particle final states. The author is indebted to the Karlsruhe group8] for instruction in relating the experimental observables in such a way that isospin can be tested.
\end{abstract}

Fig. 1 shows how isospin invariance in the $\pi \mathrm{N}$ system can be tested if the differential cross section $(\mathrm{d} \sigma / \mathrm{d} \Omega$ ) and polarization asymmetry $(\mathrm{P})$ are measured for all three reactions and the spin rotation angles, $\beta^{+}$and $\beta$, are measured for $\pi^{+} p \rightarrow \pi^{+} p$ and $\pi \cdot p \rightarrow \pi \cdot p$, respectively. $\beta$ is related to $A$ and $R$ by the relation

$$
\beta=-\tan ^{-1}(-A / R)+\theta_{N}+\theta,
$$

where $\theta_{N}$ is the laboratory angle of the recoil nucleon (the proton) and $\theta$ is the center-of-mass angle of the scattered pion. The squares of the magnitudes of the transversity amplitudes, $[F( \pm) \mid 2$, are determined from the scattering observables by 


$$
|F( \pm)|^{2}=\frac{d \sigma}{d \Omega}(1 \pm P)
$$

$P$, the recoil nucleon polarization, is equal to $A_{N}$, the scattering asymmetry from a polarized target, by time reversal. The combination using the + sign is commonly called the transversityup amplitude and the one with the - sign is called the transversity-down amplitude. The subscripts used for $F(t)$ in Fig. 1 correspond to the different charge states of the reaction. $F_{+}(t)$ is the transversity-up amplitude for $\pi+p \rightarrow \pi+p, F_{0}(t)$ is the transversity-up amplitude for $\pi \cdot \mathrm{p} \rightarrow \pi \circ \mathrm{n}$, etc. Finally, the interdependence of the scattering amplitudes for the three reactions (shown in Fig. 1) is

$$
\sqrt{2} F_{0}( \pm)=F_{+}( \pm)-F_{\cdot}( \pm)
$$

forming two triangles in isospin space. Knowing the magnitudes (determined from experimental data, Ref. 1-7) of all three of these amplitudes allows one to determine the angle, $\gamma(t)$, between $F_{+}(t)$ and $F_{-}(t)$, using the law of cosines. A similar determination is possible for $\gamma(-)$ using the transversity-down amplitudes. The relative orientations of the two transversity amplitudes for $\pi^{+} p$ scattering is $\beta_{+}$, and for $\pi$-p scattering is $\beta_{\text {. }}$ Finally, isospin invariance can be tested using the relation

$$
\beta_{+}-\beta_{.}= \pm \gamma(+) \pm \gamma(-)
$$

if $d \sigma / d \Omega$ and $P$ are known for all three reactions and $\beta_{+}$and $\beta$. for the elastic reactions are known at a given energy and angle. The sign ambiguities result from only being able to determine the magnitudes of $\mathrm{F}_{+,-, 0}$ from experimental data alone.

Using the complete data sets from the UCLA-ACU-GWU collaboration, the quantities on each side of the previous equation are plotted in Fig. 2, again at $547 \mathrm{MeV} / \mathrm{c}$. The points with error

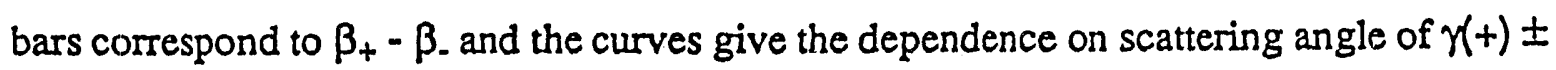
$X(-)$ based on fits to $d \sigma / d \Omega$ and $P$. The points lie on the curves, justifying the application of the principle of isospin invariance. The points actually change curves at $\cos \theta=-0.5$, a phenomenon caused by saturation of one of the isospin triargles, which corresponds to the triangle having zero area. The degeneracy of the curves near $\cos \theta=0.4$ arises from the fits to 
the cross section and polarization data violating the bounds allowed by the triangle inequality but is of little consequence here since this angle is outside the region where we have accurate measurements for $A_{N}(=P)$ for the charge exchange reaction.

1. M. E. Sadler, et al., Phys. Rev. D35, 2718 (1987).

2. F. O. Borcherding, UCLA thesis, 1982 (unpublished).

3. A. Mokhtari, et al., Phys. Rev. D35, 810 (1987).

4. J. A. Wightman, et al., Phys. Rev. D36, 3529 (1987).

5. G. J. Kim, et al., Phys. Rev. D41, 733 (1990).

6. C. J. Seftor, et al., Phys. Rev. D39, 2457 (1989).

7. D. B. Barlow, et al., Phys. Rev. Lett. 62, 1009 (1989) and I. Supek, et al., to be publ.

8. G. Höhler, A. Schulte, J. Stahov and H. M. Staudenmaier, Universität Karlsruhe, private communication.

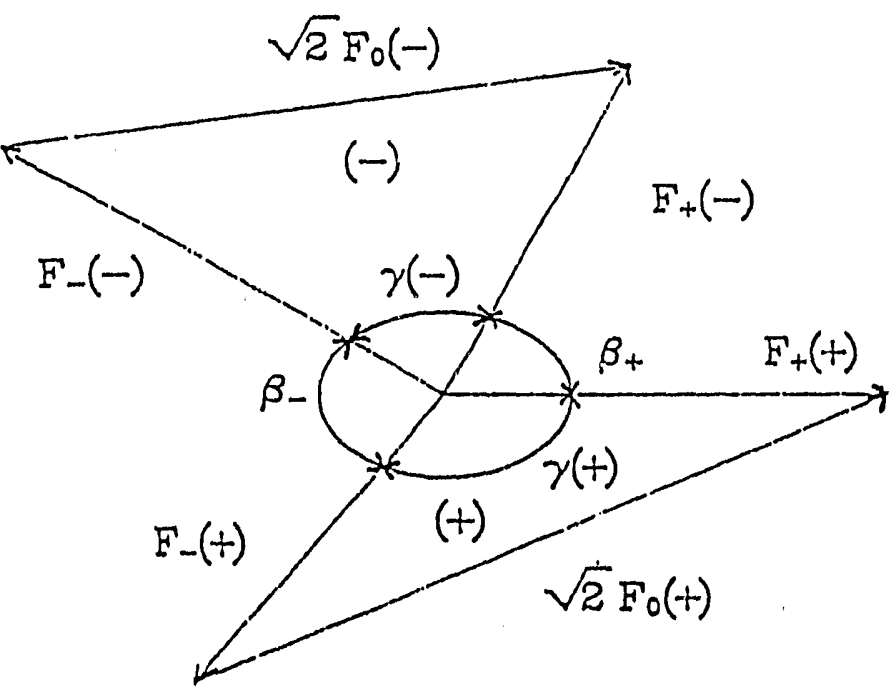

$$
\begin{aligned}
& \left|F_{+}(t /-)\right|^{2}=\mathrm{d} \sigma^{+} / \mathrm{d} \Omega\left(1+/-\mathrm{A}^{+} \mathrm{N}\right) \\
& \left|F_{-}\left(t^{\prime}-\right)\right|^{2}=\mathrm{d} \sigma^{-} / \mathrm{d} \Omega\left(1+/-\mathrm{A}^{-} \mathrm{N}\right) \\
& \left|F_{0}(t /-)\right|^{2}=\mathrm{d} \sigma^{0} / \mathrm{d} \Omega\left(1+/-\mathrm{A}^{0} \mathrm{~N}\right)
\end{aligned}
$$

Figure 1: The two isospin triangles and their relationship to experimental data. 


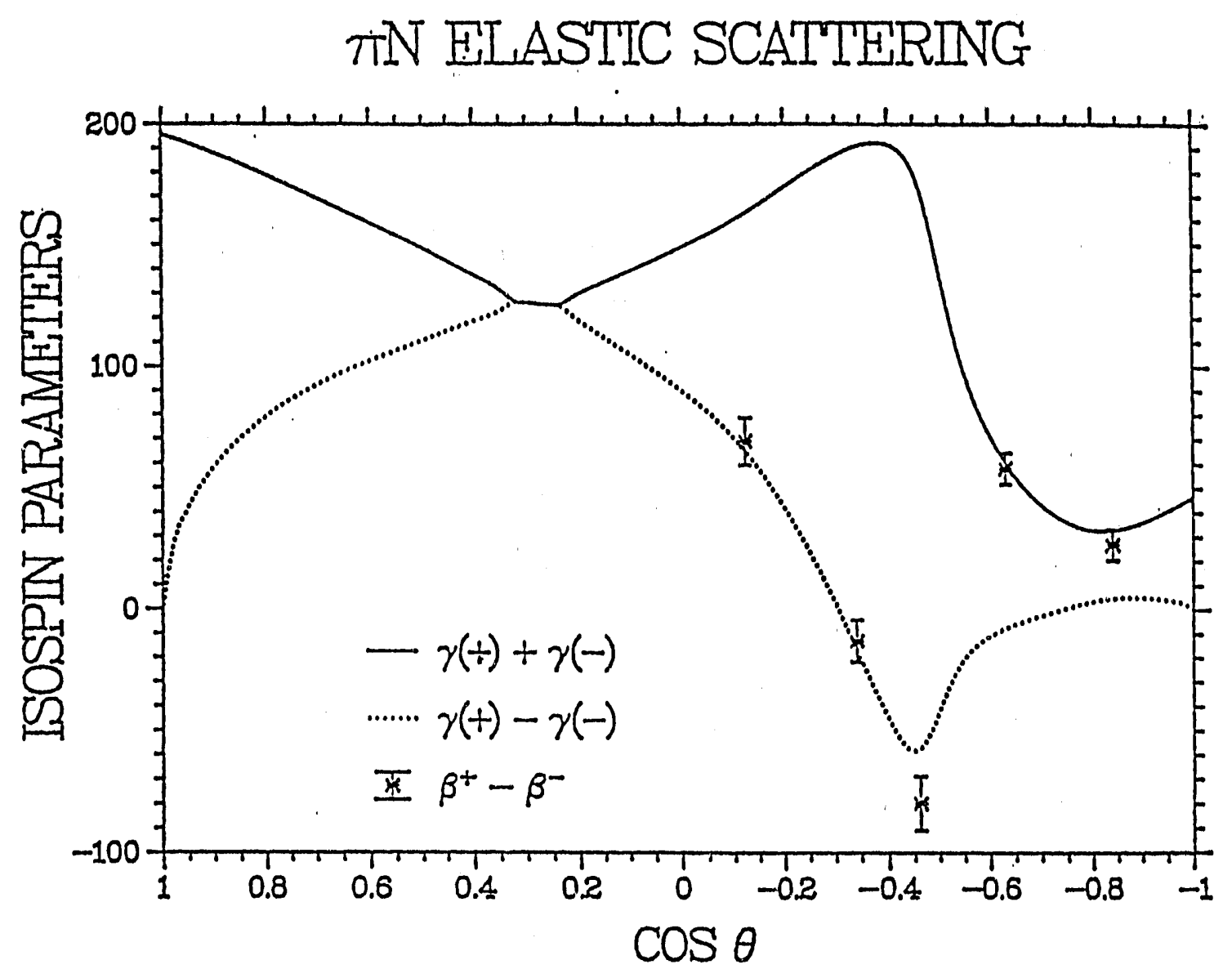

Figure 2: Comparison of the difference of the spin rotation angles (points with error bars) with what is allowed by isospin invariance at $547 \mathrm{MeV} / \mathrm{c}$. The curves are obtained from fits to the differential cross sections and analyzing powers. 


\section{B. Measurements of $\pi \cdot p \rightarrow \pi$ on Using the LAMPF $\pi^{\circ}$ Spectrometer}

\section{Experiment 882: Low Energy Single Charge Exchange}

James Redmon and Meade Brooks contributed to this section.

LAMPF Experiment 882, measurements of the differential cross sestions for $\pi \cdot p \rightarrow \pi \circ$ at $0^{\circ}$ and $180^{\circ}$ at $T_{\pi}=10,20$, and $40 \mathrm{MeV}$, is nearly ready for publication. Abso'ute efficiencies have been determined for the multi-wire proportional chambers and $\mathrm{Pb}$-glass converters in the LAMPF PIO spectrometer. In order to measure the differential cross sections to the $5 \%$ level, the efíciency of the detector must be known to that accuracy. The total efficiency is a function of the wire chamber efficiencies, the $\mathrm{Pb}$-glass converter afficiencies, and the probability of a gamma ray passing through the scintillator/veto in front of the detector.

A calculation of the converter efficiency has been completed using the standard EGS program which simulates the electron-gamma interaction in the converter. The simulation has been rull for the various energies of the $\pi$ 0 decay photons. Thie EGS program determines the converter efficiency from the probability that a gamma ray with a certain energy will convert in the converter and also gives the efficiency with the requirement that the resulting electron-positron shower produces a detectable charged particle in the multi-wire proportional chambers (MVVPC's). Figure 1 is a plot of these two efficiencies versus photon energy (the small variations in the curves are statistical in nature). The absolute measurement was made at $121 \mathrm{MeV}$ (square point on upper right side of plot).

Utilizing Monte Carlo results, comparisons were made to verify the values of various parameters used to analyze the data. For example, the distribution of certain measured parametars, such as eta, the opening angle between the two gamma rays in the $\pi^{\circ}$ decay, could be compared in detail with Monte Carls results. Various parameters were changed from their nominal values to give an idea of the accuracy of the experimental setup and the effect of these changes.

The case of the $40 \mathrm{MeV} \pi$ - beam scattered off a proton at a center-of-mass scattering angle of $0^{\circ}$ is considered here to give a picture of how the data were analyzed. Due to problems with the thin liquid hydrogen target, most of the E882 data were taken with a $\mathrm{CH} 2$ target. For these data, carbon target runs and no target runs were made so that the scattering contribution of the carbon and other backgrounds could be removed. The analysis was accomplished using a multistep process involving the histogram manipulation program HMN. First, histograms viere created for each experimental run. There were several runs with both $\mathrm{CH} 2$ and carbon targets. A histogram for an empty run was also needed which is shown in Fig. 2. Then, all of the runs for each target were summed into a single histogram. The empty run was subtracted from both the total $\mathrm{CH} 2$ and the total carbon after normalization. The 
normalization values come from the background in the empty run. The histogram after the subtraction is given in Fig. 3 for the $\mathrm{CH} 2$ arid in Fig. 4 for the carbon. At this point, the normalized net carbon was su'stracted from the net $\mathrm{CH} 2$. This normalization was calculated from the incident beam flux and the target thickness. in Fig. 5, a single histogram is given which contains data which are equivalent to the data obtained if a liquid hydrogen target were used.

The data obtained were compared to a Monte Carlo simulation using nominal and changed parameters. The Monte Carlo used was a modified version of PIANG that permits the direr! overlay of Monte Carlo plots and data plots. Histograms were created with the following parameter changes: (1) Beam Energy, (2) Beam Position in the $X$ and $Y$ direction relative to the target, (3) Target Position in the $Z$ direction relative to the pivot between the two arms of the spectrometer. A total of nine PIANG histograms were created for comparison in the $40 \mathrm{MeV}, 0^{\circ} \mathrm{cuse}$. The PIANG nominal histogram is given in Fig. 6 . It should be emphasized that the solid angle is calculated from PIANG for absolute measurements. The accuracy of this determination depends on how well the program predicts the reconstructed observables.

The results for E882 were reported at The Pion-Nucleon Symposium held at Bad Honnef, Germany on Sept. 9-13, 1991. The paper delivered there follows Figure 6 and aives the present cross section determinations.

\section{Converter Efficiency -vs- Photon Energy}

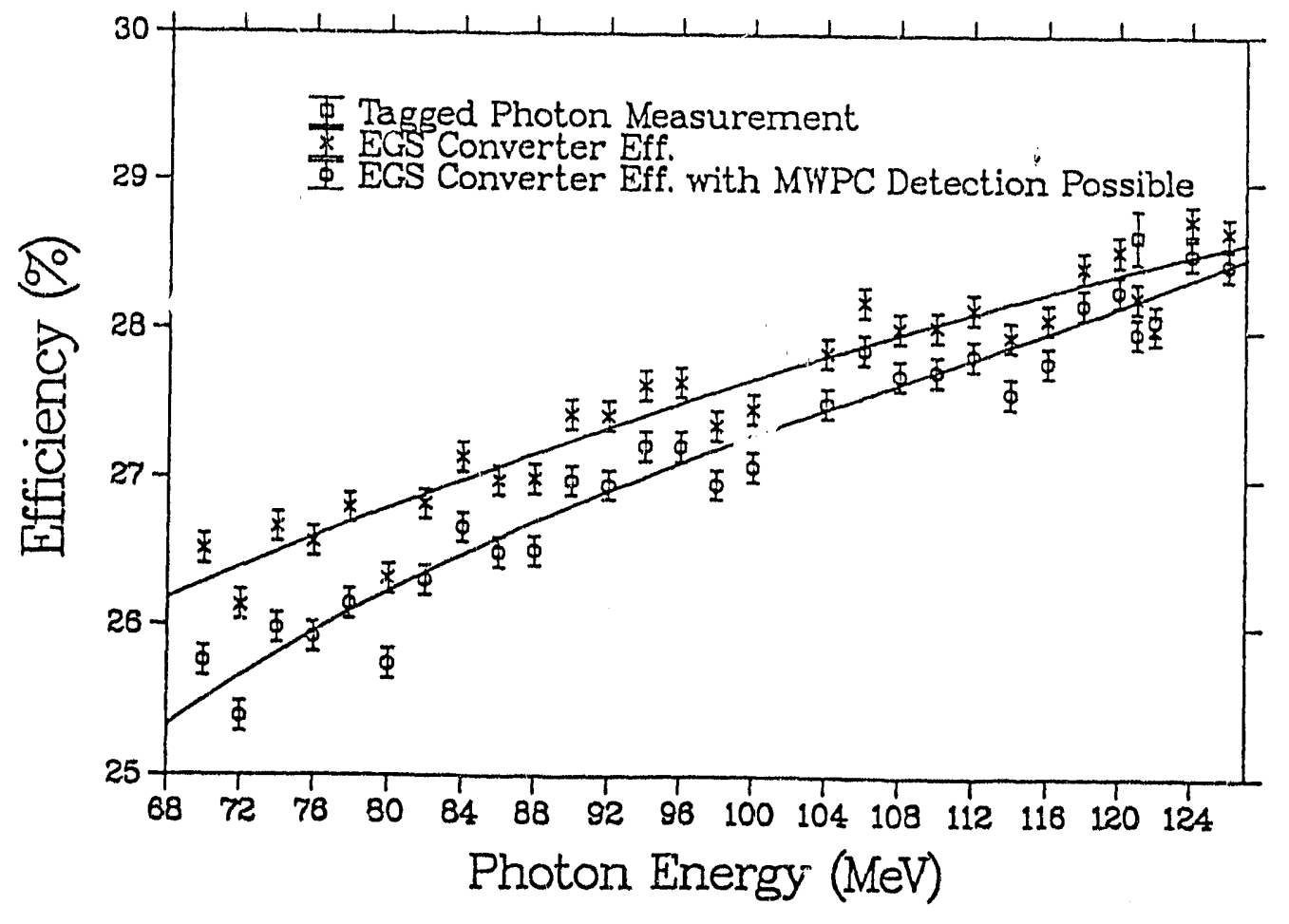

Figure 1 


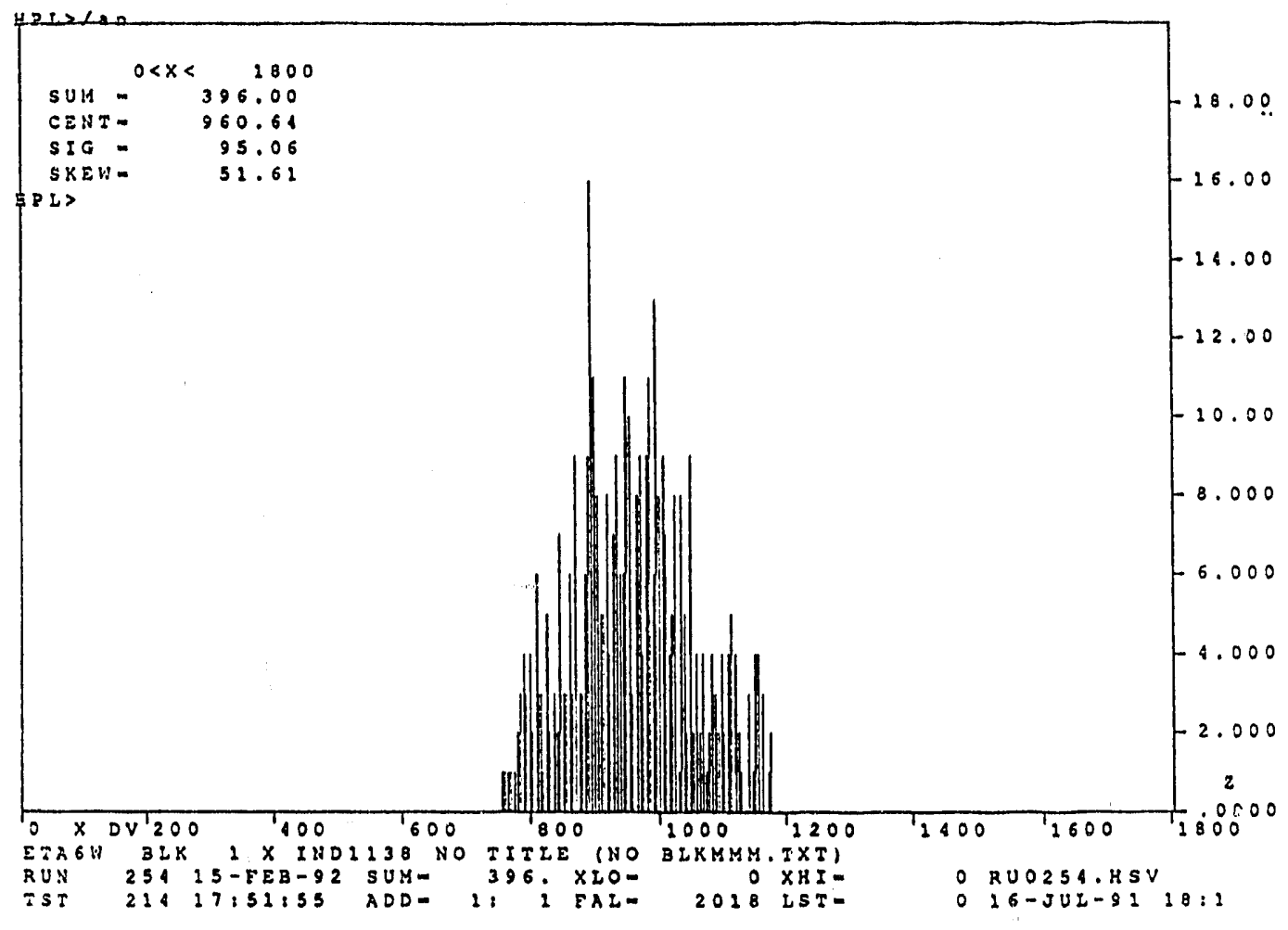

Figure 2

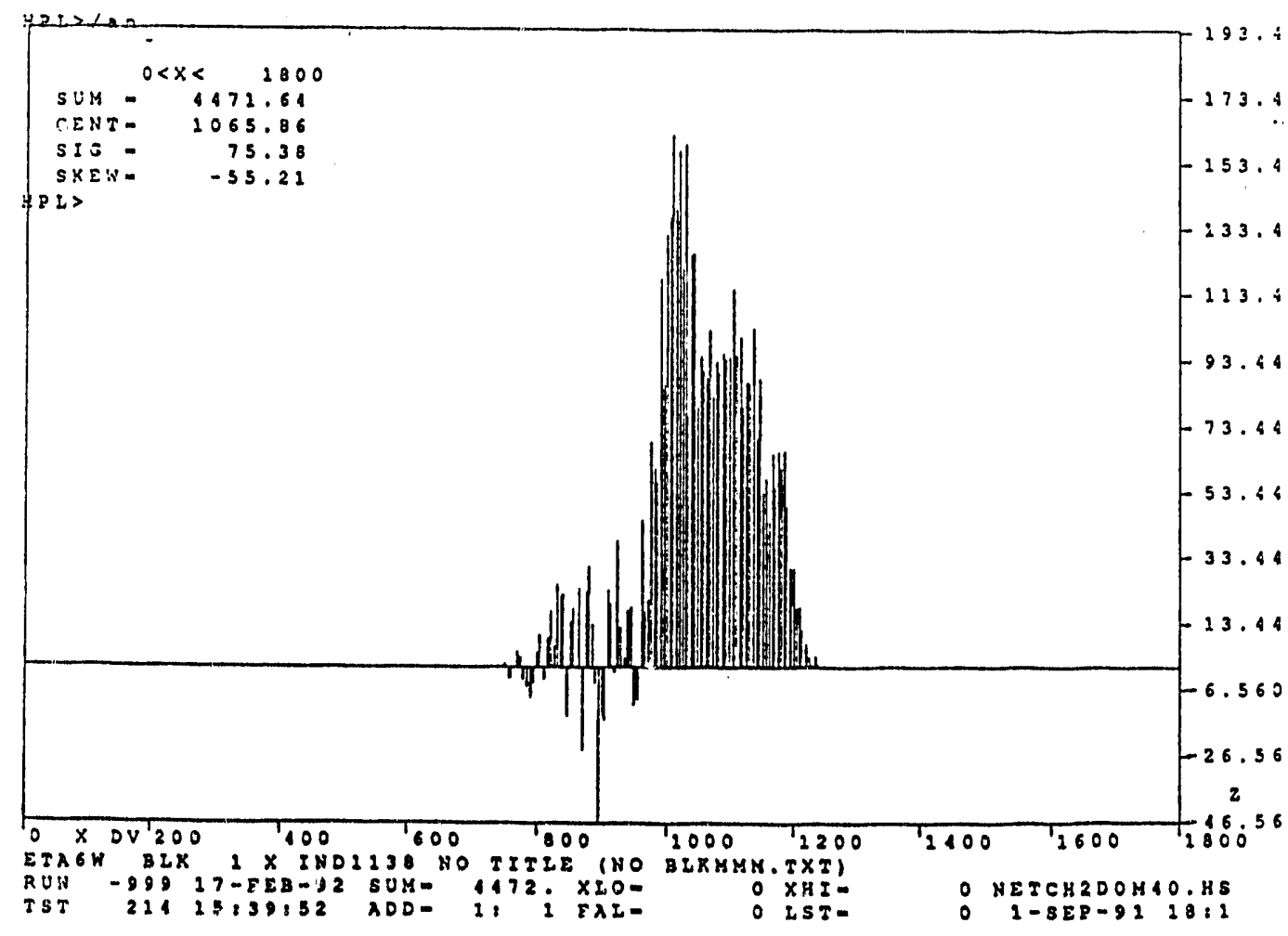

Figure 3 


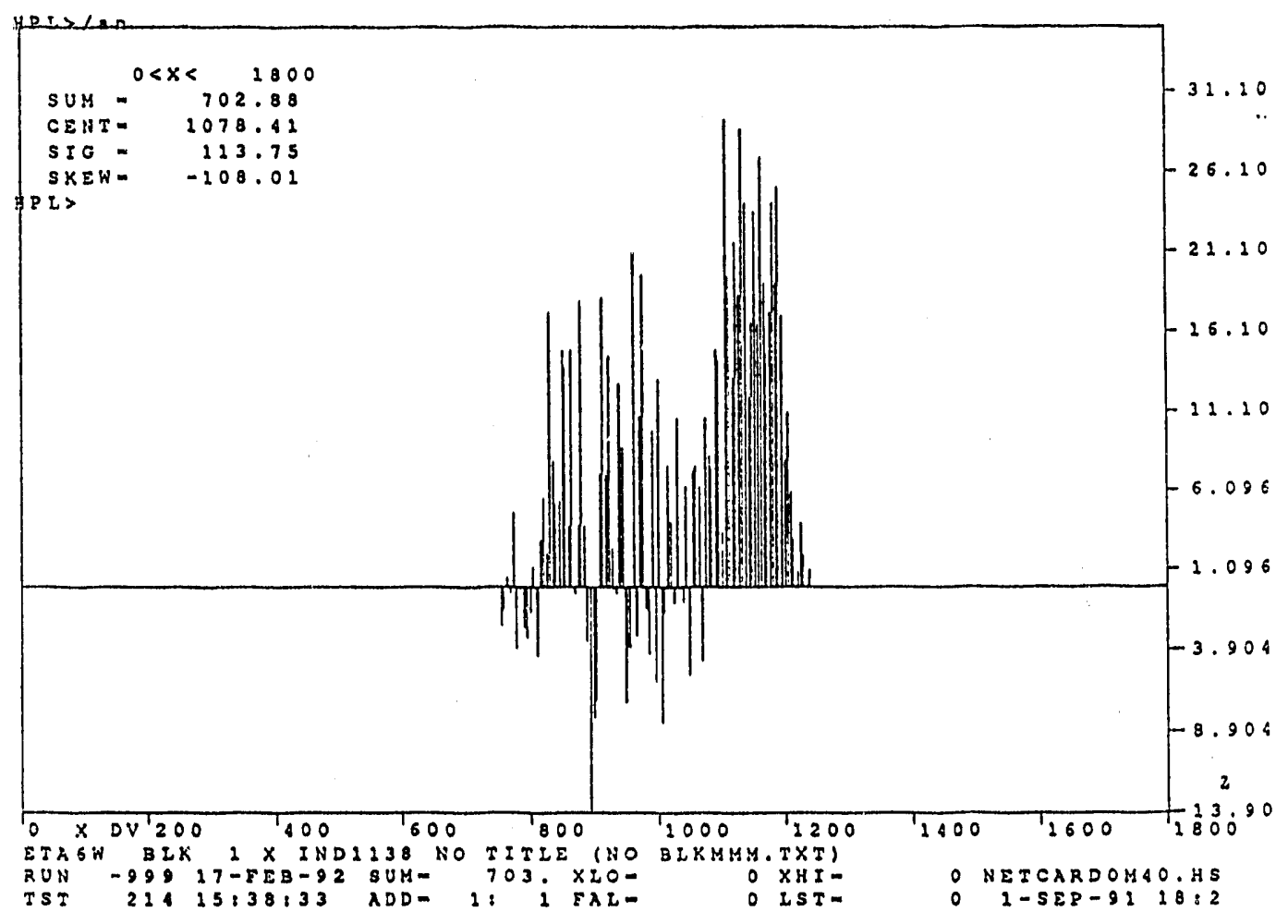

Figure 4

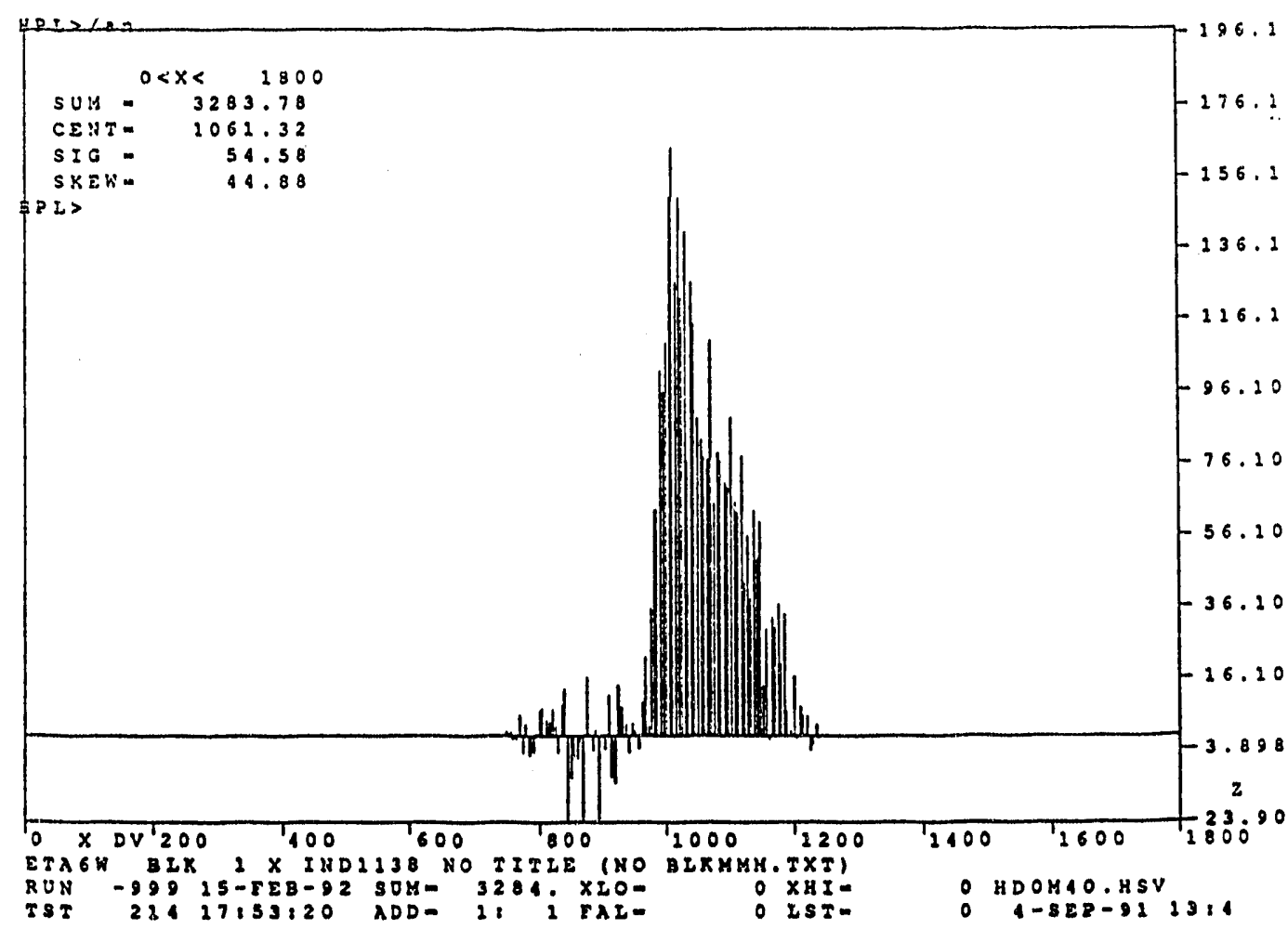

Figure 5 


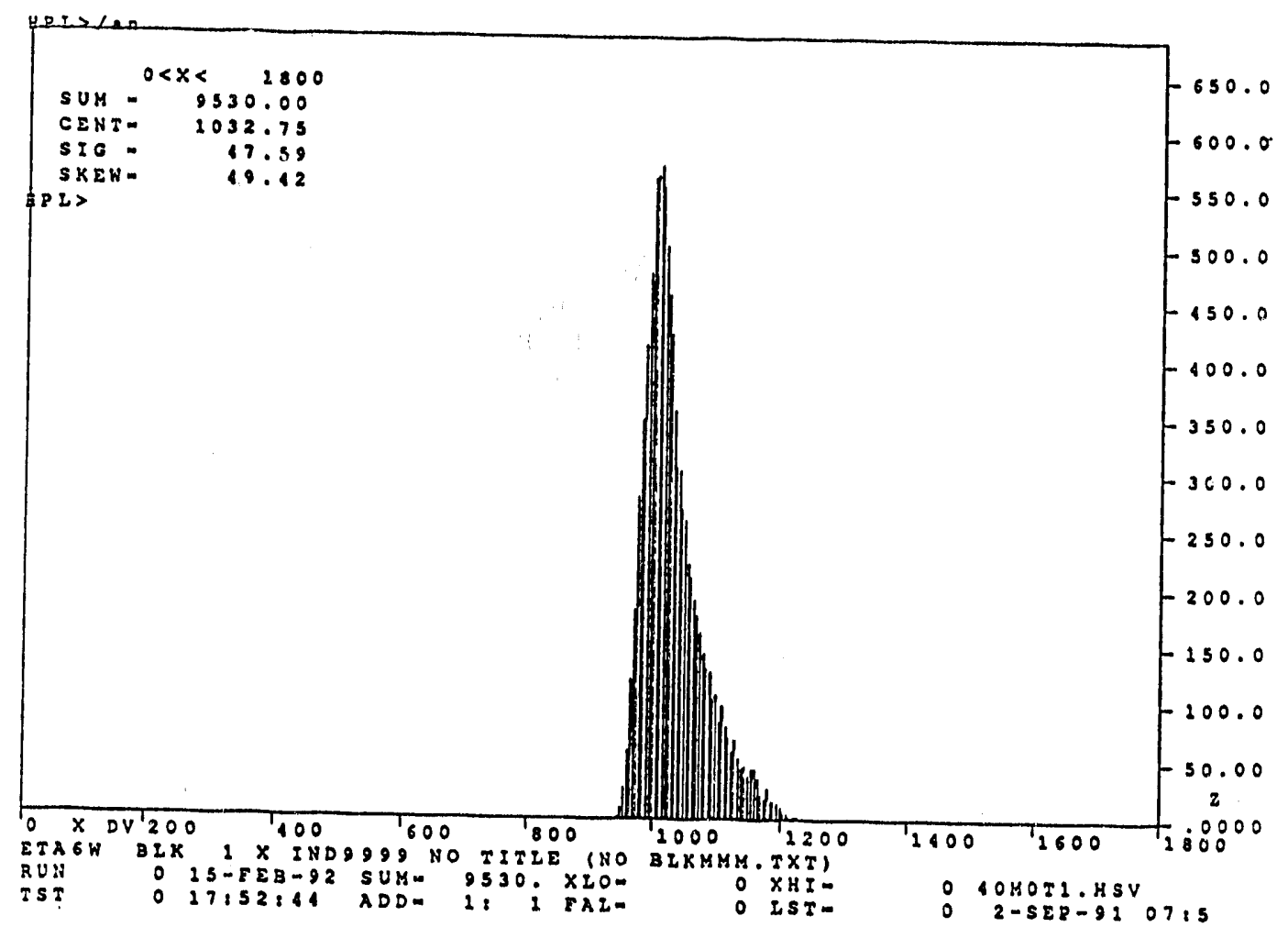

Figure 6 
FORWARD-AND BACKWARD-ANGLE DIFFERENTIAL CROSS SECTIONS

FOR $\pi \cdot p \rightarrow \pi \circ$ AT T $_{\pi}=10,20$, AND $40 \mathrm{MEV}$

M. E. Sadler, B. M. Brooks and L. D. Isenhower

Abilene Christian University, Abilene, TX 79699,

W. J. Briscoe

The George Washington University, Washington, DC 20052, and

J. D. Bowman, D. H. Fitzgerald and J. N. Knudson

Los Alamos National Laboratory, Los Alamos, NM 87545

\begin{abstract}
Results are given for measurements of the differential cross sections for $\pi \cdot p \rightarrow \pi \cdot n$ near 00 and 1800 at $T_{\pi}=10,20$, and $40 \mathrm{Mev}$, LAMPF Experiment 882 . The results are compared with the Karlsruhe and VPI partial wave analyses and the potential model of Siegel and Gibbs.

Differential cross sections were obtained for $\pi \cdot p \rightarrow \pi$ on using the LAMPF PIO Spectrometer1] to detect the $2 \gamma$ s from the decay of the $\pi^{\circ}$ at very low energies $\left(T_{\pi^{-}}=10,20\right.$, and $\left.40 \mathrm{Mev}\right)$. The two arms were placed symmetrically about the beam axis in order to measure near 00 and 1800. The acceptance extended approximately over the range $1 \geq \cos \theta \geq 0.85$ and $-0.85 \geq \cos$ $\theta \geq-1$ for the forward and backward measurements, respectively, where $\theta$ is the $\mathrm{cm}$ scattering angle of the $\pi^{\circ}$. The experiment was performed at LAMPF in the LEP channel with the inclusion of a crossed-field DC separator which dramatically reduced the electron and muon contamination of the pion beam. A liquid hydrogen target was used at forward angles at 10 and $20 \mathrm{MeV}$, but a backup $\mathrm{CH}_{2}$ target was used for the remainder of the experiment after the containment vessel imploded.
\end{abstract}

The PIO spectrometer consists of two arms with conversion planes and a Pb-glass calorimeter to measure both the coordinates and total energy of each $\gamma$ ray. Each arm has three conversion planes, each of which consists of 0.58 radiation lengths of $\mathrm{Pb}$-glass, three multiwire proportional chambers (MWPC's) and 3 mm of scintillator. The efficiency of a single 
conversion plane was measured, again using the $\pi-p \rightarrow \pi 0$ reaction but at $T_{\pi^{-}}=100 \mathrm{MeV}$ where the beam rate is higher. Two of the conversion planes in one arm were removed and the conjugate arm was used to tag $\gamma$ 's entering the converter. The result was $28.64 \pm 0.18 \%$ for a gamma energy of $121 \mathrm{MeV}$ (equal to half of the $\pi_{0}$ total energy). This value is in reasonable agreement with $28.23 \pm 0.05 \%$ obtained from a simulation using the EGS4 code2] after corrections for the distribution in incidence angle are taken into account. The simulation required a detectable charged particle to emerge from the converter. The EGS4 code was used to determine the energy dependence needed to extrapolate the measured result (which includes the scintillator and wire chamber efficiencies) to the energies for gammas detected in this experiment. The resulting efficiencies range from $26.1 \%$ at $72 \mathrm{MeV}$ to $27.1 \%$ at $89 \mathrm{MeV}$.

The scattering angle of the $\pi \circ$ is reconstructed from the measured energy and direction of the two gamma rays. Yields for three different angle bins were obtained in the analysis. These yields were obtained for two different fiducial areas of the wire chambers and two different cuts on the energy sharing parameter $X=\left(E_{1}-E_{2}\right) /\left(E_{1}+E_{2}\right)$, where $E_{1}, E_{2}$ are the individual gamma energies. The PIANG Monte-Carlo program was used to evaluate the geometrical acceptance for each angle bin, set of fiducial areas (corresponding to requiring the gamma ray to pass through a minimum of 6 and 8 radiation lengths of Pb-glass absorber), and energy sharing parameter $(|X| \leq 0.2$ and $|X| \leq 1.0)$. The four different results for a given angle bin scaled remarkably well with the four acceptance calculations.

Corrections were applied for conversions of gamma rays in the target, air, polyethylene plate (used to absorb slow charged particles) and veto scintillator. A sampling grid scincillator (0.73\% active area) was used to calibrate the pion beam. Muons and electrons were separated by pulse height at these low energies. The overall normalization uncertainties are estimated to be $\sim 8 \%$.

Forward-angle results are shown in Fig. 1. Predictions from the Karlsruhe-Helsinki3] and VPI4] phase shift analyses and the potential model of Siegel and Gibbs'] are shown for 


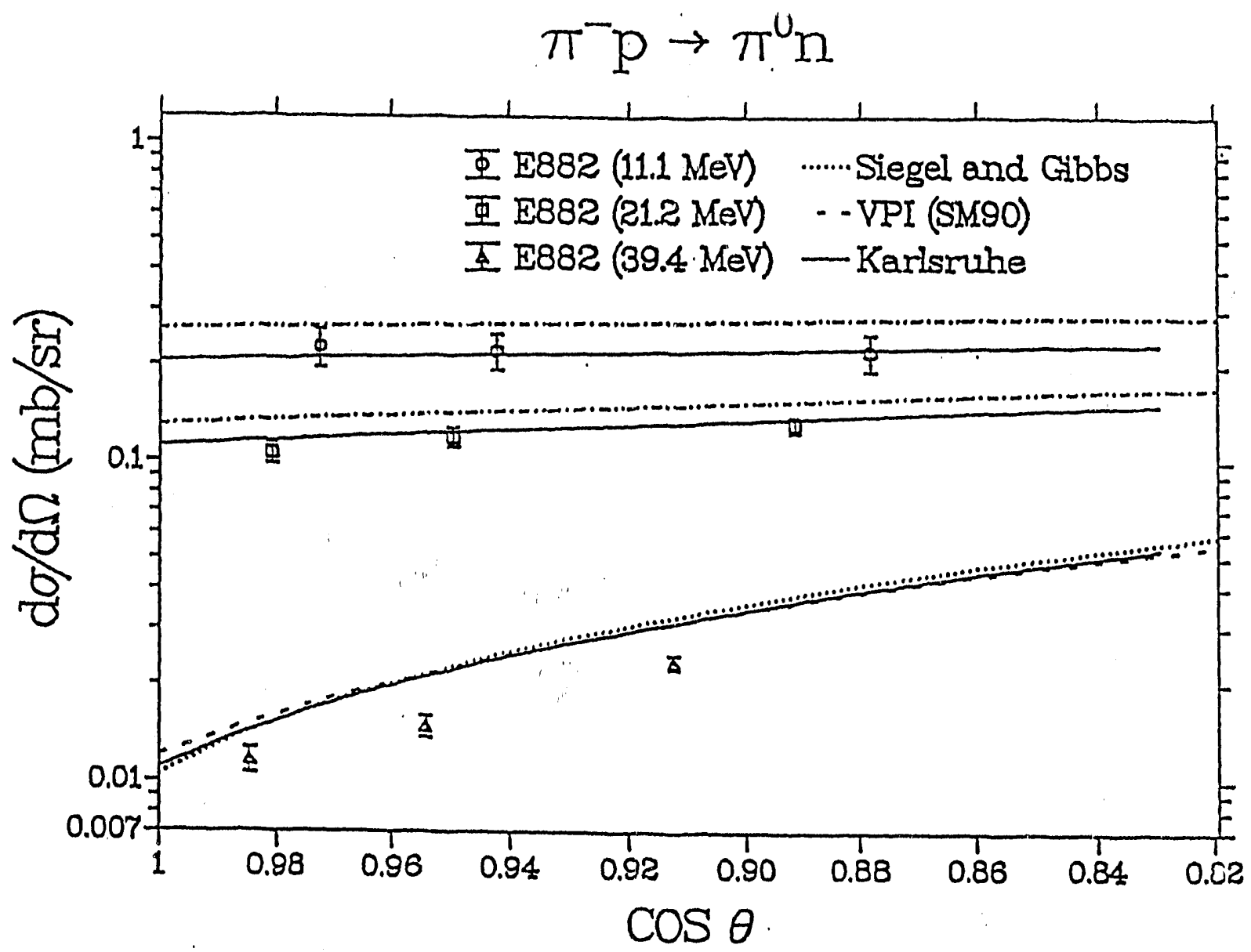

Fig. 1: Forward-angle differential cross sections. The errors are statistical only.

comparison. The data at $11.1 \mathrm{MeV}$ and $21.2 \mathrm{MeV}$ agree very well with the existing analyses, especially Ref. 3. The data at $39.4 \mathrm{MeV}$ are $15 \%$ below the consensus of the analyses. This difference is a consequence of the analyses from Ref. 3 and 4 fitting a previous measurement by our group 61 , which measured forward-angle cross sections in the region of the dip. The ostensible difference in the results is that backgrounds from scattering in air (nomalized only to pion flux) were deduced from carbon target runs (which are normalized to both pion flux and the ratio of carbon atoms in the targets) in the previous measurement. Background subtractions for both carbon and blank targets were performed in the present experiment. If only a carbon subtraction is performed for the present data at $39.4 \mathrm{MeV}$ where the cross sections are only 10 $20 \mu \mathrm{b} / \mathrm{sr}$, the present results overlap reasonably well with Ref. G.

In the backward direction, shown in Fig. 2, the data and the predictions agree reasonably well at $10.4 \mathrm{MeV}$ and $39.4 \mathrm{MeV}$ within the statistical errors. The predictions at $20.7 \mathrm{MeV}$ are $-20 \%$ 


$$
\pi^{-} p \rightarrow \pi^{0} \mathrm{n}
$$

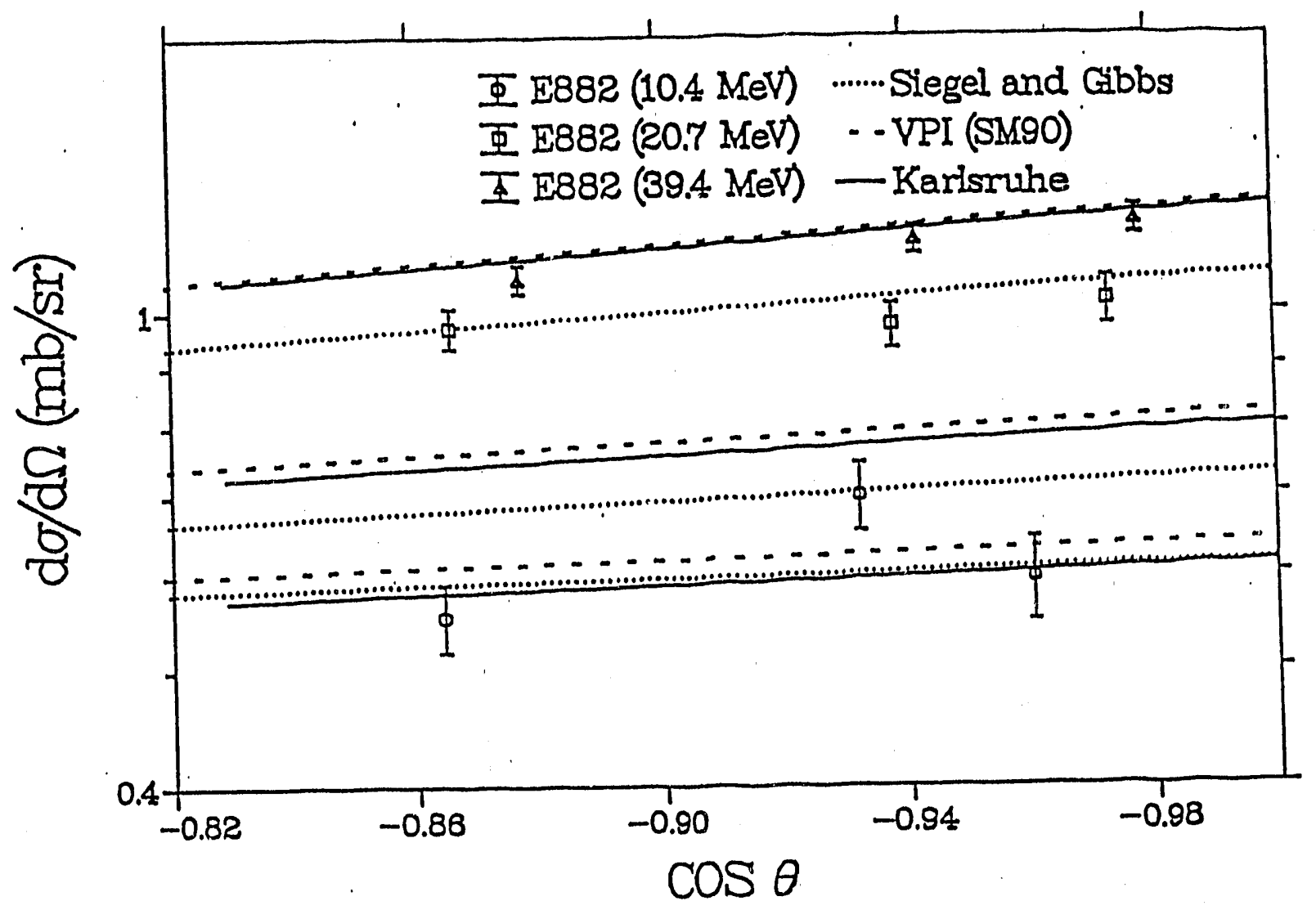

Fig. 2: Backward-angle differential cross sections.

lower than the data. This feature has persisted after two independent analyses. The data require a different energy dependence of the phase shifts for $s$ or $p$ waves in at least one of the isospin states, as discussed in Ref. 5].

References:

1] H. Baer, et al., Nucl. Inst. and Meth. 180, 445 (1981).

2] W. R. Nelson, H. Hirayama and D. W. O. Rogers, SLAC Report 265 (1985).

3] R. Koch and E. Pietarinen, Nucl. Phys. A336, 331 (1980).

R. Koch, Nucl. Phys. A448, 707 (1986).

G. Höhler, Landolt-Bömstein Vol. I/9b; Springer-Verlag (1983).

4] R. A. Andt, J. M. Ford and L. D. Roper, Phys. Rev. D32, 1085 (1985). The SM90 solution was obtained from the SAID program provided by $R$. A. Amdt.

5] P. B. Siegel and W. R. Gibbs, Phys. Rev. C33, 1407 (1980).

ఏ D. H. Fitzgerald, et al, Phys. Rev. C34, 619 (1980): 


\section{Experiment 849: Medium Energy Single Charge Exchange}

Experiment 849 was conducted in collaboration with W. J. Briscoe, C. Seftor, and N. J. Nicholas of George Washington University and J. Knudson of LAMPF. ACU participants were Sadler, Isenhower, I. Supek, M. Brooks, A. Donley, D. Lane, V. McVeigh, $L$. Towell and $R$. Towell. Differential cross sections for $\pi-p \rightarrow \pi$ on were measured near $0^{\circ}$ and $180^{\circ}$ at $P_{\pi}=427,471,509,547,586,625,657$ and $687 \mathrm{MeV} / \mathrm{c}$ in the $\mathrm{P}^{3}$ beam channel at LAMPF. The $\pi^{\circ}$ spectrometer was used to detect the decay $\gamma$ rays from the $\pi$.

Analysis of the forward-angle data was the subject of a GWU Master's thesis just completed by Nicholas and was reported on in the previous progress report for this grant. Analysis of the full data sample will be the Ph.D thesis of S.N. Dragic, another GWU student.

\section{Proposed Program with the New Neutral Meson Spectrometer}

The experience gained by this effort in using the existing $\pi$ Spectrometer to measure absolute cross sections for $\pi \cdot p \rightarrow \pi$ on has led to an active involvement in the development of the new Neutral Meson Spectrometer (NMS) at LAMPF. The objective is to utilize the same basic design as for the $\pi$ o Spectrometer, but to improve the energy resolution, vertex resolution of the conversion point of the $\gamma$ rays, data acquisition rate, solid angle, and versatility. A distinct advantage of the NMS over the existing spectrometer will be a better efficiency determination for the $\gamma$ conversion process and track reconstruction using the wire chambers, a necessity for any fundamental measurement that has to determine its own normalization such as those described in the preceding section.

Sadler has been active on the committee for the design and implementation of the NMS and has made res llar trips to LAMPF for the meetings of this committee. Two ACU students, Jason Phillips and Scott Garner assisted during the summer of 1990 in the construction of wire chambers for the NMS and the related tomography studies (described below). Keith Elmore and Tyson Browning spent the summer of 1991 at LAMPF assembling the tomography apparatus and developing software for the analysis. Since the principal investigators have spent most of these summers at Feimilab, St. Petersburg and CERN, these students have been supervised primarily by LAMPF personnel (primarily Jim Amann, Lee Atencio, Dick Boudrie, Chris Morris and Mark Whitton, all of MP-10). This arrangement has worked out well for both efforts in that increased summer research opportunities have been made available to ACU students and the students have made meaningful contributions to the NMS effort. 
Enough of the elements of the NMS are scheduled for delivery during the spring and summer of 1992 to start a developmental program with the device. These components include at least 60 of the 120 cesium-iodide (CsI) crystals for the calorimeter, enough BGO and cathode-strip-readout chambers for two conversion planes (one in each arm) and the accompanying electronics and cabling. The NMS committee agreed that two experiments, namely measurements of $\pi \cdot p \rightarrow \pi \circ n$ and $\pi \cdot d$ $\rightarrow \pi \circ n n$ with the NMS set up in the LAMPF $\mathrm{P}_{3}$ channel, were the most likely to yield interesting physics and still be suitable for development. These experiments were submitted as Exp. 1268, $\pi \cdot p \rightarrow \pi 0 n$ Cross Sections in the Region of the $\Delta$ Resonance, M. E. Sadler, spokesperson, and Exp. 1272, Pion Single Charge Exchange on the Deuteron ( $\pi d \rightarrow \pi \circ n)$ ): A Tune-up Experiment for the Neutral Meson Spectrometer, J. L. Matthews (Massachusetts Institute of Technology), and M. Whitton (Los Aiamos National Laboratory), spokespersons. Both experiments were approved with A priority by the LAMPF Program Advisory Committee. In particular, the committee stated:

We applaud the NMS collaboration's sooperative effort in organizing a plan for the near term NMS operation; the committee agrees in most aspects with its recommendations. We strongly encourage this group to continue in a cooperative planning mode. The availability of the NMS makes it timely for the existing $\pi^{\circ}$ spectrometer to be decommissioned, and we understand this is indeed planned following 1992 running.

In regards to Exp. 1268, the PAC statement consisted of the following:

It is proposed to measure $\pi$-p $\rightarrow \pi$ on differential cross sections in the resonance region using the NMS spectrometer. The physics motivation for this experiment is very solid. It is important to obtain more accurate data than are presently available to improve our knowledge of the $\pi \mathrm{N}$ phase shifts and to better determine the isobar splitting of the $\Delta$. However, at a time when the NMS is incomplete, this first phase of the experiment should be devoted exclusively to a study of its instrumental properties. Recommendation: As a necessary NMS development effort, approve for 125 hours at A priority.

The written proposal, included below, summarizes the present status of charge exchange measurements and emphasizes the need to develop the NMS as an absolute cletector, not as a device that relies on normalization to another process. The proposal as written requests 300 hours. This number was reduced to 125 hours for the development phase when presented to the FAC as a result of discussions in a NMS committee meeting the day before. 
Research Proposal

Clinton P. Anderson Meson Physics Facillty $\pi-p \rightarrow \pi$ on Cross Sections in the Region of the $\Delta$ Resonance

Spokesperson: M. E. Sadler

Participants:

L. D. Isenhower

J. R. Comfort

H. Crannell

W. J. Briscoe

J. Amann

R. Boudrie

J. Kriudson

C. Morris

J. O'Donnell

R. M. Whitton

J. Matthews

H. Park

J. Langenbrunner

G. Burleson

G. Kyle

M. Rawool-Sullivan

I. Supek
Abilene Christian University

Abilene, TX 79699

SADLER@ACUVAX

Abilene Christian University

Arizona State University

Catholic University of America

George Washington University

LAMPF

Massachusetts Institute of Technology

University of Minnesota

New Mexico State IJniversity

Plus other members of the NMS collaboration.

2 December 1991 


\section{Proposal Information}

Beam Area:

P3 Channel (Could also be done in the LEP chanriel, but NMS development is anticipated in $\mathrm{P}^{3}$ during Summer, 1992.)

Beam Requirements: $\quad \pi$ - Beam

Momenta: 229, 260, 302, 337 and $359 \mathrm{MeV} / \mathrm{c}$

(Kinetic Energies: 129, 156, 193, 225 and $246 \mathrm{MeV}$ )

Intensity: $\sim 5 \cdot 106 \pi / \mathrm{s}$

Duty factor: $10 \%$ preferred

Major LAMPF Apparatus: Neutral Meson Spectrometer (NMS)

Scheduling: $\quad$ Late Summer 1992

Hours Requested: $\quad 300$

\section{Abstract}

We propose to measure $\pi-p \rightarrow \pi \circ$ differential cross sections in the region of the $\Delta(1232)$ resonance. Elements of the new Neutral Meson Spectrometer (NMS) will be used to measure the two $\gamma$ rays from the $\pi^{\circ}$ decay, eliminating the difficulty of determining the efficiency of neutron counters. The design energy resolution of the NMS is not needed for this reaction, making it a good candidate for obtaining useful physics before the device is fully commissioned.

The motivation for measuring these cross sections in the region of the $\Delta$ is summarized below.

- To check existing data which do not agree well with partial wave analyses (PWA's) at the back angles near the $\Delta$.

- To provide accuratu data for input to charge-dependent PWA's and to investigate charge splitting of the $\Delta$.

- To complement existing and future elastic measurements that should help clarify discrepancies at lower energy through analyticity constraints of the PWA's. 


\section{Introduction}

The simple spin and isospin structure of the pion-nucleon system makes it a unique source of information on the hadronic interaction. Assuming isospin invariance, the scattering amplitudes for the three observable reactions are functions of only two independent isospin amplitudes, $F_{3 / 2}$ and $F_{1 / 2}$. These relations are:

$$
\begin{aligned}
& F\left(\pi^{+} p \rightarrow \pi^{+} p\right) \equiv F^{+}=F^{3 / 2} \\
& F\left(\pi^{-} p \rightarrow \pi^{-} p\right) \equiv F^{-}=\frac{1}{3} F^{3 / 2}+\frac{2}{3} F^{1 / 2} \\
& F\left(\pi^{-} p \rightarrow \pi^{0} n\right) \equiv F^{\circ}=\frac{\sqrt{2}}{3}\left(F^{3 / 2}-F^{1 / 2}\right)
\end{aligned}
$$

Thus, the amplitudes describing the three observable reactions are related by

$$
\sqrt{2} F^{\circ}=F^{+}-F^{-}
$$

Each of these amplitudes can be written in the form

$$
F(k, \theta)=G(k, \theta)+i H(k, \theta) \hat{n} \cdot \vec{\sigma}
$$

where $G$ and $H$ are the non-spin-flip and spin.flip amplitudes, respectively, $\widehat{n}$ is the unit normal to the scattering plane and $\vec{\sigma}$ is the Pauli spinor for the nucleon. The dependence of the amplitudes on the $\mathrm{cm}$ momentum, $k$, and scattering angle, $\theta$, is indicated explicitly. Thus, assuming isospin invariance, only four (complex) scattering amplitudes are necessary to describe all pion-nucleon interactions at a given incident momentum and scattering angle. This gives seven independent real quantities since an overall phase is unobservable. The amplitudes must be corrected for Coulomb effects for equation (2) to be valid.

The differential cross sections are the squares of the amplitudes which, for an unpolarized target, are given by

$$
\frac{d \sigma}{d \Omega}(k, \theta)=|G(k, \theta)|^{2}+|H(k, \theta)|^{2}
$$

and the amplitudes can be expanded into partial waves (after Coulomb corrections): 


$$
\begin{aligned}
& G(k, \theta)=\frac{1}{k} \sum_{l=0}^{\infty}\left\{(l+1) T_{\ell+}(k)+l T_{\ell-}(k)\right\} P_{\ell}(\cos \theta) \\
& H(k, \theta)=\frac{1}{k_{l=1}} \sum_{l=1}^{\infty}\left\{T_{\ell+}(k)-T_{\ell-}(k)\right\} \sin \theta P_{\ell}^{\prime}(\cos \theta)
\end{aligned}
$$

where $T_{\ell} \pm$ are the dimensionless partial wave amplitudes for $j=\ell \pm 1 / 2$ and $P_{\ell}(\cos \theta)$ are the Legendre polynomials. Equation (1) can be used to write the partial wave amplitudes for a given reaction in terms of its isospin-3/2 and isospin-1/2 components. For example, for charge exchange scattering we have

$$
T_{\ell \pm}^{0}=\frac{\sqrt{2}}{3}\left(T_{\ell \pm}^{3 / 2}-T_{\ell \pm}^{1 / 2}\right)
$$

The partial wave amplitudes for given isospin, $l, \ell$, and $j=\ell \pm 1 / 2$ can be written in terms of the real variables $\delta_{\ell_{ \pm}}^{l}$ (phase shifts) and $\eta_{\ell_{ \pm}}$(absorption parameters) as:

$$
T_{\ell_{ \pm}}^{\prime}=\frac{\eta \ell_{ \pm} e^{2 i \delta \ell_{ \pm}-1}}{2 i}
$$

S- and $P$-waves strongly dominate pion-nucleon scattering up to the $\Delta(1232)$ resonance, where $\frac{3}{\delta_{\frac{3}{2}}^{2}} \equiv \delta\left(P_{33}\right)=90^{\circ}$. Ignoring higher partial waves, there are only six phase shifts and six absorption parameters to be determined at a given value of $k$ (using the $l_{21,2 j}$ notation, the partial waves are $S_{11}, S_{31}, P_{11}, F_{31}, P_{13}$ and $P_{33}$ ). Three Legendre coefficients can be obtained from measurements of the differential cross sections from each reaction (total of nine parameters). Six additional parameters can be obtained from polarization measuremenis. Still more information can be obtained from measurements in the Coulomb interference region and from total cross sections. The phase shifts are strongly over determined in this region, allowing the possibility of a reliable treatment of electromagnetic effects and a search for isospin breaking. These investigations are only as good as the data on which they are based.

\section{Survey of Existing $\pi \mathrm{N}$ Charge Exchange Data and Partial Wave Analyses in the Reglon of the $\Delta$}

The most extensive set of differential cross sections for $\pi \mathrm{N}$ charge exchange in the region of the $\triangle$ are the CERN data of Jenefsky, et al., [Je77], who made measurements at eight momenta from 229 to $359 \mathrm{MeV} / \mathrm{C}\left(128 \mathrm{MeV} \leq \mathrm{T}_{\pi} \leq 246 \mathrm{MeV}\right.$ ) in 
the angular range $-0.98 \leq \cos \theta_{\pi^{\circ}} \leq 0.65$. Total (statistical plus systematic) errors of 6 $10 \%$ are reported. Neutrons only were detected, identified by time-of-flight (TOF). These data are plotted in Fig. 1-4 at $P_{\pi}=229,293,302$, and $359 \mathrm{MeV} / \mathrm{c}$. Included in each plot are comparisons to the Karisruhe-Helsinki [Hö79,K080] and Virginia Polytechnic Institute [Ar85] partial wave analyses. There is little difference between the predictions from these two solutions throughout the energy region. Tr: $\theta$ analyses agree very well with the data below the $\Delta$ (see Fig. 1, also true at $260 \mathrm{MeV} / \mathrm{c}$ ), but are consistently lower (by 1-3 times the quoted errors) at the back angles on and above rescnance.

Note the almost perfect $\cos ^{2} \theta$ shape of the data and PWA's in Fig. 2, which is the signature of the P-wave resonance. The UCLA data of Comiso, et al., [Co75] at $2.95 \mathrm{MeV} / \mathrm{c}$ are included for comparison. These data were taken with a neutrongamma coincidence requirement. A total of five angles (nominally $-0.8 \leq \cos \theta_{\pi^{\circ}} \leq 0.5$ ) at five beam momenta from 239 to $375 \mathrm{MeV} / \mathrm{c}$ were measured at the LBL synchrocyclotron. The total errors are $7-11 \%$ for most of the data and they agree well with the analyses to this accuracy. Similar data by the same group [Be72] also exist above resonance at 317,452 and $491 \mathrm{MeV} / \mathrm{c}$.

The forward-angle cross sections of Bayer, et al., [Ba76] are included in Fig. 3 and 4. The cornplete data set consists of measurements at $\theta_{\pi^{\circ}}=0^{\circ}$ at nine momenta from 244 to $388 \mathrm{MeV} / \mathrm{c}$. The experiment utilized a Nal(TI) photon detector to measure both the energy and TOF of single photons, using a magnet to deflect the beam after passi.ig through a liquid hydrogen target. The quoted errors are $6-8 \%$.

Also shown in Fig. 4 are data of Hauser, et al., [Ha71], which were taken at the Princeton-Penn accelerator to provide new evidence for the $P_{11}$ resonance which had just been reported. Excitation functions were measured with neutron detectors at fixed lab angles. The error bars shown are only statistical since the systematic errors are not specified, but the neutron detector efficiencies are "as large as 30\%" for the lower neutron energies (forward $\theta_{\pi}$ ) but estimated to be less than $10 \%$ for most of the data.

Finally, the total charge exchange cross section has been measured by Bugg, et al., [Bu71], to an accuracy of $1 \%$ between 90 and $290 \mathrm{MeV}$. The results are shown in Fig. 5. The technique used was to surrouna a liquid hydrogen target with a box of scintillation counters. The number of incident pions interacting to produce a neutral final state (no signal in the surrounding counters) was recorded. Corrections were applied for conversion of either the outgoing gamma rays or neutrons to charged particles $(1-3 \%)$ and neutrals from either $\pi \cdot p \rightarrow m$ or $\pi \cdot p \rightarrow \pi 0 \pi$ on $(1-2 \%)$. The small errors provide a mechanism to normalize other measurements of differential cross sections if enough of the angular distribution is sampled to obtain a reliable integrated cross section. Given the small error bars, the agreement with the PWA's in Fig. 5 is not good. The VPI and Karlsruhe analyses have a $\chi^{2}$ per data point of 18 and 24 , respectively, when compared with the data. 
Summarizing, the differential cross sections throughout the region of the $\Delta$ are larger than $1 \mathrm{mb} / \mathrm{sr}$ at all angles. Typical errors quoted by the groups are $6-10 \%$ and are largest at the back angles. A systematic measurement by one group of a complete angular distribution from near $0^{\circ}$ to near $180^{\circ}$ does not exist at any momentum. All data for angular distributions involve neutron counters. Characteristics of neutron counters are likely to change over time due to effects of crazing or optical coupling of the scintillator, or to threshold instabilities, monitoring and reproducibility. The next section discusses the use of the NMS to measure these cross sections.

\section{The Neutral Meson Spectrometer as an Absolute Detector}

A drawing of the NMS showing the conversion planes, calorimeter and support system is shown in Fig. 6 . Two distinct advantages that the NMS will offer compared to the detection techniques summarized above are 1) measurement of the $\pi^{\circ} \rightarrow 2 \gamma$ decay eliminates the need for neutron counters and 2) complete angular coverage $(-1 \leq$ $\cos \theta_{\pi^{\circ}} \leq 1$ ) is possible since the $2 \gamma^{\prime}$ s are always experimentally accessible.

The present $\pi^{\circ}$ spectrometer has been commonly used as a relative detector for most of its career, with the "known" cross sections from $\pi \cdot p \rightarrow \pi$ on used to normalize the data for absolute cross sections. Two published exceptions, both measurements of the differential cross section for $\pi \cdot p \rightarrow \pi^{\circ}$ n with the spectrometer at $0^{\circ}$, are the data of Gaille, et al., [Ga84] at $522 \mathrm{MeV} / \mathrm{c}$ and Fitzgerald, et al., [Fi86] at seven momenta from 100 to $147 \mathrm{MeV} / \mathrm{c}$ (22 to $63 \mathrm{MeV}$ ). Gaille, et al., obtained charge exchange data as one of the calibration procedures for the geometry used in their pion beta decay experiment [McF83] and quote a result of $4.32 \pm 0.11(2.6 \% !) \mathrm{mb} / \mathrm{sr}$. The wire chambers of the $\pi^{\circ}$ spectrometer were not used so uncertainties associated with reconstructing tracks were eliminated. A summed-energy signal for the $\pi^{\circ}$ was obtained with a resolution of $15 \%$, which was adequate for separating the background from $\pi-p \rightarrow \pi \circ \pi$ on. The NMS will have much better energy resolution due to the CsI (instead of $\mathrm{Pb}$-glass) used for the calorimeter.

Fitzgerald, et al., were much more conservative in their absolute error estimates, reporting an overall normalization uncertainty of $7.8 \%$. It is worthwhile to inspect the formula used by [Fi86] to calculate the differential cross section, since the same procedure will be used for absolute measurements with the NMS:

$$
\frac{d \sigma}{d \Omega}=\frac{Y J}{N\left(\pi^{\circ}\right) N_{H} \Omega\left(\pi^{0}\right) \varepsilon\left(\pi^{0}\right) \varepsilon_{W} f_{a b s} F_{\gamma} \tau_{L}}
$$

where $Y$ ' is yield, $J$ is the Jacobian of the transformation of the cross section from the lab to the c.m. frame, $N\left(\pi^{-}\right)$is the number of beam particles, $N_{H}$ is the areal density of hydrogen in the target, $\Omega\left(\pi^{\circ}\right)$ is the laboratory solid angle acceptance of the 
spectrometer for the two $\gamma$ rays, $\varepsilon\left(\pi^{\circ}\right)$ is the $\pi^{\circ}$ detection efficiency (the probability that both of the gamma rays will convert in one of three converters in each arm), $\varepsilon w$ is the overall wire chamber efficiency for detecting the charged particles emerging from the converter (including the track reconstruction), $f_{a b s}$ is the fraction of photons that make it to the spectrometer without first converting in the target, air, or veto counters, $F_{\gamma}$ is the $\pi^{\circ} \rightarrow \gamma$ branching ratio $(0.98802)$ and $\tau_{L}$ is the experimental livetime.

The only quantities in equation (8) that are peculiar to detecting neutral mesons are $\Omega(\pi 0), \varepsilon\left(\pi^{\circ}\right), \varepsilon_{W}$ and $f_{a b s}$. Everything else is determined by siandard techniques. The statistical uncertainties in the yields can easily be determined to $1 \%$ (see the rate estimates in the next section). Beam normalizations with uncertainties of a few percent have been obtained in other LAMPF experiments. For example, [Ga84] report $1 \%$ and [Sa87] obtained normalizations near $2 \%$ for most of the twenty beams in measurements of $\pi^{ \pm p} \rightarrow \pi^{ \pm p}$ differential cross sections from 378 to $687 \mathrm{MeV} / \mathrm{c}$ at LAMPF. Hydrogen target lengths can be determined to $1 \%$ if proper care is taken [Sa87]. Uncertainties in the livetime can be kept small by limiting the beam so that $\tau_{L}>$ $90 \%$.

$\Omega(\pi \circ)$ must be obtained from a Monte-Carlo calculation which incorporates the geometry of the beam, target and spectrometer. Consistency checks can be made using different cuts on energy sharing between the two arms and defining different fiducial areas in the wire chambers which follow the converters to detect the shower. A purely geometrical calculation has been performed by the PIANG program for the PIO spectrometer, but greater accuracy would be achieved if the physics of the shower propagation in the converters and wire chambers were included. Such a computer program is under development for the NMS [Par91] and is presently being used to determine the expected spatial resolution of gamma conversion points.

The overall $\pi^{\circ}$ detection efficiency, $\varepsilon(\pi \circ)$, for the PIO spectrometer is given by the expression

$$
\varepsilon(\pi 0)=\left[1-\left(1-\varepsilon_{c}\right)^{3}\right]^{2},
$$

where $\varepsilon_{c}$ is the efficiency for a single converter to produce a detectable charged particle. The cubic power in equation (9) follows from the three conversion planes per arm for the PIO spectrometer and will be replaced by a quartic for the four conversion planes per arm that are eventually planned for the NMS. $\varepsilon_{\mathrm{c}}$ depends on the gamma energy and was nominally $0.280 \pm 0.016(5.6 \%)$ in [Fi86]. This uncertainty can be reduced easily by a systematic study of the NMS converter efficiencies using tagged photon measurements with a fully assembled converter system to measure the $\varepsilon_{c} E_{W}$ product. Such measurements are planned as part of NMS development. Careful modelling of the electron-gamma showers using the Stanford EGS code or GEANT will be used to check the measurements. The modelling will take into account such effects as a conversion taking place but producing no charged particles in the region 
behind the converter (particularly significant for low yenergies) and occurrences of charged particles exiting backward into veto scintillators. This modelling is presently underway [Par91].

The quantity fabs, like $\varepsilon(\pi \circ)$, must be modelled using a shower code which includes the geometry of the experiment.

Another advantage that is derived from detecting the $\pi \circ \rightarrow \gamma \gamma$ decay is that a large angular interval is accepted for a given position of the spectrometer. For the $\gamma$ pairs accepted by a given geometry, the scattering angle is reconstructed from

$$
\cos \theta_{\pi^{\circ}}^{L}=\frac{E_{1} \cos \theta_{1}+E_{2} \cos \theta_{2}}{\left(E_{1}^{2}+E_{2}^{2}+2 E_{1} E_{2} \cos \eta\right)^{\frac{1}{2}}}
$$

where $\theta_{\pi^{\circ}}^{L}$ is the laboratory angle of the scattered $\pi^{\circ}, E_{1}, \theta_{1}\left(E_{2}, \theta_{2}\right)$ are the laboratory energy and angle, respectively, for the first (second) gamma ray and $\eta$ is the opening angle between the gamma rays. The NMS will subtend a lab angle of more than $40^{\circ}$ if it is positioned one meter from the target in the configuration shown in Fig. 6 . For a beam momentum of $302 \mathrm{MeV} / \mathrm{c}$ (see Table 2), a central angle of $20^{\circ}$ will measure a cm interval of $1.0 \leq \cos \theta_{\pi^{\circ}} \leq 0.63,80^{\circ}$ corresponds to $0.27 \leq \cos \theta_{\pi^{\circ}} \leq-0.43$, and $160^{\circ}$ to $-0.86 \leq \cos \theta_{\pi^{\circ}} \leq-1.0$.

The determination of $\theta_{\pi^{\circ}}^{L}$ from equation (10) requires uniform energy calibration of the Csl crystals in the calorimeter and good position resolution of the gamma rays. One reason that [Ga84] reported such a low uncertainty is that the wire chambers of the PIO spectrometer were not used to perform an angle binning using equation (10). The shape of the angular distribution was taken from the VPI PWA and folded into the geometrical acceptance. The Csl crystals (total of 120 in both arms) will provide position resolution for the NMS of $\sim 3 \mathrm{~cm}$ even without the use of wire chambers. By not requiring converter information in the trigger for events to be written to tape in the experiment, the calorimeter information can be used to determine $\varepsilon_{W}$ in equation (8).

\section{The Need for New Charge Exchange Data}

The differential-cross-section data for $\pi \pm p \rightarrow \pi \pm p$ and $\pi-p \rightarrow \pi \circ$ scattering are surprisingly sparse in the region of the $\Delta$. Partial-wave analysts essentially rely upon the measurements of Bussey, et al., [Bu73] who measured $\pi \pm p \rightarrow \pi \pm p$ elastic scattering with an accuracy of typically $2-5 \%$ in the range $180 \leq P_{\pi} \leq 408 \mathrm{MeV} / \mathrm{c}$. This data set replaced a collection of previous data which had uncertainties of $5-10 \%$, had been taken over a limited angular range, existed at only a few energies, and were sometimes in disagreement with energy-dependent partial wave analyses. At least one of these criticisms applies to each of the presently available data sets for $\pi-p \rightarrow$ $\pi \circ$ cross sections summarized in Sec. II. 
The $\pi \mathrm{N}$ system is too fundamental to allow one experiment to be the sole source of information over the range of the first resonance. Recently, a new measurement of $\pi \pm p \rightarrow \pi \pm p$ differential cross section sections has been approved at TRIUMF [Pav91] to check the results of Bussey, et al. A primary motivation for the new measurements is the inconsistency between the recent low-energy Colorado/TRIUMF measurements of Brack, et al. [Br86, Br88, Br90], with previously accepted results [Be76 and Bu73]. The Colorado/TRIUMF group measured $\pi \pm p \rightarrow \pi \pm p$ differential cross sections using three different methods from 96 to $241 \mathrm{MeV} / \mathrm{c}$ (30 to $139 \mathrm{MeV}$ ) with normalization errors of $2 \%$. Besides being in variance with most of the previous data (and the PWA's which include these data), the data are attacked by partial-wave analysts as being inconsistent with data at higher energy using constraints imposed by analyticity (dispersion relations)[Hö90 and Bu91]. Recently, D. V. Bugg remarked at the IVth $\pi \mathrm{N}$ Symposium in Bad Honnef, Germany, see [Pav91], that if the Brack data were correct then "all other $\pi \mathrm{N}$ data had to be thrown out" (meaning, presumably, the data of Bussey, et al., of which he was a coauthor). In addressing this issue, Höhler and Stahov [Hö90] state:

How can one resolve these discrepancies? One way, of course, is to repeat the $\pi \pm p$ differential and integral cross section measurements, but we think that it would be more useful to measure other quantities: to improve the accuracy of the charge-exchange cross sections and to measure analyzing powers $A_{N}=P$ for all three reactions.

(The emphasis is ours.) The discussion refers to the low-energy situation at 45 and 67 Mev, below the range of the present proposal. The NMS will be well-suited for this measurement also when it is commissioned and moved to the LEP channel. First, it is needed to demonstrate that the device can produce results of $5 \%$ overall accuracy in a region where the cross sections are large, the subject of the present proposal. It should be noted also that a proposal for a measurement of the analyzing powers hias already been approved at LAMPF[C089].

In order to make use of the relationships between the scattering amplitudes for the three observable $\pi \mathrm{N}$ reactions (see Sec. I), most PWA's assume isospin invariance after applying Coulomb corrections. It is obvious that isospin symmetry should be destroyed in the region of the $\Delta$ due to differences in masses and widths between the $\Delta^{++}$and $\Delta^{\circ}$. The $\pi \pm p$ and $\pi \pm d$ total cross section measurements of Pedroni, et al., [Pe78] yielded results of $\Delta M=M^{\circ}-M_{++}=2.7 \pm 0.3 \mathrm{MeV}$ and $\Delta \Gamma=\Gamma^{\circ}-$ $\Gamma^{++}=6.6 \pm 1.0 \mathrm{MeV}$. Abaev[Ab89] has allowed different $P_{33}$ phases for $\pi+p$ and $\pi$-p scattering in his partial wave analysis for $W(=\sqrt{s})$ from 1.2 to $1.5 \mathrm{GeV}\left(P_{\pi}\right.$ from 250 to $700 \mathrm{MeV} / \mathrm{c})$. His results for the difference, $\Delta \delta$, in these phases are shown in Fig. 7. The line at $\Delta \delta=0$ represents a relativistic Breit-Wigner fit to the 1980 Karlsruhe 
analysis [Hö79,K080], which assumes isospin invariance. The +'s are the actual differences between the fit and the Karlsruhe analysis. The solid (open) circles are the differences between the fit and the SPNPI analysis for $\pi+p(\pi-p)$ scattering. Abaev reported preliminary values of $\Delta M=2.2 \pm 0.6 \mathrm{MeV}$ and $\Delta \Gamma=4.6 \pm 1.3 \mathrm{MeV}$. It is interesting that the SPNPI analysis shows a systematic difference in the $P_{33}$ phases for the $\pi+p$ and $\pi-p$ analyses even at the upper end of the mass range. Note also that the error bars are somewhat larger for the $\pi$-p analyses (open circles), due primarily to the larger uncertainties in the cross sections for $\pi \cdot p \rightarrow \pi$ on.

\section{Beam Time Estimates}

It is proposed to measure differential cross sections for $\pi^{-p} p \rightarrow \pi$ on at laboratory angles of $20^{\circ}, 80^{\circ}$ and $160^{\circ}$ at five momenta near the $\Delta$ resonance $\left(P_{\pi^{-}}=229,260\right.$, $302,337$ and $358 \mathrm{MeV} / \mathrm{c})$. Kinematics and rate estimates are shown for all five beams in Tables 1-3. A maximum beam rate of $5.106 \pi / \mathrm{s}$ is assumed in order to monitor the beam reliably. A $2.5 \mathrm{~cm} \mathrm{CH}$ target is assumed. A liquid hydrogen target will not be used during the initial running since installation of the target would restrict cave access during development and monitoring of the target would distract from development tasks. Differential cross sections are taken from the VPI SM90 analysis. The solid angles are obtained from a NMS version of the PIANG program.

A total of 130 hours is needed for data runs. Assuming a $20 \%$ background from carbon in the $\mathrm{CH}_{2}$ target, a total of $130 / \sqrt{5}=60$ hours will be needed for background runs. It is assumed that, at least for the initial measurements in 1992 before the normal support apparatus for the NMS is built, it will be more efficient to make measurements at each of the five momenta for a given NMS geometry. Each beam change will require an estimated 4 hours for tuning and calibration of the monitors, an additional 60 hours. ''ositioning the spectrometer (and accurately measuring the position) is likely to be a tedious task during 1992, so an estimated 10 hours per angle change, or 30 hours, is estimated. Adding 20 hours for contingency brings the total beam request to 300 hours. This estimate assumes that the electronics and data acquisition software for the NMS will be completed as part of the NMS development program.

There are two uncertainties that affect the rate estimates significantly. The first is that all of the $120 \mathrm{Csl}$ crystals for the calorimeter may not be available next summer. If not, then the assumed solid angles decrease roughly by a factor of $L 2 H$, where $L(H)$ is the horizontal (vertical) dimension of the calorimeter arrays. The second is the uncertainty in the number of BGO converters that will be available. The final NMS configuration will have four converter planes in each arm, which will give $\varepsilon(\pi 0)$ of 0.53 for $\varepsilon_{c}=0.28$ in equation (9), a nominal value for $100 \mathrm{MeV} \gamma$ rays. Two converter planes in each arm reduces $\varepsilon(\pi \circ)$ to 0.23 This value is consistent with the assumption of $\varepsilon(\pi \circ) \varepsilon w f_{a b s} F_{\gamma^{\prime}} \tau_{L}=0.2$ in the rate calculations for $\varepsilon_{W} \sim 0.95, f_{a b s} \sim 0.9, F_{Y \gamma}=0.988$, 
and $\tau_{L}=0.95$. The po:ssibility of not even using converters is discussed in the next section.

\section{Compatibllity beitween NMS Development and the Proposed Measurements}

Several factors make measurements of cross sections for $\pi-p \rightarrow \pi \circ$ n a good "first physics" experiment to be performed in conjunction with NMS development. The rates at the forward and backward angles are very high (1\% statistics in a matter of hours, see Tables 1-3), the design energy resolution is not needed, and $\mathrm{CH}_{2}$ targets are simple to install and remove. For these reasons, the NMS committee decided at its meeting on 15 November to submit this proposal and another one to measure cross sections for $\pi^{-d} \rightarrow \pi$ onn (June Matthews, spokesperson) to the January, 1992, meeting of the PAC.

In principle, it is possible to detect $\pi^{\circ}$ 's without even using a converter system. The position determination of the individual gamma rays would then be reconstructed from the energy sharing in the Csl crystals of the calorimeter. This reconstruction would have a resolution of $\sim 3 \mathrm{~cm}$. The advantage is that $\varepsilon(\pi \circ) \varepsilon_{W}$ product becomes 1 in equation (9) and the uncertainty associated with this product is eliminated. The disadvantage is that the solid angle, $\Omega\left(\pi^{\circ}\right)$, is less well determined due to edge effects. The best of both worlds will be to take some, if not all, of the data without a converter requirement in the trigger in ordar to investigate the converter efficiencies as a function of $\gamma$ energy. This type of investigation is precisely what should be included in the NMS development program.

The concern in trying to perform a physics measurement concurrent with a development program is not to compromise either effort. The main difference envisioned if this proposal were submitted for an already-developed NMS would be to include some running time with a liquid hydrogen $\left(\mathrm{LH}_{2}\right)$ target. There are advantages to using solid targets, namely, a precise thickness determination (no bubbling corrections) and the ability to make the energy loss of the beam exactly the same for background runs by choosing an appropriate thickness for the carbon target. The carbon background is expected to be small at forward angles due to the difference in the $Q$ values in $\pi p$ and $\pi \mathrm{C}$ charge exchange. At backward angles the $\pi p$ signal will compete with quasielastic $\pi \mathrm{C}$ scattering. If this background is formidable then an addendum to this proposal will be submitted at a later date to allow for an independent measurement using $\mathrm{LH}_{2}$.

One concern that arose during the discussion of this proposal at the 15 November NMS meeting is that the accurate beam monitoring required might impede NMS development. Beam monitoring will be accomplished by having a combination of muon decay hodoscopes and ionization chambers that will be calibrated at low rate 
with beam counters. An independent determination using the $11 \mathrm{C}$ activation technique will also be used. These calibrations will require beam time but will not require anything out of the ordinary for the NMS. It is conceivable that software or electronics development for the NMS could proceed in parallel with the beam calibrations.

\section{References:}

[Ab89] V. V. Abaev, Proc. Third Int. Symp. on $\pi N$ and NN Physics, Leningrad, 147, 17-22 April, 1989.

[Ar85] R. A. Arndt, J. M. Ford and L. D. Roper, Phys. Rev. D32, 1085 (1985). The updated SM90 solution was obtained from the SAID program, available from the authors.

[Ba76] W. Bayer, et al., Nucl. Inst. and Meth. 134, 449 (1976).

[Be76] P. W. Bertin, et al., Nucl. Phys. B106, 341 (1976).

[Br86] J. T. Brack, et al., Phys. Rev. C34, 1771 (1986).

[Br88] J. T. Brack, et al., Phys. Rev. C38, 2427 (1988).

[Br 90] J. T. Brack, et al., Phys. Rev. C41, 2202 (1990).

[Be72] P. A. Berardo, et al., Phys. Rev. D6,756 (1972).

[Bu71] D. V. Bugg, et al., Nucl. Phys. B26, 588 (1971).

[Bu91] D. V. Bugg, $\pi$ N Newsletter No. 3, R. E. Cutkosky, G. Höhler, W. Kluge and

B. M. K. Nefkens, ed., 1, September 1991.

[Bu73] P. J. Bussey, et al., Nucl. Phys. B58, 363 (1973).

[Co89] J. R. Comfort and G. Burleson, spokespersons, LAMPF Prop. 1178 (1989).

[Co75] J. C. Comiso, et al., Phys. Rev. D12, 738 (1975).

[Fi86] D. H. Fitzgerald, et al., Phys. Rev. C34, 619 (1986).

[Ga84] F. C. Gaille, et al., Phys. Rev. D30, 2408 (1984).

[Ho79] G. Höhler, F. Kaiser, R. Koch and E. Pietarinen, Handbook of Pion-Nucleon Scattering, Physics Data No.12-1 (1979).

[Hö82] G. Höhler, Pion-Nucleon Scattering, in Landolt-Börnstein 9b, Springer-Verlag (1982).

[Hö90] G. Höhler and J. Stahov, $\pi N$ Newsletter No. 2, G. Höhler, W. Kluge and B. M. K. Nefkens, ed., 42, May 1990.

[Je77] R. F. Jenefsky, et al., Nucl. Phys. A290, 407 (1977).

[K080] R. Koch and E. Pletarinen, Nucl. Phys. A336, 331 (1980).

[McF83] W. K. McFarlane, et al., Phys. Rev. Lett. 51, 249 (1983).

[Par91] S. Parry, H. Park, M. Rawool-Sullivan and C. Morris, private communication.

[Pav91] M. Pavfan, et at., presented at the Fourth Int. Symp. on $\pi N$ Physics, Bad Honnef, Germany, 9-13 September, 1991.

[Pe78] E. Pedroni, et al., Nucl. Phys. A300, 321 (1978).

[Sa87] M. E. Sadler, et al., Phys. Rev. D35, 2718 (1987). 


\begin{tabular}{|c|c|c|c|c|c|c|c|c|c|c|c|}
\hline $\begin{array}{l}P_{\pi} \\
229\end{array}$ & $\begin{array}{c}T_{\pi} \\
128.6\end{array}$ & $\begin{array}{r}N(\pi) / \mathrm{s} \\
5.0 \mathrm{E}+0\end{array}$ & & $\begin{array}{l}\mathrm{N}_{H}(\# / \mathrm{cr} \\
2.0 \mathrm{E}+2\end{array}$ & & $\varepsilon\left(\pi^{\circ}\right)$ & $f_{a b s} F$ & & & & \\
\hline$\theta_{\pi^{\circ}}^{L}$ & $\theta_{\pi^{\circ}}$ & $\cos \theta_{\pi^{\circ}}$ & $E_{\pi}{ }^{\circ}$ & $P_{\pi} 0$ & $T_{\pi^{0}}$ & $1 / \mathrm{J}$ & $\eta$ & $d \sigma / d \Omega$ & $\Omega\left(\pi^{0}\right)$ & Rate(/ & ) Time(hr) \\
\hline 0 & 0.0 & 1.00 & 266.9 & 230.2 & 131.9 & 1.58 & 60.8 & 2.84 & & & \\
\hline 20 & 25.0 & 0.91 & 263.6 & 226.4 & 128.6 & 1.53 & 61.6 & 2.49 & 6.53 & 0.72 & 3.8 \\
\hline 40 & 49.3 & 0.65 & 254.6 & 215.8 & 119.6 & 1.40 & 64.0 & 1.77 & & & \\
\hline 60 & 72.3 & 0.30 & 242.3 & 201.2 & 107.3 & 1.22 & 67.7 & 1.36 & & & \\
\hline 80 & 93.6 & -0.06 & 229.3 & 185.4 & 94.3 & 1.04 & 72.1 & 1.69 & 5.60 & 0.14 & 19.5 \\
\hline 100 & 113.3 & -0.39 & 217.6 & 170.6 & 82.6 & 0.88 & 76.7 & 2.75 & & & \\
\hline 140 & 148.3 & -0.85 & 201.4 & 149.5 & 66.5 & 0.67 & 84.1 & 5.51 & & & \\
\hline 160 & 164.4 & -0.96 & 197.5 & 144.2 & 62.5 & 0.62 & 86.2 & 6.41 & 6.29 & 1.70 & 1.6 \\
\hline 180 & 180.0 & -1.00 & 196.2 & 142.4 & 61.2 & 0.61 & 87.0 & 6.77 & & & \\
\hline & & & & & & & Tota & ata hou & & & 25.0 \\
\hline
\end{tabular}

Table 1. Kinematics and estimated rates at $229 \mathrm{MeV} / \mathrm{c}$ for $\pi \cdot \mathrm{p} \rightarrow \pi$ on at $\theta_{\pi^{\circ}}^{\mathrm{L}}=20^{\circ}, 80^{\circ}$ and $160^{\circ}$. See equation (8-10) in the text for a description of the quantities. The beam rate is limited to $5 \cdot 106 / \mathrm{s}$ for normalization purposes and to obtain small $\Delta \mathrm{p} / \mathrm{p}$. The hydrogen density is that for $2.5 \mathrm{~cm}$ of $\mathrm{CH}_{2}$. At a distance of one meter, the NMS will subtend an angular interval approximately $\pm 20^{\circ}$ from the central lab angle. The kinematics are also shown for these angles to indicate the range of $\cos \theta_{\pi^{\circ}}$ that will be accepted. The differential cross sections were obtained from the VPI SM90 analysis [Ar85]. The solid angle, $\Omega(\pi \circ)$, is the full acceptance at the given angle, but the rate is computed per interval of 0.05 for $\cos \theta_{\pi}{ }^{\circ}$. The time is that required to obtain 10,000 counts in the central interval. 


\begin{tabular}{|c|c|c|c|c|c|c|c|c|c|c|c|}
\hline $\begin{array}{l}P_{\pi^{-}} \\
260\end{array}$ & $\begin{array}{l}\mathrm{T}_{\pi} \\
155.5\end{array}$ & \multicolumn{2}{|c|}{$\begin{array}{l}N(\pi) / \mathrm{s} \\
5.0 \mathrm{E}+06\end{array}$} & \multicolumn{2}{|c|}{$\begin{array}{l}\mathrm{N}_{H}\left(\# / \mathrm{cm}^{2}\right) \\
2.0 \mathrm{E}+23\end{array}$} & \multicolumn{3}{|c|}{$\underset{0.2}{\varepsilon(\pi \circ) \varepsilon w f_{a b s} F_{\gamma} \tau_{L}}$} & \multirow[b]{2}{*}{$\Omega\left(\pi^{\circ}\right)$} & \multirow[b]{2}{*}{ Rate(/s) } & \multirow[b]{2}{*}{ ) Time(hr) } \\
\hline$\theta_{\pi^{\circ}}^{L}$ & $\theta_{\pi^{0}}$ & $\cos \theta_{\pi^{\circ}}$ & $E_{\pi^{\circ}}$ & $P_{\pi}$ & $T_{\pi}$ & $1 / \mathrm{J}$ & $\eta$ & $d \sigma / d \Omega$ & & & \\
\hline 0 & 0.0 & 1.00 & 293.8 & 261.0 & 158.8 & 1.64 & 54.7 & 5.60 & & & \\
\hline 20 & 25.5 & 0.90 & 289.5 & 256.1 & 154.6 & 1.58 & 55.6 & 4.86 & 6.69 & 1.45 & 1.9 \\
\hline 40 & 50.1 & 0.64 & 278.0 & 243.1 & 143.1 & 1.43 & 58.1 & 3.34 & & & \\
\hline 60 & 73.3 & 0.29 & 262.5 & 225.2 & 127.6 & 1.23 & 61.9 & 2.22 & & & \\
\hline 80 & 94.7 & -0.08 & 246.3 & 206.1 & 111.4 & 1.03 & 66.4 & 2.27 & 6.04 & 0.21 & 13.5 \\
\hline 100 & 114.2 & -0.41 & 231.9 & 188.6 & 36.9 & 0.87 & 71.2 & 3.43 & & & \\
\hline 140 & 148.8 & -0.86 & 212.3 & 163.9 & 77.4 & 0.65 & 78.9 & 6.82 & & & \\
\hline 160 & $164.6^{\prime}$ & -0.96 & 207.6 & 157.7 & 72.6 & 0.60 & 81.1 & 7.96 & 5.61 & 1.87 & 1.5 \\
\hline 180 & 180.0 & -1.00 & 206.0 & 155.6 & 71.0 & 0.58 & 81.9 & 8.41 & & & \\
\hline & & & & & & & Tota & a hours & & & 16.9 \\
\hline
\end{tabular}

$P_{\pi^{-}} \quad T_{\pi^{-}} \quad N(\pi) / s \quad N_{H}\left(\# / c^{2}\right) . \quad \varepsilon(\pi \circ) \varepsilon W^{f} f_{a b s} F_{\gamma} \tau_{L}$

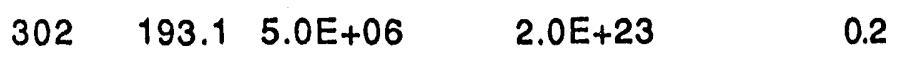

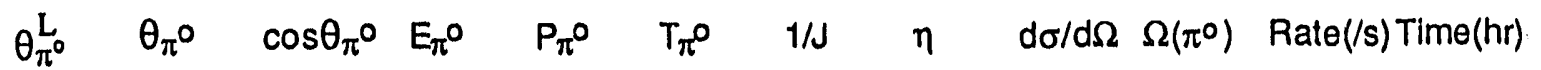

$\begin{array}{lllllllll}0 & 0.0 & 1.00 & 331.4 & 302.7 & 196.4 & 1.72 & 48.1 & 7.46\end{array}$

$\begin{array}{llllllllllll}20 & 26.1 & 0.90 & 325.7 & 296.4 & 190.7 & 1.65 & 49.0 & 6.37 & 7.04 & 2.01 & 1.4\end{array}$

$\begin{array}{lllllllll}40 & 51.2 & 0.63 & 310.4 & 279.5 & 175.4 & 1.48 & 51.6 & 4.13\end{array}$

$\begin{array}{lllllllll}60 & 74.6 & 0.27 & 290.0 & 256.6 & 155.0 & 1.25 & 55.5 & 2.35\end{array}$

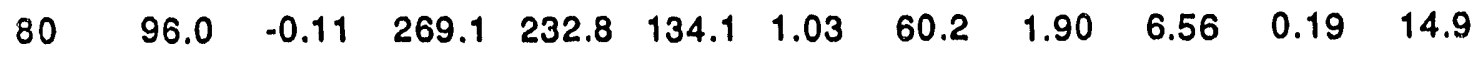

$\begin{array}{lllllllll}100 & 115.5 & -0.43 & 250.8 & 211.3 & 115.8 & 0.85 & 65.1 & 2.63\end{array}$

$\begin{array}{lllllllll}140 & 149.5 & -0.86 & 226.4 & 181.8 & 91.4 & 0.62 & 73.2 & 5.24\end{array}$

$\begin{array}{llllllllllll}160 & 165.0 & -0.97 & 220.5 & 174.4 & 85.6 & 0.57 & 75.5 & 6.12 & 5.53 & 1.42 & 2.0\end{array}$

$\begin{array}{lllllllll}180 & 180.0 & -1.00 & 218.6 & 172.0 & 83.6 & 0.56 & 76.2 & 6.48\end{array}$

Total data hours $=\quad 18.3$

Table 2. Kinematics and estimated rates at 260 and $302 \mathrm{MeV} / \mathrm{c}$. See caption of Table 1 for details. 
$\begin{array}{llllc}P_{\pi^{*}} & T_{\pi *} & N(\pi) / s & N_{H}\left(\# / \mathrm{cm}^{2}\right) & \varepsilon\left(\pi^{0}\right) \varepsilon W_{\text {abs }} F_{\gamma} \tau_{L} \\ 337 & 225.2 & 5.0 E+06 & 2.0 E+23 & 0.2\end{array}$

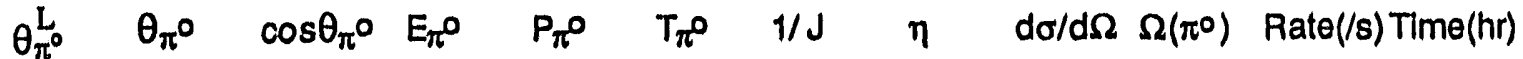

$\begin{array}{lllllllll}0 & 0.0 & 1.00 & 363.5 & 337.5 & 228.5 & 1.79 & 43.6 & 6.80\end{array}$

$\begin{array}{llllllllllll}20 & 26.6 & 0.89 & 356.3 & 329.8 & 221.4 & 1.71 & 44.5 & 5.68 & 7.37 & 1.88 & 1.5\end{array}$

$\begin{array}{lllllllll}40 & 52.1 & 0.61 & 337.5 & 309.3 & 202.5 & 1.51 & 47.2 & 3.46\end{array}$

$\begin{array}{lllllllll}60 & 75.7 & 0.25 & 312.7 & 282.0 & 177.7 & 1.26 & 51.1 & 1.74\end{array}$

$\begin{array}{lllllllllllll}80 & 97.1 & -0.12 & 287.6 & 254.0 & 152.6 & 1.03 & 56.0 & 1.16 & 6.68 & 0.12 & 23.9\end{array}$

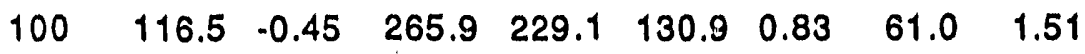

$\begin{array}{lllllllll}140 & 150.1 & -0.87 & 237.5 & 195.4 & 102.5 & 0.60 & 69.3 & 3.04\end{array}$

$\begin{array}{llllllllllll}160 & 165.3 & -0.97 & 230.7 & 187.1 & 95.7 & 0.55 & 71.6 & 3.56 & 5.61 & 0.84 & 3.3\end{array}$

$\begin{array}{lllllllll}1.80 & 180.0 & -1.00 & 228.5 & 184.4 & 93.5 & 0.54 & 72.4 & 3.76\end{array}$

Total data hours $=$

28.7

$P_{\pi^{-}} \quad T_{\pi^{*}} \quad N(\pi) / s \quad N_{H}\left(\# / \mathrm{cm}^{2}\right) \quad \varepsilon(\pi \circ) \varepsilon W^{\prime} f_{a b s} F_{\gamma /} \tau_{L}$

$\begin{array}{lllll}359 & 245.6 & 5.0 \mathrm{E}+06 & 2.0 \mathrm{E}+23 & 0.2\end{array}$

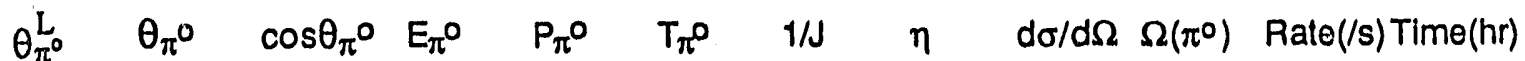

$\begin{array}{lllllllll}0 & 0.0 & 1.00 & 383.9 & 359.4 & 248.9 & 1.84 & 41.2 & 6.22\end{array}$

$\begin{array}{llllllllllll}20 & 26.9 & 0.89 & 375.8 & 350.7 & 240.8 & 1.75 & 42.1 & 5.10 & 7.60 & 1.74 & 1.6\end{array}$

$\begin{array}{lllllllll}40 & 52.6 & 0.61 & 354.5 & 327.8 & 219.6 & 1.53 & 44.8 & 2.98\end{array}$

$\begin{array}{lllllllll}60 & 76.4 & 0.24 & 326.8 & 297.6 & 191.8 & 1.27 & 48.8 & 1.39\end{array}$

$\begin{array}{lllllllllllll}80 & 97.8 & -0.14 & 299.0 & 266.8 & 164.0 & 1.02 & 53.7 & 0.82 & 6.78 & 0.08 & 33.4\end{array}$

$\begin{array}{lllllllll}100 & 117.1 & -0.46 & 275.1 & 239.7 & 140.1 & 0.83 & 58.8 & 1.02\end{array}$

$\begin{array}{lllllllll}140 & 150.5 & -0.87 & 244.1 & 203.4 & 109.2 & 0.59 & 67.1 & 2.07\end{array}$

$\begin{array}{llllllllllll}160 & 165.5 & -0.97 & 236.8 & 194.6 & 101.8 & 0.54 & 69.5 & 2.42 & 5.71 & 0.58 & 4.8\end{array}$

$\begin{array}{lllllllll}180 & 180.0 & -1.00 & 234.4 & 191.7 & 99.4 & 0.52 & 70.3 & 2.56\end{array}$

Total data hours $=\quad 39.8$

Table 3. Kinematics and estimated rates at 337 and $359 \mathrm{MeV} / \mathrm{c}$. See caption of Table 1 for details. 

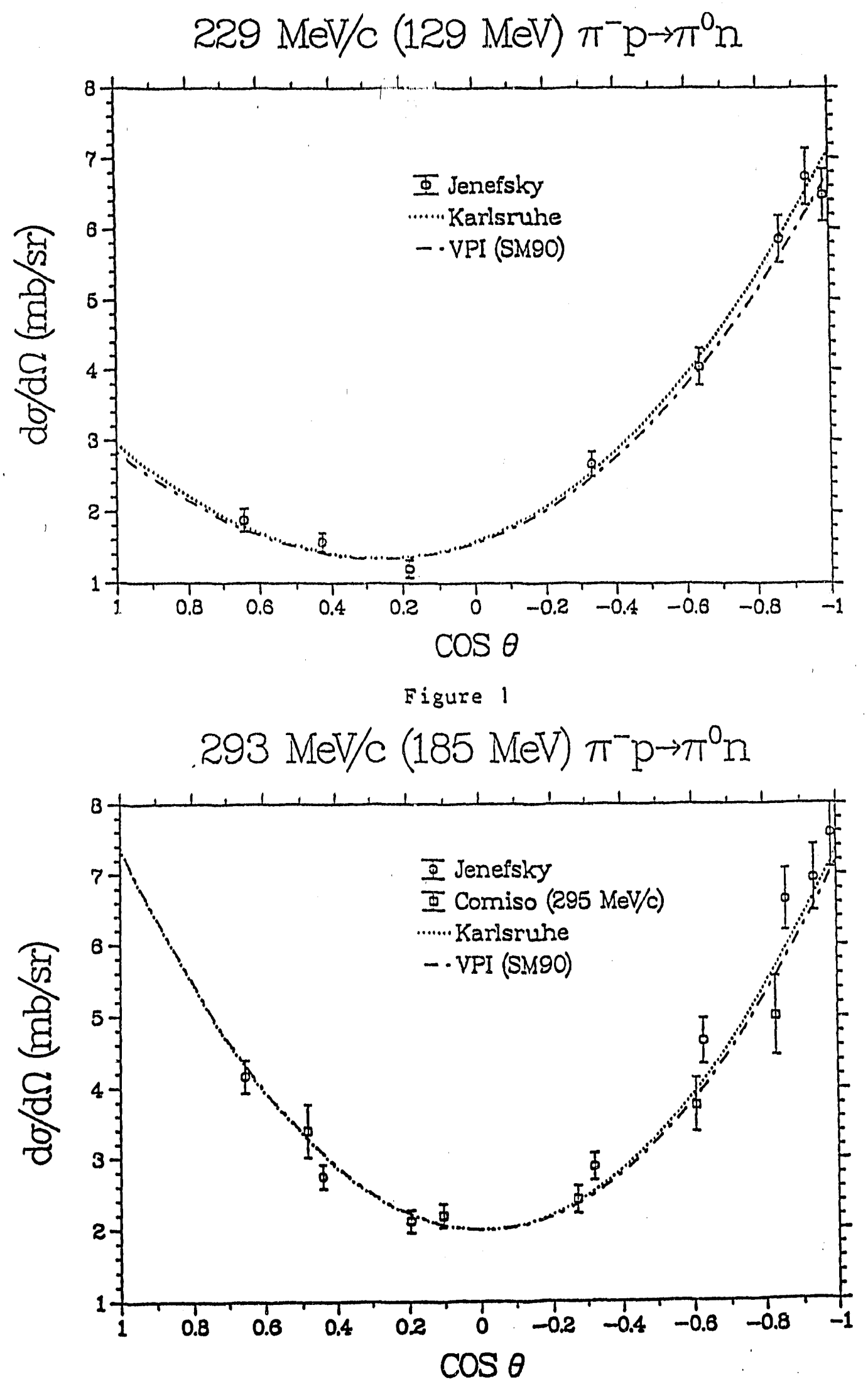

Figure 2 


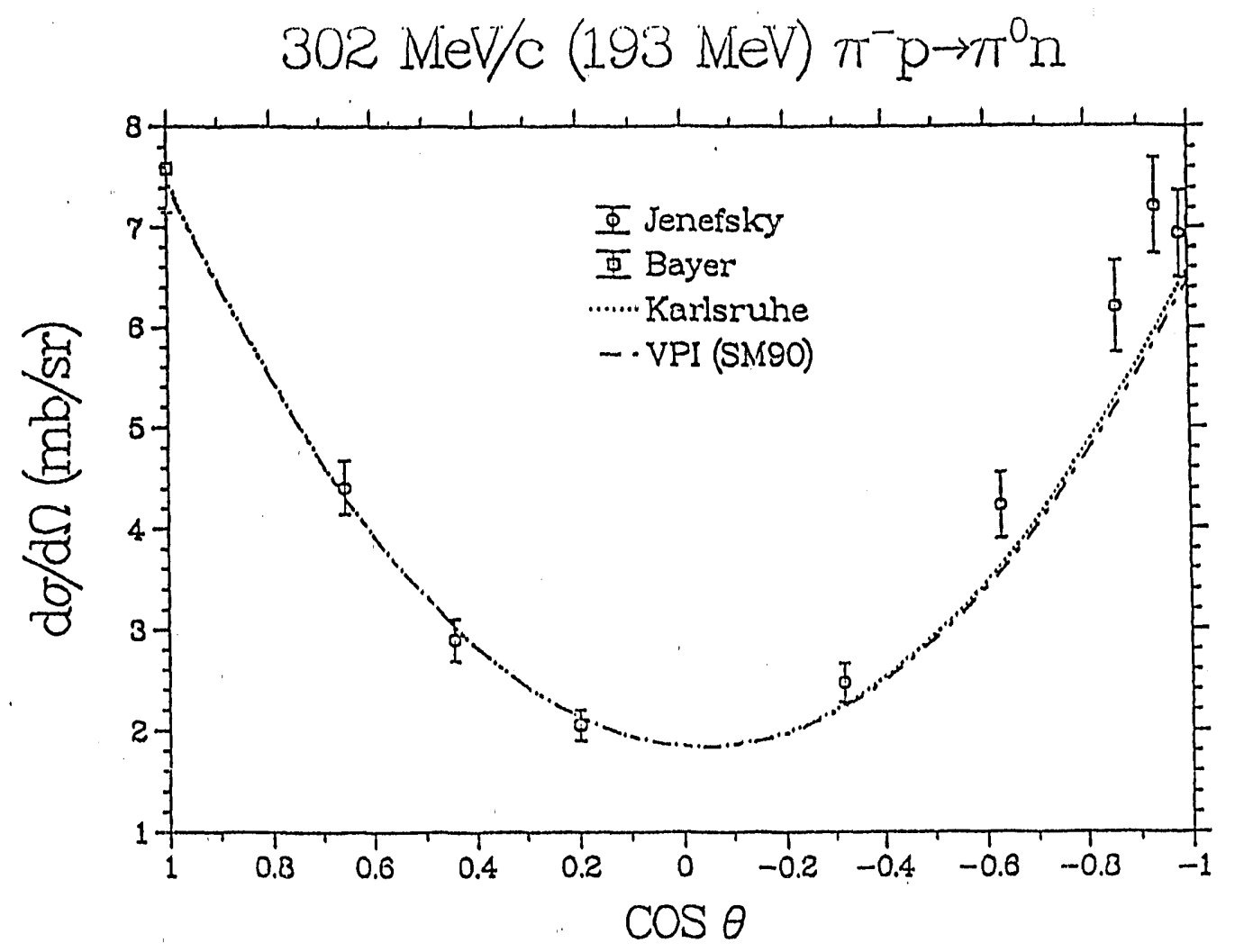

Figure 3

$359 \mathrm{MeV} / \mathrm{c}(246 \mathrm{MeV}) \pi^{-} \mathrm{p} \rightarrow \pi^{0} \mathrm{n}$

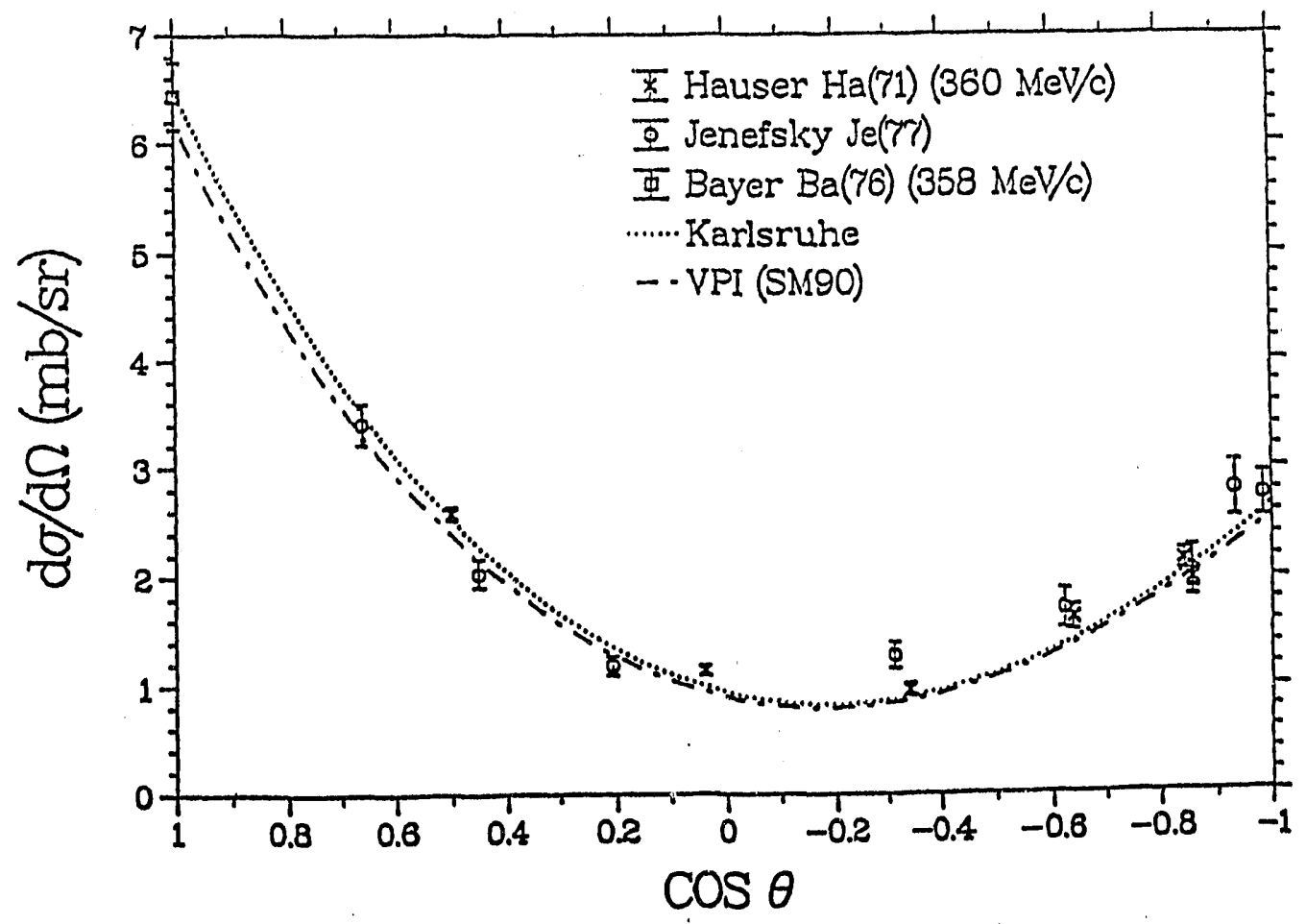

Figure 4 


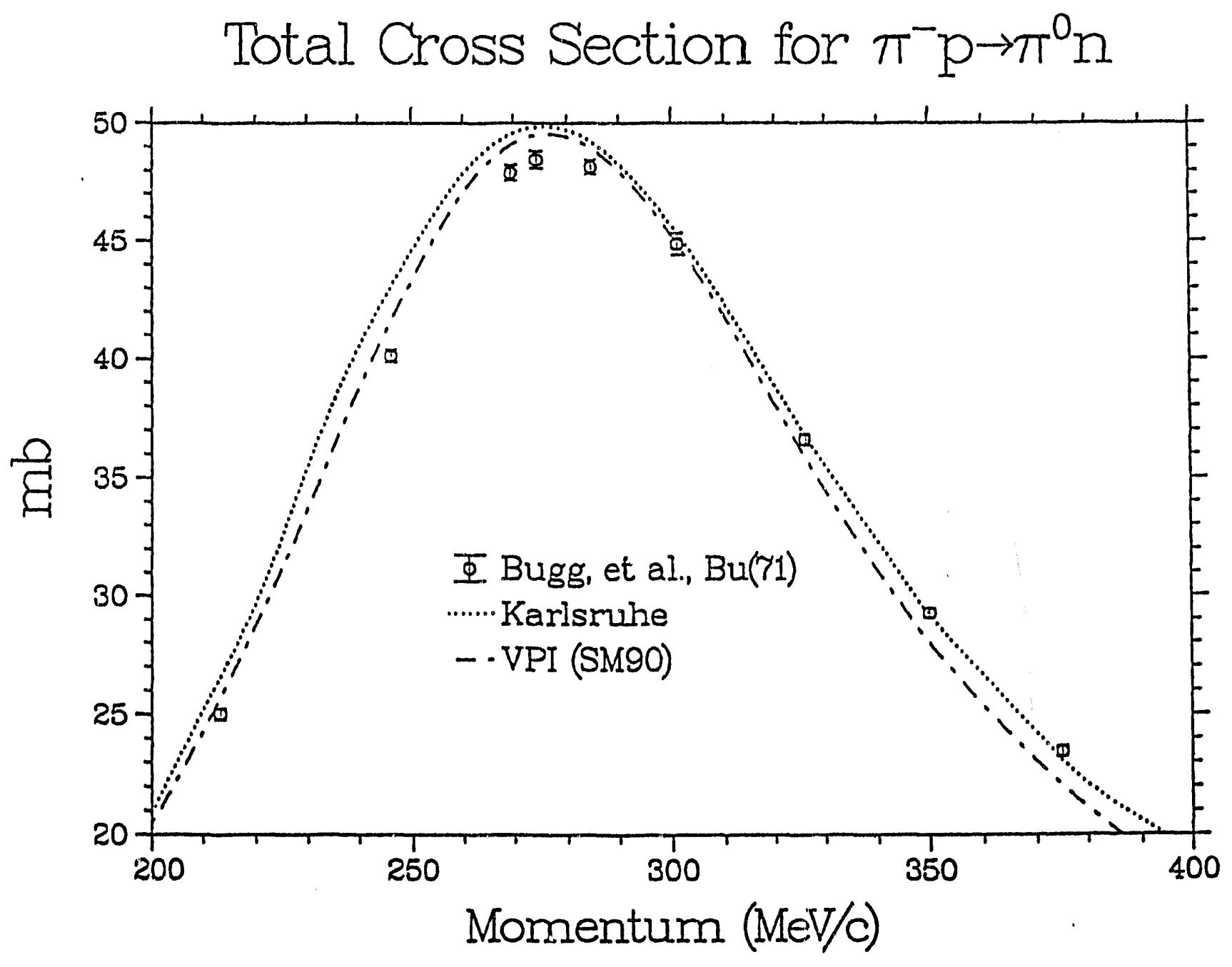

Figure 5 


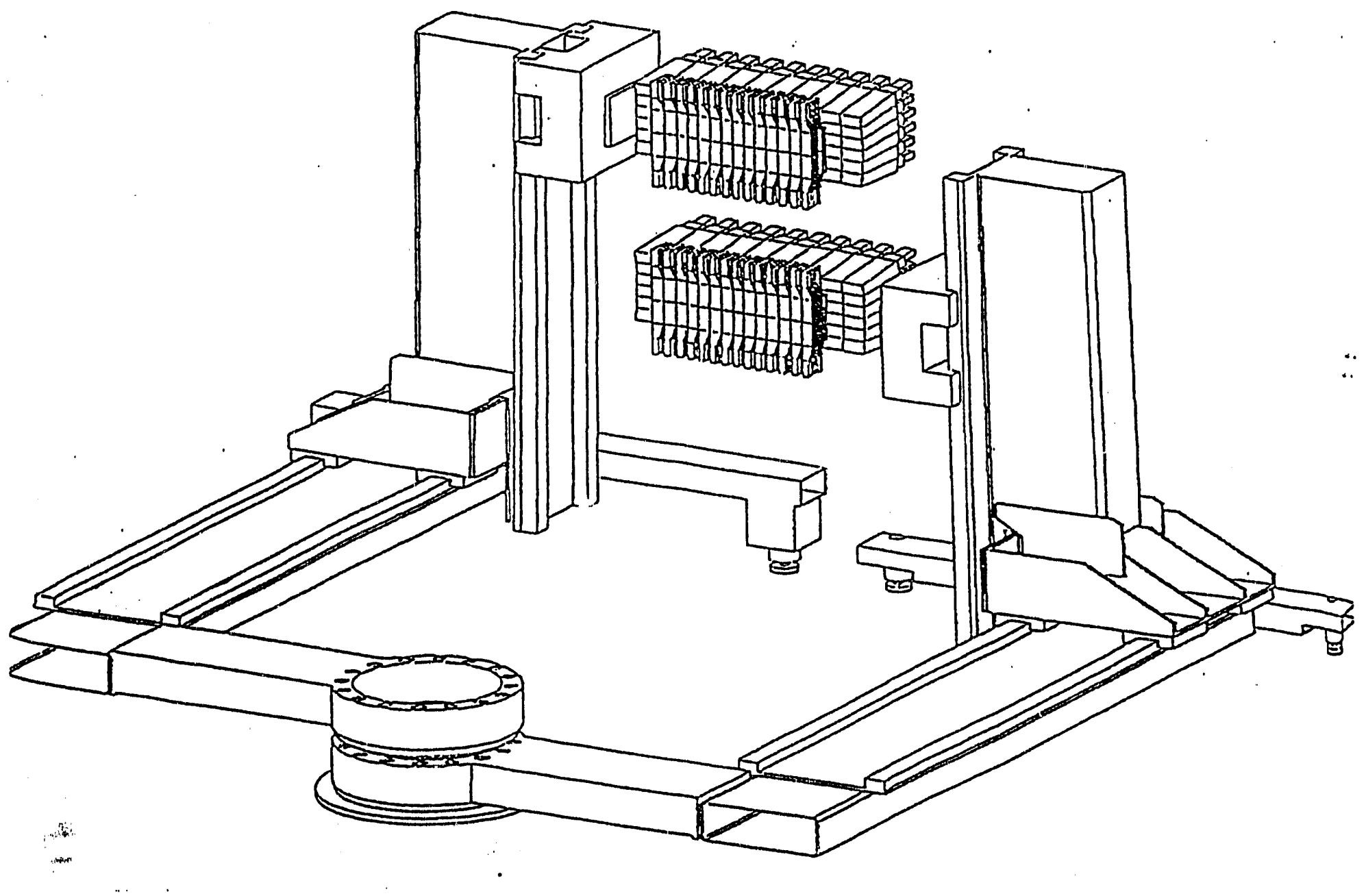

Figure 6 


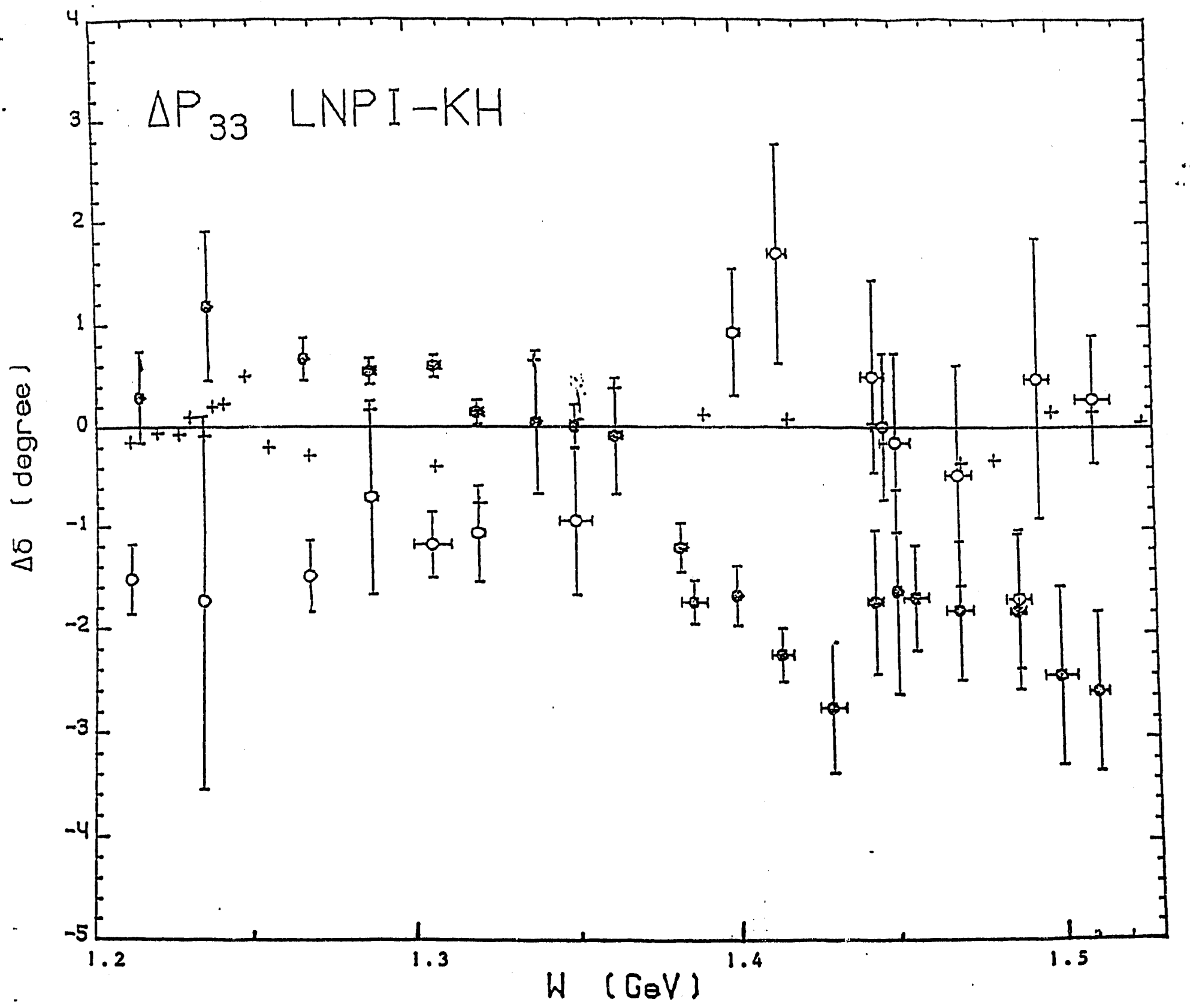

Figure 7 


\section{D. $\pi N$ Partial-Wave Analysis and the $\pi N N$ Coupling Constant}

The rate of inclusion of our complete data set into new partial wave analyses (PWA's) has been disappointing. The most recent analyses that cover an extended interval in the resonance region for both $\pi+p$ and $\pi$-p scattering are the VPI1, KarlsruheHelsinki2, and CMU-LBL3 analyses. Constraints common to all these analyses are unitarity, isospin invariance and forward dispersion relations. A unique solution was accomplished by the latter two efforts by additional dispersion relations which follow from the Mandelstam hypothesis that the amplitudes are analytic functions of $s$ and $t$. The analyses differ in their treatments of electromagnetic effects, the procedures in which the constraints are applied, and in their experimental databases. The compilation of non-strange, isospin-1/2 and $-3 / 2$ baryons of the Particle Data Group4 is derived from the Karlsruhe and CMU-LBL analyses, each of which was last published in 1980. The Karlsruhe analysis extends to $10 \mathrm{GeV} / \mathrm{c}$ and the CMU-LBL to $2.5 \mathrm{GeV} / \mathrm{c}$. The parameterization used by the VPI group gives the flexibility of updating the analysis periodically via the SAID facility. This analysis has recently been extended to 2.2 $\mathrm{GeV} / \mathrm{c}$.

Collaboration between experimentalists and theorists is needed in order to make the best evaluations of conflicting data sets and to propose new experiments based on the results. Since the summer of 1989, Sadler has actively worked with various PWA efforts (G. Höhler in Karlsruhe, Y. Stahov in Tuzla, Yugoslavia, and V. Abaev in St. Petersburg) with the primary objective of incorporating the recent data into an updated analysis of the Karlsruhe type. Evaluation of databases and the use of existing analyses as "data" to evaluate fundamental quantities, such as the $\pi \mathrm{N}$ sigma term or the $\pi \mathrm{NN}$ coupling constant, are also important. It was just such an investigation, performed by Stahov, Abaev and Sadler during a one-month long visit in 1990 , that led to following comment published last month which is included below.

The travel expenses for this work are partially funded by U.S.D.O.E. under a joint U.S.-Yugoslavia program for cooperative research. Due to the unstable situation in Yugoslavia the U.S. portion of this grant has not been used at this point; hopefully, this situation will get better and travel to that country can be resumed for work on this project. Work is also being done on finding ways of using these funds to get the researchers together in Abilene again during 1992-93.

\section{References}

1. R.A. Arndt, et al., Phys. Rev. D32, 1085 (1985).

2. G.H. Höhler, et al., Phys. Data 12-1, Karlsruhe (1979).

R. Koch, et al., Nucl. Phys. A336, 331 (1980).

3. R.E Cutkosky, et al., Phys. Rev. D20, 2804 (1979); D20, 2839 (1979).

4. Review of Particle Properties, Phys. Lett. B239 (1990). 


\section{E. Measurement of the Differential Cross Sections for $\pi \cdot p \rightarrow \pi$ on and $\pi-p \rightarrow \eta n$ at the St. Petersburg Nuclear Physics Institute}

\section{Summary of Experimental Program}

Physicists from the St. Petersburg (formerly Leningrad) Nuclear Physics Institute (SPNPI), the University of California at Los Angeles (UCLA), and Abilene Christian University (ACU) have formed the Collaboration of United States and Soviet Union Ehysicists (CUSP). (The collaboration will have to be renamed due to the recent political changes in Russia.) The collaboration plans to utilize the existing zerodegree pion channel at the $1 \mathrm{Gev}$ proton synchrocyclotron at SPNPI to measure neutral-particle final states from $\pi$-p interactions. The primary detectors to be used are existing neutron counters from UCLA and lead-glass Cherenkov detectors for $\gamma$ rays which were obtained from Saclay. Different aspects of the planned program are summarized in the following four paragraphs.

The collaboration derived its name from the planned measurement of the cusp in the differential cross sections for the reaction $\pi \cdot p \rightarrow \pi$ on at $P_{\pi}=657-717 \mathrm{MeV} / \mathrm{c}$. Measurements of sufficient momentum resolution and accuracy are needed to deduce the effect of the opening of the $\eta n$ channel on the charge exchange cross section. A cusp, or discontinuity in the slope of the cross section as a function of momentum (at fixed angle), is expected at the threshold $(687 \mathrm{MeV} / \mathrm{c})$ for $\eta$ production. The experiment involves binning the pion beam at SPNPI, which has a full acceptance of $\Delta p / p=6.0 \%$, into eight intervals of $0.7 \%$ using a scintillator hodoscope placed in the channel where the beam is momentum dispersed. This hodoscope is presently being rebuilt and is scheduled to be installed in the SPNPI beam line in March. The experimental layout along with Monte-Carlo calculations of the acceptance for $\pi \cdot p \rightarrow$ $\pi \circ n$ (performed at ACU) were detailed in the 1991 DOE Renewal and are not reproduced here.

Measurement of the reaction $\pi-p \rightarrow \eta n$ is of interest as a calibration mechanism for the cusp measurement. Observation of this final state at threshold allows a direct calibration of the absolute momentum and $\Delta p / p$ of the beam (assuming a given mass of the $\eta$ meson) or, alternatively, an independent measurement of the $\eta$ mass. (depending on the accuracy of the calibration of the beam momentum). Preliminary measurements, i.e., a momentum scan of $\eta$ production, were completed by the collaboration during July, 1991 and are reported in the next section. These measurements marked the first time that $\eta$ production has been identified at SPNPI.

Measurements of $d \sigma / d \Omega$ for $\pi-p \rightarrow \eta n$ are also of intrinsic interest. This cross section is large, so by time reversal, the $\eta$ interaction with the nucleon is also large. This final-state interaction will interfere with the dominantly s-wave production of the $\eta$ near threshold, giving a t dependence. Binnie, et al.1, measured the t dependence by 
varying the incident beam momentum. Multiple neutron counters will allow this measurement to be done by varying the angular distribution of recoil neutrons at momenta above threshold. Information on the $\eta N$ interaction could then be extracted.

Depending on the success of these programs and the ability to obtain an accurate absolute efficiency calibration of the neutron counters, a third experimental program is the measurement of differential cross sections for $\pi-p \rightarrow \pi$ on at $P_{\pi}=400$ $700 \mathrm{MeV} / \mathrm{c}$. Improvements over previous measurements should be possible due to improved neutron and gamma detectors. Precise differential-cross-section data are needed to investigate charge splitting in the pion-nucleon $(\pi \mathrm{N})$ system and to deduce the parameters (mass, width and inelasticity) of the $P_{11}$ and $S_{11}$ resonances.

Finally, a fourth aspect of the collaboration is the partial wave analysis (PWA) of $\pi \mathrm{N}$ scattering data. The PWA uses results from each of the three experimental programs described. An ongoing PWA effort is needed to complement these programs, to incorporate the recent data from LAMPF (UCLA-ACU-GWU collaboration) and SPNPI in this energy region, and to prepare for future $\pi \mathrm{N}$ experiments in the overlapping resonance region that will be forthcoming from a future meson facility (e.g., PILAC or KAON). A state-of-the-art analysis will involve a pruned database including the most reliable data, the incorporation of complete sets of data at a single momentum (including measurements of the $A$ and $R$ spin rotation parameters obtained at both LAMPF and SPNPI), and inclusion of constraints from dispersion theory to obtain a unique solution.

Discussion of this program started when B. M. K. Nefkens (UCLA) and M. E. Sadler (ACU) participated in the Third International Symposium on Pion-Nucleon Physics held at SPNPI in April, 1989. An invitation was offered and accepted to visit Gatchina, near St. Petersburg, in July, 1990, to form the collaboration and to plan the experimental program. The primary experimentalists from SPNPI are S. P. Kruglov, I. V. Lopatin and V. S. Bekrenev. The partial wave analysis is largely done by V. V. Abaev.

Perhaps the first question that arises in discussing this program is why could the measurements not be performed at the Clinton $P$. Anderson meson physics facility at Los Alamos (LAMPF), which has typical pion beam intensities two orders of magnitude higher than what is available at SPNPI. The answer is that the energy of the primary proton beam at LAMPF is $0.8 \mathrm{GeV}$ and at SPNPI it is $1.0 \mathrm{GeV}$. Also, pions are obtained at $20^{\circ}$ with respect to the proton beam at LAMPF compared to $0^{\circ}$ at SPNPI. The threshold for $\pi-p \rightarrow \eta n$ occurs at $P_{\pi}=687 \mathrm{MeV} / c$ and measurement of the cusp requires measuring the differential cross section over an interval of $\sim 30 \mathrm{MeV} / \mathrm{c}$ on either side of this value. This momentum is near the upper limit of what is available at the highest momentum channel $\left(P^{3}\right)$ at LAMPF. Extending the momentum range above the cusp would be an exercise in futility at $P 3$. Using the full acceptance $(\Delta p / p=$ $6 \%$ ) of the pion channel at SPNPI, the available $\pi$-intensities at SPNPI are $3.0 \cdot 105 / \mathrm{s}$ 
at $687 \mathrm{MeV} / \mathrm{c}$ and $1.7 \cdot 105 / \mathrm{s}$ at $725 \mathrm{MeV} / \mathrm{c}$. Although still rather low, these intensities are sufficient to perform the measurements. Development work with different pion production targets and possible new quadrupoles is in progress at SPNPI to increase the pion beam intensity.

\section{Reference:}

1. D. M. Binnie, et al., Phys. Rev. D8, 2789 (1973).

\section{Feasibility Study of $\eta$ Production}

Scott Garner contributed to this section.

A feasibility study for $\eta$ production in the SPNPI beam was completed during the period of 5-19 July, 1991. Participants from SPNPI were S. P. Kruglov, I. V. Lopatin, V.S. Bekrenev, V. V. Abaev, E. A. Filimonov, V. V. Sumachev, A. B. Gridnev, L. V. Lapochkina, A. Yu. Mayorov and N. G. Kozlenko; from UCLA were B. M. K. Nefkens, D. White and J. Price; from ACU were M. E. Sadler, J. Phillips and S. Garner. Initial plans were to make a preliminary measurement of the cusp for the charge exchange reaction. However, the beam hodoscope needed to perform the $\Delta p / p$ binning would have required several days to install, necessitating the removal and reinstallation of shielding along the beam line. In addition, there were not enough CAMAC TDC's available for all the needed neutron-gamma coincidences. Therefore, the decision was made to identify $\eta$ production using only the neutron counters, as described below. A great deal was learned about what will be needed to complete. successfully the experimental program outlined in the preceding section.

The neutron counters used to gather these data were placed at $1.034^{\circ}, 2.116^{\circ}$, $3.196^{\circ}, 5.289^{\circ}, 6.360^{\circ}$ and $7.427^{\circ}$ from the beam axis at a distance of $4.71 \mathrm{~m}$. The $\pi$ : beam was elliptical, $1.5 \mathrm{~cm}$ high by $3.5 \mathrm{~cm}$ wide (FWHM) with $\Delta \mathrm{p} / \mathrm{p}=0.75 \%$. The beam momenta ran from $670 \mathrm{MeV} / \mathrm{c}$ to $700 \mathrm{MeV} / \mathrm{c}$ in $5 \mathrm{MeV} / \mathrm{c}$ steps and then at $710 \mathrm{MeV} / \mathrm{c}$ and $720 \mathrm{MeV} / \mathrm{c}$. The neutron counters were $8.9 \mathrm{~cm}$ wide by $25.4 \mathrm{~cm}$ high. The liquid hydrogen target was a cylinder $10 \mathrm{~cm}$ in diameter.

Time-of-flight (TOF) spectra between the incident $\pi$-beam and the neutron counter signals were accumulated for all six counters simultaneously. The timing resolution of the discriminators used for the neutron signals was inadequate to show sharp timing peaks, but the prompt peak corresponding to $\gamma$ production was identified and timing positions for neutron signals from $\pi-p \rightarrow \eta n$ were determined. The yields shown in Table 1 were determined by summing the counts in a 5-ns interval around these positions. Since the first three bins in any column in Table 1 correspond to momenta below the $\eta$ threshold, their average was taken and subtracted from every bin in that column to compensate for the background. Table 2 shows these 
background-corrected data which are plotted (with error bars) in Fig. 1. The plots labelled A1-A7 correspond to the neutron counter angles near $1^{\circ}$ to near $7^{\circ}$.

The calculations used in making the points connected by lines without the error bars were generated by a Monte-Carlo program written by Jason Phillips. This program calculates the acceptance of the counters for neutrons from $\pi-p \rightarrow \eta n$ for the geometry used. The calculations were done in $1 \mathrm{MeV} / \mathrm{c}$ steps from $686 \mathrm{MeV} / \mathrm{c}$ to 705 $\mathrm{MeV} / \mathrm{c}$ and in $5 \mathrm{MeV} / \mathrm{c}$ steps to $720 \mathrm{MeV} / \mathrm{c}$. The total yield depends on (acceptance)•(production cross section). The latter, in turn, should increase linearly with the $\eta \mathrm{cm}$ momentum. Finally, the results of the (acceptance) $\cdot(\eta \mathrm{cm}$ momentum) product were averaged over the momentum biie of $0.75 \%$ and normalized to the experimental results using the numbers in the highest three bins. These numbers are compared to the experimental results in Fig. 1 for all six angles (A1-A7).

The statistical errors shown in Fig. 1 are not impressive. The spectra required nominally one hour of beam per momentum. Better timing of the neutron counter coincidences, through the use of constant-fraction discriminators, would greatly enhance the signal-to-background ratio in the TOF spectra. Nevertheless, the results show that $\eta$ production has been observed at SPNPI. Moreover, the comparisons with the Monte-Carlo calculations indicate that the nominal momentum of the SPNPI beam is 3-7 MeV/c $(0.5-1 \%)$ higher than the actual value.

The discriminators and CAMAC TDC's needed to measure the neutron timing are presently being considered as part of a $\$ 24,000$ additional request to last year's budget. These modules would then be used for the tomography setup discussed in Sec. III below when it is transported to ACU. 


$\begin{array}{cllllll}P(M \Theta V / c) & A 1 & A 2 & A 3 & A 5 & A 6 & A 7 \\ 670 & 172 & 93 & 131 & 122 & 112 & 101 \\ 675 & 137 & 101 & 103 & 115 & 105 & 120 \\ 680 & 170 & 101 & 132 & 145 & 110 & 100 \\ 685 & 188 & 142 & 173 & 160 & 129 & 125 \\ 690 & 219 & 170 & 203 & 175 & 170 & 147 \\ 695 & 220 & 207 & 219 & 238 & 195 & 178 \\ 700 & 180 & 152 & 206 & 228 & 218 & 224 \\ 710 & 146 & 118 & 180 & 167 & 170 & 156 \\ 720 & 119 & 109 & 158 & 152 & 121 & 117\end{array}$

Table 1: Raw counts in the TOF spectra for neutrons from $\pi-p \rightarrow \eta n$

$\begin{array}{cllllll}\mathrm{P}(\mathrm{MeV} / \mathrm{c}) & \mathrm{A} 1 & \mathrm{~A} 2 & \mathrm{~A} 3 & \mathrm{~A} 5 & \mathrm{~A} 6 & \mathrm{~A} 7 \\ 670 & 12 . & -5 . & 9 . & -5 . & 3 . & -6 . \\ 675 & -23 . & 3 . & -19 . & -12 . & -4 . & 13 . \\ 680 & 10 . & 3 . & 10 . & 18 . & 1 . & -7 . \\ 685 & 28 . & 44 . & 51 . & 33 . & 20 . & 18 . \\ 690 & 59 . & 72 . & 81 . & 48 . & 61 . & 40 . \\ 695 & 60 . & 109 . & 97 . & 111 . & 86 . & 71 . \\ 700 & 20 . & 54 . & 84 . & 101 . & 109 . & 117 . \\ 710 & -14 . & 20 . & 58 . & 40 . & 61 . & 49 . \\ 720 & -41 . & 11 . & 36 . & 25 . & 12 . & 10 .\end{array}$

Table 2. Background corrected results obtained by subtracting the average yields below threshold (670-680 MeV/c).

$\begin{array}{cllllll}\mathrm{P}(\mathrm{MeV} / \mathrm{C}) & \mathrm{A} 1 & \mathrm{~A} 2 & \mathrm{A3} & \mathrm{A} 5 & \mathrm{A6} & \mathrm{A} 7 \\ 680 & 0 & 0 & 0 & 0 & 0 & 0 \\ 685 & 13 & 4 & 1 & 0 & 0 & 0 \\ 690 & 76 & 129 & 136 & 91 & 29 & 1 \\ 695 & 40 & 60 & 72 & 104 & 133 & 107 \\ 700 & 31 & 47 & 54 & 65 & 70 & 77 \\ 705 & 27 & 40 & 46 & 54 & 54 & 53 \\ 710 & 24 & 36 & 40 & 47 & 48 & 44 \\ 715 & 22 & 34 & 38 & 44 & 44 & 41 \\ 720 & 20 & 31 & 36 & 41 & 39 & 38\end{array}$

Table 3. Normalized yields from the Monte-Carlo calculation 

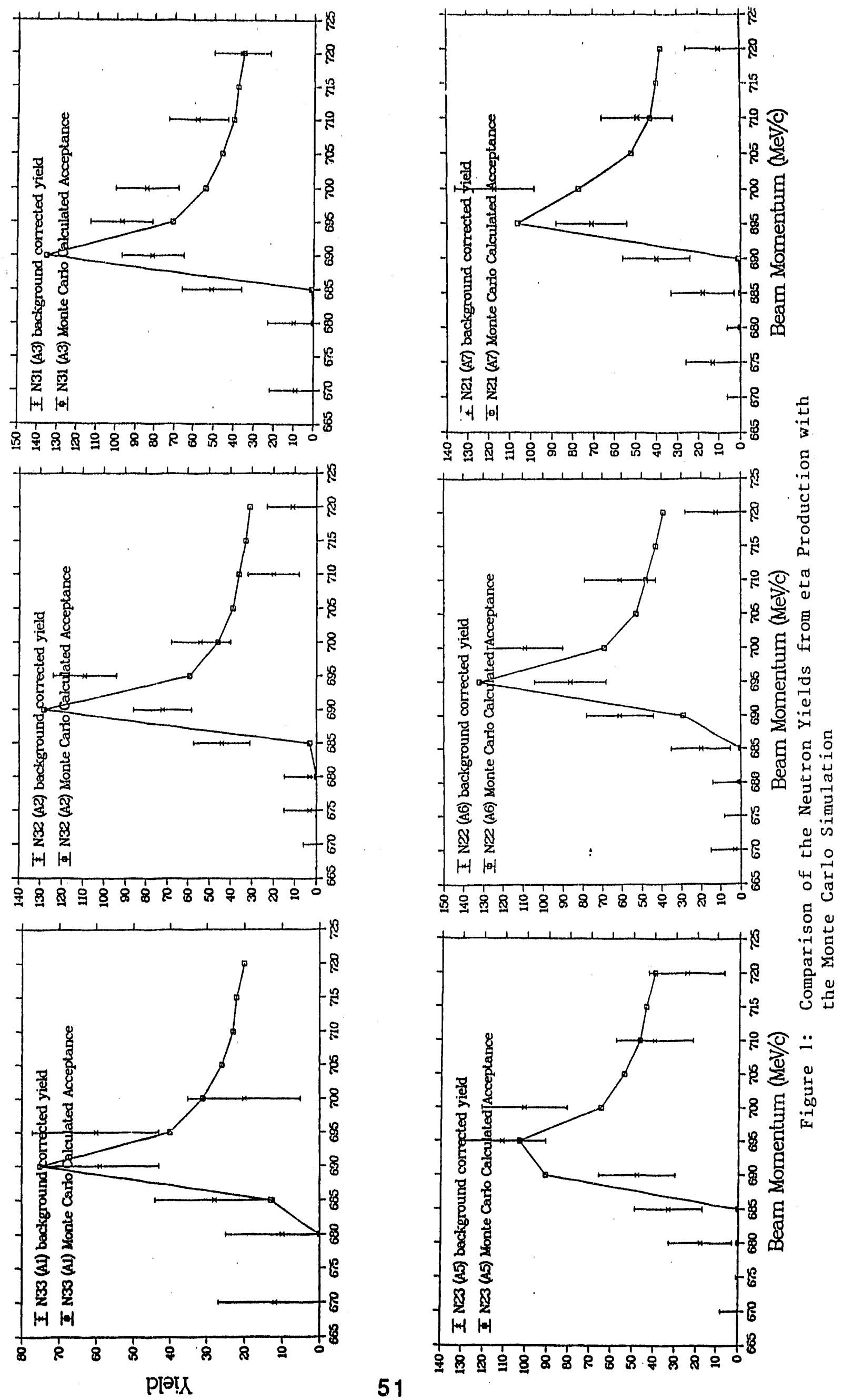


\section{Detector Tomography Facllity}

Tyson Browning and Kelth Elmore contributed to this section.

A computed tomography facility has been built and is currently in use at Los Alamos National Laboratory. When testing of the crystals for the Neutral Meson Spectrometer (NMS) is finished, the investigators plan to move the apparatus and to re-assemble it in the newly completed nuclear and particle physics laboratory at Abilene Christian University. The laboratory is part of a renovation of the Foster Sclence Building which was completed in early January of this year. The tomography facility incorporates arrays of scintillators and wire chambers to tag cosmic muons that pass through a detector under test. By recording the detector signals from the random trajectories of cosmic rays and obtaining adequate counting statistics, a threedimensional response of the detector crystal can be obtained using Fourier transform techniques. The detector response depends on the light collection and uniformity of the crystal.

Scintillation detectors made of sodium lodide (Nal), cesium lodide (Csl), bismuth germanate (BGO) and other materials are used to measure the energies of particles in modern experiments. Charged particles traverse the crystal, depositing energy through ionization losses. Gamma rays are detected by a multistep process in which the gamma ray initiates an electromagnetic shower. A high-energy gamma ray produces an electron-positron pair, which subsequently undergoes Bremsstrahlung scattering yielding a lower-energy gamma ray, which in turn undergoes pair production, etc., until the energy is dissipated. For both charged particles and gamma rays, the light that is produced as the atoms de-excite is proportional to the energy deposited. An electronic signal which is in turn proportional to the light collected at a photocathode is produced by a photomultiplier assembly which views the crystal.

The objective of this project is to determine the performance of individual scintillation detectors before including them in a large, multi-detector array such as the NMS. The key factor in the performance is simply the amount of light that reaches the photomultiplier tube as a function of the location in the detector where the energy of a particle is deposited. For example, if one region of a detector produces $10 \%$ more light than another region then the resolution is limited to this level even if the statistics (determined by the number of photoelectrons produced at the photocathode) would indicate that a much better resolution, typically on the order of a few percent, could be obtained. Geometric factors such as the size of the crystal and of the photomultiplier tube are of prime importance. Another factor is the nonuniformity induced by the crystal growth method. 
The tomography concept is probably famillar to the reader in the context of Computer-Aided Tomography (CAT) obtained by passing a collimated $x$-ray beam through an object (or a patient) from varlous directions and then deconvoluting the transmission information to obtain the density distribution of the materlal. Threedimensional information ranging from cracks in welds to tumors in patlents is obtained. The detector tomography concept described here is similar, except that information on the detector response is obtained for particles traversing the active volume. A schematic is shown in Fig. 1. The apparatus is triggered by a two-fold coincidence between elther of the two scintillators and any one of the crystals being tested, indicating the presence of a cosmic muon. The trajectory of the muon both before and after passing through a test crystal is obtained from an $x-y$ wire chamber above and below the crystal, respectively. If additional precision is desired, a second wire chamber could be added so that there are two chambers both above and below the crystals. The random nature of the incident cosmic rays insures that the crystals are uniformly illuminated along a large number of trajectories. The solld-angle acceptance of the apparatus is such that all portions of the crystal can be tested without having to rotate elther the apparatus or the crystal, thus ellminating the problem of accurately determining the position of the crystal a second time. However, rotation of the crystal would allow one to check the systematic accuracy of a scan. Such a tomography system is discussed in Ref. 1, the primary difference being that scintillation counter hodoscopes (small strips of scintillator to give position information) were used instead of drift chambers. The present system will give much better position resolution $(\sim 200 \mu \mathrm{m})$ of the muon trajectories than that afforded by the hodoscopes of Ref. $1(3.8 \mathrm{~cm})$.

The computer analysis of the experimental data will consist of four separate tasks. The first task is the calculation of the energy deposition per unit length for an ideal crystal. The balance of the analysis consists then of calculating the uniformity of the departure of the actual crystal from this ideal crystal behavior. The second task is a Monte Carlo calculation of the energy deposition spectra for the possible path lengths muons may follow within the crystal. The third task consists of the calculation of the departures from the ideal by comparing the ideal behavior and the reconstructed shifts. The result of this intermediate step is a deviation from uniformity, for each unit volume of the crystal, expressed in $\mathrm{MeV} / \mathrm{cm}$. Obviously, a uniform result of zero deviation would be optimal, but in reality the degree of uniformity is more important than the amount of deviation. The fourth task is the production of a second image which indicates the accuracy of the reconstruction process by comparing the summed deviations and the Monte Carlo fitted energy spectra shifts. The analysis actually 
prepares a collection of two-dimensional slices of the crystal and then stacks them to produce the three-dimensional deviation per unit volume values discussed above.

The positional resolution of $200 \mu \mathrm{m}$ mentioned above will result in the possibility of analyzing unit volumes on the order of a cublc centimeter. Further, the angle of acceptance and the ability of analyzing muon trajectories having significant departures from the normal will allow this apparatus to make use of more of the incident muons. We belleve that, given this degree of granularity, the final resolution of this apparatus will be limited not by the ability to locate and describe nonuniformity in the response but by the cosmic muon flux per unit volume. The final statistics and analysis of crystal response should be improved by orders of magnitude over the results of Ref. 1.

Work on the apparatus began during the summer of 1990 when four wire chambers (two with a single pair of $x-y$ planes and two with double $x-y$ planes) were constructed in Los Alamos by ACU students Scott Garner and Jason Phillips. These chambers, described in Ref. 2, are of the design used at EPICS and HRS at LAMPF. That fall, a used Microvax II computer was purchased for data acquisition and was subsequently taken to Los Alamos. At that time, a request was made to begin construction of the scintillators and light guides for the apparatus. In May 1991, two students (Tyson Browning and Keith Elmore) returned to Los Alamos to work full time on the project. By that time, a light tight box had been designed and was being built. While waiting for the box to be completed, the students attached the phototubes to the scintillators and then mounted the scintillators so that they would remain straight and be easy to move. They then measured and made all of the desired cables running from the apparatus to the electronics racks. Once the box was made; the whole setup was assembled and wired in the Biomed control room which had been obtained for the experiment. (A diagram of the electronics is included as Fig. 2.) Work then began to plateau the wire chambers and scintillators to find their optimum working voltage.

During the first week of July the first set of five crystals for the NMS was received from Horiba, and data collection began. An additional five were received toward the end of that month, but since that time none of the remaining crystals have been shipped. While data were being collected on the first crystals, some initial software development was done. The first step of finding the energy deposition per unit path length required a program to calculate the path length through any portion of a crystal. After some fairly complicated geometry work needed to handle the tapered crystals being used, Browning wrote software to handle this first task. Since the beginning of the Fall semester, additional software work has been started to handle the remaining three tasks involved in the data analysis. 
The investigators are eagerly anticipating the incorporation of the tomography apparatus into the nuclear and particle physics laboratory at ACU because of the exciting prospects for present and future students of the university. They can learn how to operate a computer-based data acquisition, learn the principles of operation of wire chambers, participate in data analysis, etc., on an in-house experiment. In the past, most of these activities were confined to summer research at either LAMPF or Fermilab. Work has already been done to ready the facility with some of the materials that can not be moved from Los Alamos. Funds from the ACU Research Council are being used to build the basics of the gas handling system and to purchase some electronics. Along these lines, it is planned to have students build simple drift cells so that ihey can study the effects of different gas mixtures and additives on wire chamber behavior. The investigators feel that it is an exciting project from the physics perspective and will make major contributions to the development of future detector systems. The facility should be in demand for years to come.

\section{References:}

1. D. H. Dowell, A. M. Sandorfi, A. Q. R. Baron, O. C. Kistner, G. Matone, C. E. Thorn and R. M. Sealock, Nucl. Inst. and Meth. A286, 183 (1990). This article describes the only known application of computed detector tomography with cosmic muons. The wire chambers ured to trigger data collection in the proposed apparatus will provide a more precise determination of the muon trajectories.

2. L. G. Atencio, J. F. Amann, R. L. Boudrie and C. L. Morris, Nucl. Inst. and Meth.183, (1990). The drift chambers to be used for this project are of the design described in this article.

3. D. Renker, Proceedings: ECFA Study Week on Instrumentation Technology for High-Luminosity Hadron Colliders, 14-21 Sept. 1989 (CERN 89-10), p. 601.

4. C. M. Rozsa, et at, ibid, p. 599. 


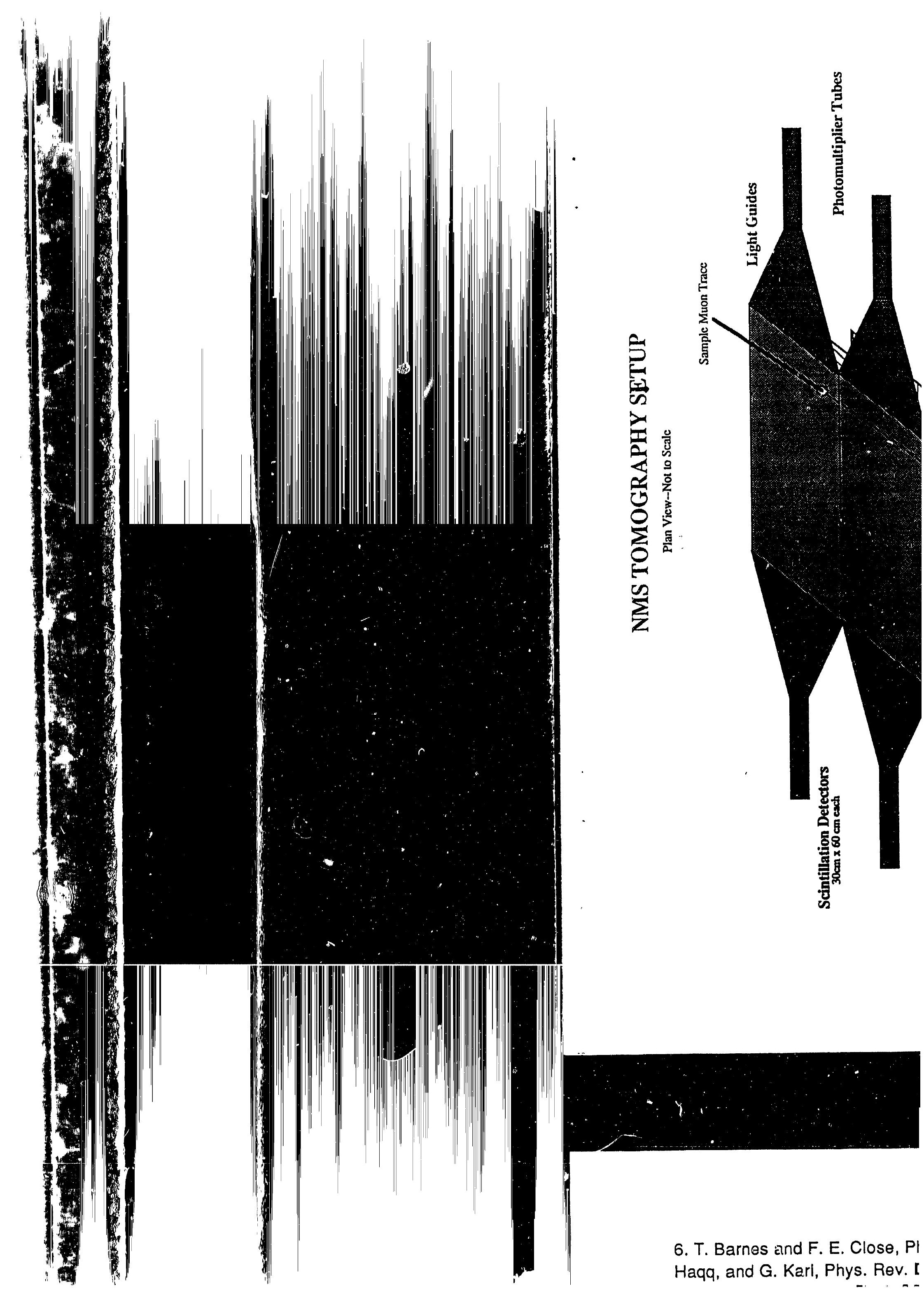




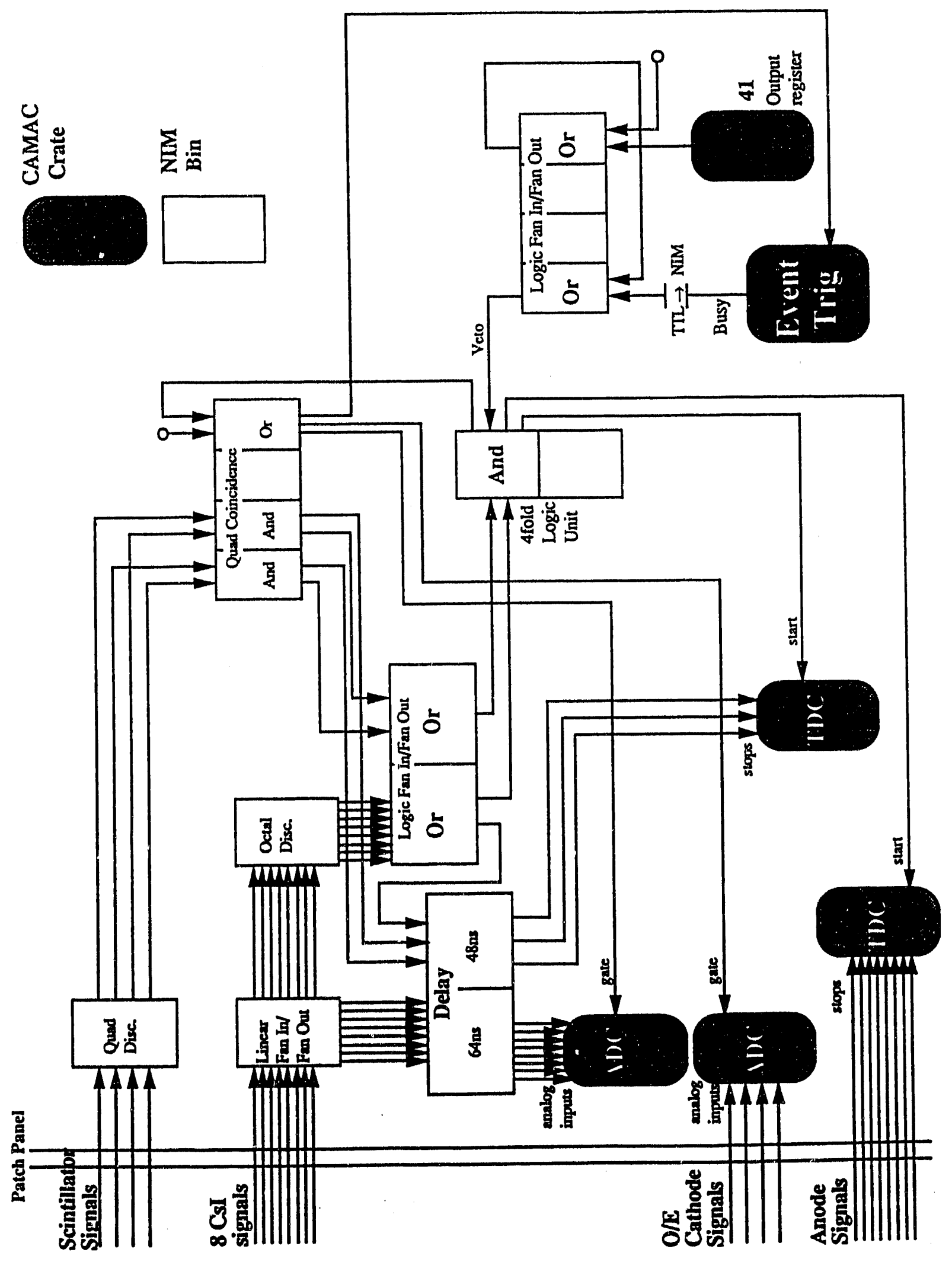

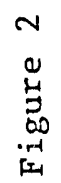




\section{FNAL E789: Nuclear Dependence of Charm and Beauty Quark Production and a Study of Two-Prong Decays of Neutral D and B Mesons}

\section{A. E789: Introduction}

Abilene Christian University has been a collaborator since October, 1988 on Fermilab E789, a fixed target experiment. This collaboration consists of P2 Division of the Los Alamos National Laboratory, Fermilab, Northern Illinois University, University of Chicago, University of California at Berkeley, University of South Carolina, Academia Sinica (Taiwan), and Abilene Christian University. The experiment is using the E605/E772 spectrometer located at the Meson East experimental hall at FNAL.

The experiment has two aspects. One is to measure the nuclear dependence of the production of charm and beauty quarks. The other is to study two-prong decays of $D$ and $B$ mesons with particular interest in the charmless decays of the latter. This experiment was approved in October 1988 and began its test run in the summer of 1990. After the extended shutdown at Fermilab, data collection was resumed in August 1991 and was completed in January 1992. E789 was approximately one month later in its startup than other FNAL experiments due to safety studies needed for the high rate conditions requested.

Roughly 1.5E9 events were written to $15008 \mathrm{~mm}$ tapes. The beam time was divided roughly equally between charm and beauty running conditions. Analysis of these data is currently underway at FNAL, LANL, and LBL. It is estimated that roughly 1000 MIP-years are needed for the initial event reconstruction. Present computing resources at the three institutions mentioned should be able to allow the first pass analysis to be completed within the next 10-18 months.

\section{B. E789: Physics goals}

The goals of E789 are to measure rare two-prong decays of the neutral $B$ and D mesons and to measure the nuclear dependence of charm (c) quark and beauty (b) quark production. The $B$ meson study is motivated by the observation of a large amount of mixing in the $B^{\circ} \overline{B^{\circ}}$ meson system and that the amplitude for $b \rightarrow u$ conversion might be large.1 The $D$ meson studies come essentially for free because the requirements for this work are a simplified version of that for the $B$ mesons. The observation of charmless $B \rightarrow h+h$ - (dihadron) decays would contribute to the determination of the term in the Cabibbo-Kobayashi-Maskawa matrix, $V_{u b}$. Such two-prong decays have not yet been observed. One can find many papers on heavy quark physics and two papers of F. Gilman2 have extensive reference sections that 
indicate the breadth of the interest in this field. The nuclear dependence measurements are partially motivated by the success of FNAL E772, which used the same spectrometer, and has generated considerable interest in nuclear physics.

E772 yielded the first high-precision A-dependence of Drell-Yan (DY) production. 3 in addition, it has produced the first measurements of the A-dependence of $\psi^{\prime}$ and $Y$ (upsilon) production.

Also, E789 will search for several expected b-quark states such as $B_{d}, B_{s}$, and $\Lambda_{b}$. Simultaneously with the proposed search for $B_{d}, B_{s}, \Lambda_{b} \rightarrow h+h-(e . g, \pi+\pi-, K+K-p \bar{p}$, $\pi^{ \pm} K^{\mp}$ for meson decay, $\mathrm{p} \pi-\overline{\mathrm{p}} \pi^{+}, \mathrm{pK}, \overline{\mathrm{p}} \mathrm{K}^{+}$for baryon decay), the experiment will be sensitive to other b-quark decays. Sensitivity will exist for $B_{d}, B_{s} \rightarrow \mu \mu, e \mu, \theta e ; B \rightarrow J / \psi$, $\psi^{\prime}, \chi_{0} ;$ and $\eta_{b}, Y_{,} \chi_{b} \rightarrow h+h$. Information will be obtained on the mass, lifetime, and production dynamics of any state detected.

A list of some of the specific physics goals of this ambitious experiment are the following:

1) to extend the range in Feynman-x over which the nuclear dependence of $J / \psi$ and $\psi^{\prime}$ production cross sections have been measured,

2) to study A-dependence of open charm production $\left(\mathrm{J} / \psi\right.$ and $\psi^{\prime}$ data measure hidden charm, $D$ meson data will measure open charm),

3) to study $D \rightarrow \pi K$ decays,

4) to study dileptonic $D$ decay modes at the 10.7 branching ratio levels,

5) to produce the $\eta_{c}$ and $\chi_{c}$ resonances of the charmonium system,

6) to determine the values (or upper limits) of the branching ratios for a variety of b-particle dihadronic decays and thus help determine $V_{u b}$ of the CKM matrix,

7) to search for $B_{s}$ and $\Lambda_{b}$,

8) to measure the lifetimes and masses of $B_{d}, B_{s}$, and $\Lambda_{b}$,

9) to measure the $B \rightarrow J / \psi X, \chi^{0} X$, and $\psi^{\prime} X$ inclusive decays,

10) to search for exclusive dilepton decays $B \rightarrow \mu^{+} \mu^{-}, \theta^{++} \theta^{-}, \mu+\theta^{-}, \mu \cdot \theta^{+}$,

11) to study inclusive dilepton decays of $b \bar{b}$ pairs, and

12) to search for the $\eta_{b}$ and for dihadron decays of $Y$ and $\chi_{b}$ states.

The expansion of the physics goals of E789, which initially was to search for charmless beauty decays, was prompted, in part, by the increased interest in heavy quark production in relativistic heavy-ion collisions from CERN NA38, and by new results from Fermilab E772 on the A-dependence of $\mathrm{J} / \psi, \psi^{\prime}$, and $\mathrm{Y}$ production. Many theoretical models now attempt to describe the heavy-ion data as well as data from hadron-nucleus collisions (including older data from CERN NA3 and Fermilab E537) in a unified way. Taken together, the new experimental results and the theoretical 
interest which they have inspired, strongly suggest additional nuclear dependence measurements relating to heavy quark production and propagation.

The spectrometer being used is the old E605 spectrometer4 shown in Fig. 1, which is well known for its high resolution $\mu+\mu$-studies of the $Y$ (upsilon) region. This same spectrometer was used in E772 to measure the nuclear dependence of $\mathrm{J} / \psi$ and $\psi^{\prime}$ production. This spectrometer is roughly 60 meters in length. There are two analyzing magnets, SM12 and SM3 which define the optics of the spectrometer. There is an internal beam dump inside SM12 which is the larger magnet immediately downstream of the target region. Between SM12 and SM3 is a set of drift chambers (Station 1) and an $x-y$ hodoscope array ( $X 1$ and $Y 1$; coordinate system is $Z$ in beam direction, $+x$ is beam left and $+y$ is up in the lab). Following SM3 is a hodoscope array (Y2) and another set of drift chambers (Station 2). Next is the Ring Imaging Cherenkov $(\mathrm{RICH})$ detector which consists of a 15 meter gas radiator volume with an array of mirrors at the end to reflect and focus the vacuum ultraviolet (VUV) Cherenkov light on two multistep avalanche chambers located on each side of the RICH. Following the $\mathrm{RICH}$ is a third set of drift chambers (Station 3 ), a third hodoscope array ( $X 3$ and $Y 3$ ), an electron calorimeter, a hadron calorimeter, a fourth hodoscope array (X4 and Y4), a thick absorber, and a set of prompt tubes for the detection of muons.

The modifications to the E605/E772 setup have included a new set of high rate drift chambers for Station 1, a microstrip vertex detector, changes in the aperture of BM12 to allow running at the low field values required for E789, and the modification of the hodoscope arrays at Stations 1, 2, and 3 (the latter project was carried out by $A C U)$.

An important part of this spectrometer is the $\mathrm{RICH}$ detector. This was one of the first large RICH detectors built and has been described in several articles 6 . Combining its information with that of the electron and hadron calorimeters and the muon detectors, this experiment will have good $\pi / \mathrm{K} / \mathrm{p} / \mathrm{e} / \mu$ separation. This is essential for the study of several of the decay channels discussed above. The RICH detector itself has not been modified, but the ADC's of the readout chain have been replaced with LeCroy 1885 FASTBUS ADC's. ACU took a significant role in the commissioning of the new data acquisition electronics and in getting this detector back on line (it had not been used for the last several years).

Hadronic production of heavy quarks ( $c$ and $b$ ) from nuclear targets holds much interest for nuclear physics. At high energies ( $\geq 100 \mathrm{GeV}$ ) the dominant production diagram is gluon fusion, $g g \rightarrow q \bar{q}$. Hence nuclear effects on the gluon structure function may be observable in measurements of the A dependence of charm and beauty production. The quarkonium resonances, $J / \psi$ and $Y$, have easily 
detectable experimental signals and can be used to analyze the dynamics of reaction processes.

\section{Future of E789 Collaboration and Summary}

Depending on the analysis of current data, an additional year is expected to be requested for the next fixed target run at Fermilab (expected sometime in 1993-4 if the FNAL fixed target program is continued). Due to the delayed startup of E789 because of safety issues, over one month of running was lost. Newly imposed radiation limits in the Fermilab experimental areas also forced E789 to run at roughly one-half of the requested intensity. In spite of these problems, it appears at this time that E789 will still be able to set the world's best upper limits of $D$ to dilepton decays ( 0.5E-5 should be possible). The limits for $B$ decays into dileptons and dihadrons will not likely fare as well, with the best limits only being around $1 \mathrm{E}-4$. Thus, some of the goals stated in Section $B$ above will depend on further running. At this time, it is difficult to make any definite comments along these lines. Considering that the past fixed target run was completed over 14 months late from schedules given in July of 1990, making any plans at this stage on a future fixed target run will almost definitely be offl

During the next year, ACU will concentrate on fine tuning the $\mathrm{RICH}$ analysis part of the event reconstruction. This is not done in the initial passes through the data and is a project that should be able to be conducted easily on the two DECstation $5000 / 200$ computers being provided by the Academic Computing division of ACU. During the past year an $8 \mathrm{~mm}$ tape drive was purchased for one of the DECstations with this grant's funds so that the RICH analysis could be pursued on site at $A C U$.

Since the work at ACU depends on the attraction of high quality undergraduates, the mixture of research programs at both FNAL and LAMPF have been helpful in recruiting very good students for ACU's research program. This work has also expanded the opportunities of the undergraduate students and helps them to see additional possibilities for careers in particle physics research. It is worthwhile to note that from the last two years' undergraduate students, three are in graduate programs in particle physics.

One example of this student assistance was work performed by Randy Schnathorst during the summer of 1991 (he was actually supported by P2 division of LANL after being supported by this contract in the summers of 1989 and 1990). $\mathrm{He}$ worked on calibrating the gains of the individual calorimeter channels and the timing of the silicon vertex detector channels. His involvement in data analysis has continued during the academic year where he is helping install the analysis code on a DECstation. He also modified the data analysis software for the Ring Imaging 
Cerenkov Detector (RICH) to reflect the changes made necessary by the installation of a FASTBUS based DAQ system. Finally, he updated the technical documentation pertaining to the data formats used by the various detector systems. This 50-page document, called "The Last Word," contains every bit-level detail of the formats in which data are written to tape by the various detector systems. It is used as a reference by everyone working on the electronics or the software. Much of the electronics had been replaced by more advanced equipment in E789, making the details of the old data formats obsolete. The old documentation was handwritten; the new document was typeset in $\mathrm{TeX}$ to facilitate any later modifications that might be made.

\section{References:}

1. H. Albrecht et al., Phys. Lett. B192 (1987) 245; C. Albajar et al., Phys. Lett. B186 (1987) 247; H. Band et al., Phys. Lett. B200 (1988) 221.

2. F. J. Gilman, SLAC-PUB-4736, Oct. 1988, Invited talk given at the Heavy Flavor Physics Symposium, Beijing, China, 10-20 Aug. 1988; F. J. Gilman, SLAC-PUB4955, April 1989, Invited lecture at the Third Mexican School of Particles and Fields, Cuernavaca, Mexico, 5-16 Dec. 1988.

3. D. Alde et al., Phys. Rev. Lett. 64 (1990) 2479.

4. Y.B. Hsiung et al., Phys. Rev. Lett. 55 (1985) 457; J.A. Crittenden et al., Phys.

Rev. D 342584 (1986)

5. T. Nakada (editor), Feasibility Study for a $B$ Meson Factory in the CERN ISR

Tunnel, CERN 90-12, 30 Mar. 1990; (also see Proposal for an Electron

Positron Collider for Heavy Flavour Particle Physics and Synchrotron

Radiation, PSI-PR-88-09 on which the CERN proposal is based)

6. R. Bouclier et al., Nucl. Instr. and Meth. 205 (1983) 403; P. Mangeot et al., Nucl. Instr. and Meth. 216 (1983) 79; H. Glass et al., IEEE Trans. on Nucl. Sci. NS-30 (1983) 30; M. Adams et al., Nucl. Instr. and Meth. 217 (1983) 237. 


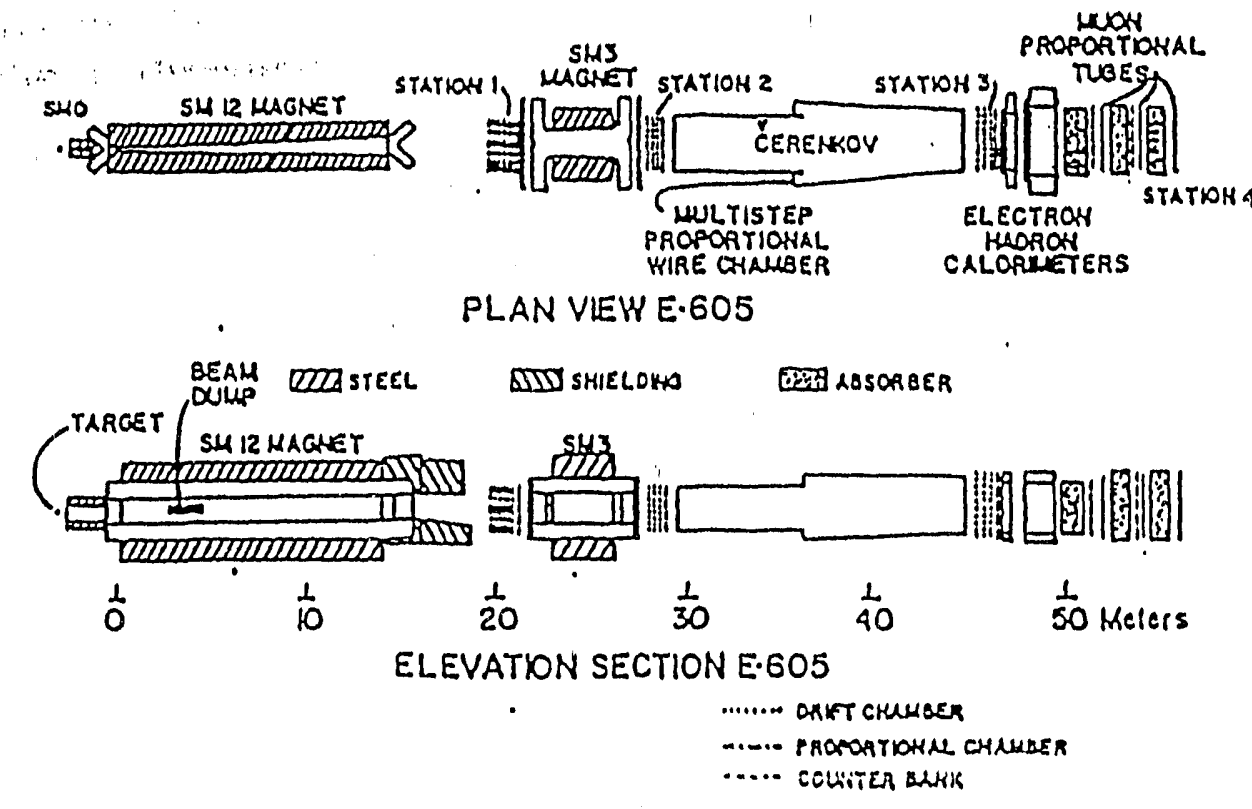

Figure 1

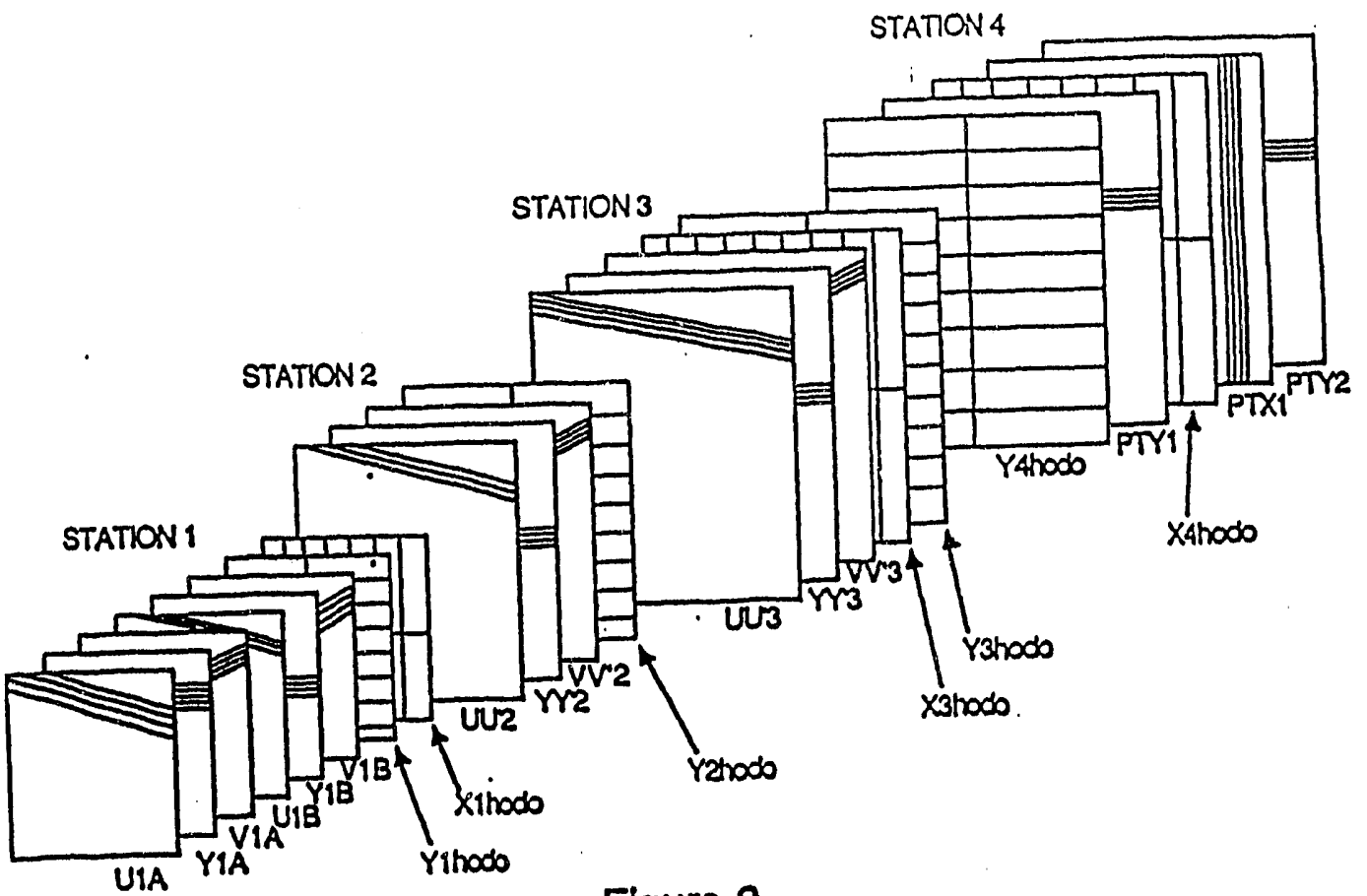

Figure 2 


\section{V. $\mathrm{N}^{*}$ Collaboration at CEBAF}

$A C U$ is a member of the $N^{*}$ collaboration at CEBAF, which plans to utilize the CEBAF Large Aperture Spectrometer (CLAS). Participation in this collaboration so far has been limited to attendance at a few of the collaboration meetings. Sadler attended the $\mathrm{N}^{*}$ workshop at CEBAF in June 1991. Among the proposals that have been approved are:

- Ele troproduction of the $\Delta$ (1232) Resonance, V. Burkert (CEBAF) and R. Minehart (Virginia), spokespersons,

- Measurement of Polarized Structure Functions in Inelastic Electron Proton Scattering using the CLAS, V. Burkert, spokesperson,

- Measurement of $p\left(e, e^{\prime} \pi^{+}\right) n, p\left(e, e^{\prime} p\right) \pi^{\circ}$, and $n\left(e, e^{\prime} \pi\right) p$ in the $2 n d$ and 3rd Resonance Regions, Minehart, Burkert and M. Gai (Yale), spokespersons, - A Study of the $S_{11}(1535)$ and $P_{11}(1710)$ in $p\left(e, e^{\prime} p\right) \eta$. S. Dytman (Pittsburgh) and K. Giovanetti (James Madison), spokespersons, and

- A Measurement of the Electron Asymmetry in $p\left(e, e^{\prime} p\right) \pi^{\circ}$ and $p\left(e, e^{\prime} \pi^{+}\right) n$ in the Mass Region of the $P_{33}$ (1232), Burkert and Minehart, spokespersons.

There are several ways in which ACU may be able to help in the construction of the CLAS detector, but the involvement to date has been minimal due to our commitments at LAMPF and Fermilab. Experience gained while working on FNAL E789 and on the LAMPF NMS should allow the ACU program to assist in some areas such as scintillators or electronics. The tomography apparatus discussed previously in this progress report may be useful at some time to make some studies related to the CLAS detector.

The physics is closely related to the research that ACU has been doing for the past decade. Discussions have been held with some the spokespersons for the experiments (V. Burkert of CEBAF, R. Minehart of Virginia, S. Dytman of Pittsburgh and $P$. Stoler of Rensselaer) regarding ACU's current commitments and the possibilities for future participation. It was generally felt that serious involvement starting in 1992 or 1993 would be acceptable, after the conclusion of the data taking for E789 at Fermilab. Burkert has volunteered to help supervise students, so it should be possible for ACU to send two students during the summer of 1993 even if experiments on the LAMPF NMS are taking up a considerable part of the principal investigators' time.

There are several good student candidates for this work that are just starting their undergraduate work at ACU. Such a student would be able to participate the next three or four summers, allowing him to be more useful as the CLAS nears completion. This approach has worked out very well in the case of Derek Lane, Steve LOe, and Randy Schnathorst who were all involved in the research program at ACU 
after their first year of undergraduate work. Lane is currently a graduate student at lowa State University in High Energy Physics, Loe is now a graduate student at University of Pennsylvania in nuclear physics, and Schnathorst is planning on pursuling a Ph.D in particle physics after graduation this year.

While the CEBAF program is probably not far enough along for ACU to send a thesis student for the next year or two," by the end of the period of this renewal (199394), this will certainly not be the case. One of the students who worked on the SPNPI experiment has expressed interest in enrolling in the special program ACU has with Texas Tech University and would be a good candidate to work on this program if sufficient funds are available. For ACU to take an active role in the CEBAF work, the investigators belleve that it is essential to have a representative at CEBAF full time by the time the above experiments are getting ready to run. The computing facilities at ACU are excellent and will facilitate the data analysis for such a graduate student. It is not necessary to be present on the ACU campus to utillze these facilities as ACU is on several of the high speed computer networks. The students could spend most of their time at CEBAF where they would be of the most use to the program, only returning to ACU when their thesis/dissertation is nearing its final form and they need closer contact with the principal investigators.

"Note that since ACU works its graduate program by cooperative agreements with Texas Tech University and Rudjer Boskovic Institute, these students will normally be advanced students that have finish most, or in the case of the RBI students, all of their coursework. So these students will be ready to begin their thesis research when they are funded by ACU and will need to work on a running experiment. Thus, the lack of graduate courses at ACU is completely irrelevant for these students. 


\section{PILAC Involvement}

ACU involvement during the past year in the proposal to build a Plon Linear Accelerator (PILAC) at LAMPF has been extensive. Sadler, with W. R. Gibbs (formerly at T division at LANL, presently at New Mexico State) co-authored the sectlon "Baryon Resonances at PILAC" for the final report, PILAC User's Group Report on Physics with PILAC, October 15, 1991. Jeff Arrington worked on the design of the superconducting quadrupoles in collaboration with Arch Thiessen, Group MP-14 at LAMPF. Reports of each of these efforts are included below.

\section{A. Overview of Physics Goals}

Other contributors to report on baryon resonances included J. A. Carlson, J. F. Friar, B. F. Gibson, J. N. Ginocchlo, T. Goldman, L. Heller, P. Herczeg, A. Leviatan, M. Mattis, R. R. Silbar (Los Alamos National Laboratory), G. Glass (Texas A\&M University), A. Kaidalov (Institute of Theoretical and Experimental Physics, USSR), E. R. Kinney (University of Colorado), L. Kisslinger (Carnegie Mellon University), E. Lomon (Massachusetts Institute of Technology), N. Mukhopadhyay (Rensselaer Polytechnic Institute) and I. Strakovsky (St. Petersburg Nuclear Physics Institute). The following discussion is condensed from that report.

Understanding the strong interaction is a major goal of nuclear and particle physics. The study of the structure of baryons in terms of the underlying quark and gluon degrees of freedom of QCD, now the best candidate for the theory of strong interactions, provides valuable tests for this theory. In the low-energy region to be explored by PILAC the development of QCD non-perturbative methods for treating the structure of mesons and baryons is a fundamental problem. It is essential to develop satisfactory models of baryons in this region of soft QCD in order to be able to treat the transition to perturbative QCD at high momentum transfer, where one can use methods such as those developed for QED.

An extensive new program of experimental study of baryon resonances, excited states of nucleons, is needed in order to realize these goals. It was the rich spectrum of baryon resonances and mesons which led to the development of quark models of hadrons [see Ref. 1-5 for reviews]. Some of these models have been quite successful in describing the spectrum (and many properties of the hadrons) in terms of constituent quarks. The relation of these models to QCD, however, has not been established.

Previous experimental and theoretical research results suggest some specific experimental goals which could lead to remarkable developments in the study of how 
QCD is reallzed in nature. One crucial aspect of excited nucleon states is the role of gluonic degrees of freedom. At present there is no compelling experimental evidence that gluonic modes must be included in order to account for the excitation of any baryon resonance; yet there are compelling theoretical arguments that the excitation of gluonic modes should occur. Because of the difflculty in developing models based directly on QCD in the soft region, guidance from improved experiments is necessary.

There have been two approaches for introducing nonperturbative QCD effects starting from the QCD Lagranglan itself for the study of baryons. Flrst, lattice gauge calculations have given valuable information about gluonic states (quarkless states called "glueballs"), as well as information about confining potentials. Although there are estimates of the nucleon mass and some properties of the nucleon, the method has not been developed sufficiently to give detailed models of the nucleon or baryon resonances.

The method of QCD sum rules is a second method for treating nonperturbative aspects of QCD. With this method one has been able to relate properties of baryons to condensates, vacuum expectation values of certain operators that appear in the shortdistance expansion of QCD. Hybrid states, states with explicit (valence) gluonic components, have been predicted using these methods. This approach is promising for the study of specific QCD effects in hadrons.

There have also been phenomenological models of hybrid baryon resonances, which are valuable in providing some guidance for experiments. Proposed experiments on baryon resonance electroproduction that seem most promising for providing evidence of gluonic degrees of freedom are motivated in part by such models. All of the known baryon resonances have been discovered via hadronic probes; however, a new era for the study of baryon resonances is beginning in the 1990 's with the development of high-duty-factor electron accelerators. In particular, the CEBAF accelerator will have sufficient energy to produce all of the $N$ and $\Delta$ resonances in the Particle Properties Summary Table. All of the baryon resonances that will be studied in the set of experiments with accepted proposals can be studled by PILAC experiments. In order to make full use of the results of the CEBAF baryon resonance electroexcitation experiments, it is essential that improved plon excitation experimental data be available. It is expected that the CEBAF program will start in 1994, so there is some urgency in developing the complementary PILAC program.

For the success of the complete overall program it would be vital that a second stage of PILAC be implemented for study of the higher resonances, where gluonic modes must be found if our current understanding of QCD is at all correct.

In this overview, experiments on pionic excitation of the first three regions of baryon resonances are discussed with emphasis on relationships with the accepted 
experiments at CEBAF. Note that in electroproduction experiments one is mainly interested in extracting the helicity $1 / 2$ and $3 / 2$ multipole photon-nucleon-resonance amplitudes.

For the first baryon resonance, the $\Delta(1232)$, an interesting set of studies will be carried out at CEBAF to determine the lowest electric and scalar multipoles in a transition which is dominated by the magnetic multipole. This can give valuable information about quark configurations of the $\Delta(1232)$. The main QCD information expected concerns the effective quark-quark interaction. This region has been very well explored by the present pion machines and it does not seem that improved pion experiments would be very important here.

In the second resonance region there are a number of specific problems for which PILAC experiments would be important. New experimental studies of the $P_{11}(1440)$ [the Roper resonance] and the $S_{11}(1535)$ are motlvated by specific questions related to $Q C D$. Other important questions which motivate new experiments in this region of $1.4-1.6-\mathrm{GeV}$ resonances are discussed in the following paragraphs.

Experimental studies of the $P_{11}(1440)$ resonance are strongly motivated by the observation that the lowest hybrid baryon (a qqqg configuration) in bag models 6 is expected to be a $P_{11}$ resonance. There has been a great deal of controversy about the analysis of this broad resonance (see Section II below). If the conventional picture of the Roper resonance as a radial excitation of the nucleon is correct, this resonance is mainly excited by longitudinal transition amplitudes. In the hybrid model,6 however, the longitudinal $\gamma-\mathrm{N}-\mathrm{N}^{*}$ transition amplitude is greatly suppressed.7 In the analysis of CEBAF experiments to extract the gamma transition amplitudes, the $\pi \mathrm{NN}^{*}$ vertex must also be included, since these are pion electroproduction experiments. A detailed pion excitation experiment is necessary for a satisfactory analysis of the Roper. This could provide an excellent opportunity for an important discovery in hadronic physics with critical implications for QCD-based models of hadrons.

The world's first complete data sets for $\pi \mathrm{N}$ scattering $8-14$ have been obtained at LAMPF in the region up to the Roper resonance. Higher energies are needed to complete this task. The Roper amplitudes display an unusual Q2 dependence which could be investigated with higher energy studies.

It should be noted, however, that relativistic corrections 15 could change the quark model predictions for the longitudinal transition amplitude. This is reminiscent of the difficulty in obtaining a clean signal for meson exchange currents or quark cluster effects in nuclear structure physics. Therefore, the study of the $P_{11}(1440)$ must be considered to be part of a more general program of study of baryon resonance excitations for gluonic excitations. It is an important part of that study in which PILAC will play a vital role. 
The need for extensive studles of the $S_{11}(1535)$ resonance is motivated by several results of previous studies. 16 The most important observation is that the $S_{11}(1535)$ resonance has a large branching ratio for decay into a nucleon and an eta. This feature is essential in the CEBAF study of this resonance. That study is motivated in part by the unusual $Q^{2}$ dependence of the amplitudes. Since the electroproduction amplitude of the $S_{11}(1535)$ is obtained from an analysis of the $\eta$ electroproduction amplitude, detailed information about $\pi-\eta$ production amplitudes at the $S_{11}(1535)$ resonance is essential for the success of the CEBAF project. This set of complementary experiments could then be used to examine the unusual momentum dependence to see if there is a characteristic QCD effect.

Another possible candidate for a hybrid baryon is the $P_{11}(1710)$ in the third resonance region. Arguments analogous to that given for the $P_{11}(1440)$ apply in this case. For this resonance $\pi-\eta$ production at PILAC is also essential for the irterpretation of the accepted CEBAF experiment on this resonance. PILAC experiments give specific information needed for the understanding of gluonic excitations.

A second generation accelerator would be needed to complete this study of baryon resonances. The capability of study of $2 \pi, \eta$, and other final states with pion excitation of $\mathrm{N}^{\text {"th }}$ s would make the PILAC facility invaluable in the search for gluonic excitations and for the development of QCD-based models. In addition to detailed information about known resonances, this facility could help complete the spectroscopy of baryon resonances. The answers to questions of states predicted in certain models and not seen in experiments and the detection of exotic states, states not predicted in constituent quark models, vill provide important information for the development of QCD-based theories. Moreover, it is crucial to study $\mathrm{N}^{*}$ excitations at high $Q^{2}$ (Ref. 17) in order to attempt to trace the evolution to the region of applicability of perturbative QCD.

\section{References}

1. A. J. G. Hey and R. L. Kelley, "Baryon Spectroscopy," Phys. Rep. 96, 71 (1983).

2. G. Karl, "Elements of Baryon Spectroscopy," Prog. Part. Nucl. Phys. 13, 243 (1984).

3. N. Isgur, "Nucleon Physics with Chromodynamics: From High Q2 to Baryon Spectroscopy to Nuclear Physics," Prog. Part. Nucl. Phys. 13, 275 (1984).

4. F. E. Close, "Gluonic Hadrons," Rep. Prog. Phys. 51, 833 (1988).

5. M. M. Giannini, “An Introduction to Nucleon Resonances," Prog. Part. Nucl. Phys. 24, 253 (1989). 
6. T. Barnes and F. E. Close, Phys. Lett. 123B, 89; 128B, 277 (1983); E. Galowich, E. Haqq, and G. Karl, Phys. Rev. D28, 160 (1983).

7. Z. Li, V. Burkert, and Z. Li, CEBAF-PR-91-032 (1991).

8. M. E. Sadler et al., Phys. Rev. D35, 2718 (1987).

9. F. O. Borcherding, UCLA thesis, 1982 (unpublished).

10. A. Mokhtari et al., Phys. Rev. D35, 810 (1987).

11. J. A. Wightman et al., Phys. Rev. D36, 3529 (1987).

12. C. J. Seftor et al., Phys. Rev. D39, 2457 (1989).

13. G. J. Kim et al., Phys. Rev. D41, 733 (1990).

14. D. B. Barlow et al., Phys. Rev. Lett. 62, 1009 (1989), and I. Supek et al., submitted to Phys. Rev. D.

15. M. Warns et al., Z. Phys. 45, 613; 627 (1990).

16. S. Dytman and K. Giovanetti, spokesmen, Proposal $3, \mathrm{~N}^{*}$ Collaboration at CEBAF. 17. P. Stoler, Phys. Rev. Lett. 66, 1003 (1991).

\section{B. Current Status and Needs}

PILAC, which would boost the attainable pion beam energy at LAMPF to 1-1.3 $\mathrm{GeV}$, would cover the low-lying $D_{13}$ and $S_{11}$ resonances and the cluster of resonances in the mass range of 1.6 -1.7 GeV. Here, six esiablished (3- or 4-star) isospin-1/2 resonances are degenerate when the uncertainties in their masses and widths are taken into account. Better $\pi$-p data (both elastic scattering and charge exchange) are sorely needed in this energy range in order to disentangle the struciure of these states. The situation is further clouded because questionable charge exchange data from the Rutherford Laboratory were heavily used by both the Karlsruhe-Helsinki and CMULBL efforts. These data apparently need to be shifted by $\sim 5 \%$ in momentum. Further, the backward-angle data for the analyzing powers lead to violations of isospin invariance by three standard deviations when combined with the recent accurate elastic data from the St. Petersburg and LAMPF groups. The LAMPF chargeexchange data are consistent with isospin invariance and do not agree with the Rutherford data at the back angles near $P_{\pi}=650-700 \mathrm{MeV} / \mathrm{c}$, where the measurements overlap.

Another cluster of six established resonances occurs near a mass of 1.9 $\mathrm{GeV} / \mathrm{c}^{2}$, but this time in the isospin-3/2 channel. Again, these states are essentially degenerate in their complex pole positions. If the one-star candidate for the $D_{33}$ is included, then all quantum numbers through the $F$ wave are exhausted. Is this an accident or is nature trying to tell us something? 


\section{Advantages of PILAC}

An obvious advantage that PILAC will have over pion beams at other facilities is the beam flux. PILAC fluxes of $10^{9} \pi+/ \mathrm{s}$ and $5 \cdot 10^{7} \pi / \mathrm{s}$ at $0.92 \mathrm{GeV}$ are not available at any other existing accelerator. The $\pi+$ flux at $0.92 \mathrm{GeV}$ will be more than an order of magnitude than that at BNL, even after the upgrade. At KEK, an extrapolation to 0.92 $\mathrm{GeV}$ from lower energies indicates that $1-2 \cdot 107 / \mathrm{s}$ for Either $\pi^{+}$or $\pi^{-}$would be available for pion-nucleon everiments.2

Since PILAC will serve as an if separator, the beam contamination will consist only of muons from pion decay in flight. The need to degrade a $\pi+$ beam in order to separate the protons using a bending magnet will be eliminated. The large proton contaminations for $\pi+$ beams above $600 \mathrm{MeV} / \mathrm{c}$ presently available at LAMPF limit the accuracy with which absolute measurements can be made. Pion beams at other facilities will not offer this advantage and, indeed, will have kaons that will have to be eliminated as well.

An additional unique feature that may be possible at PILAC is the ability to ramp the beam, 3 i.e., the ability to tune the beam at predefined momenta so that an excitation function of an observable could be obtained with a given experimental setup. If the detection apparatus were designed so that the acceptance was independent of the beam momentum, then the number of independent beam normalizations would be reduced to one.

The principle advantages PILAC offers in the measurement of inelastic channels are higher accuracy due to flux and resolution and beam purity. In particular, reactions such a $\pi \mathrm{N} \rightarrow \pi \pi \mathrm{N}$ and $\pi \cdot \mathrm{p} \rightarrow \eta n$ offer two kinds of advantages. The two pions can be expected to couple more strongly to higher Fock space states, and the $\eta$ helps by restricting isospin channels and coupling to gluon-rich states. These advantages, combined with the ability to choose (precisely) the minimum pion energy needed and the beam time available at a dedicated facility, afford the opportunity to establish the existence of many states, or alternatively, severe bounds $(\sim 1 \%)$ on the branching ratios to specific channels of a given or presumed state.

In addition to these production processes, elastic scattering and CEX are available tools. Good advantage can be taken of the latter (and of $\eta$ production) due to the availability of the high-resolution Neutral Meson Spectrometer (NMS). Finally, with the kinematics of produced states well constrained experimentally, one can even contemplate studying radiative decays, which have frequently provided excellent in sight into structural questions. 
As a specific example of all of the above, consider the study of the $\eta(1550)$. This is accessible at PILAC in $(\pi, \pi \eta)$ elastic, and $(\pi, \pi \pi)$ scattering. Because of copious production, its $\gamma$ decay to the $\Delta(1232)$ can also be studied.

Because of the reasonable total beam fluxes, there should be no difficulty in employing polarized targets to discern the quantum numbers of specific states. Particular examples in need of clarification are the one-star $N(1540)$ and $\Delta(1550)$ resonances which are required by collective models but are absent in the singleparticle quark model. The collective model also requires a second $\mathrm{N}(1440)$ and two $\Delta(1600)$ 's to complete the parity doublets. Do these states exist?

PILAC is a logical choice to perform a systematic measurement of observables in $\pi \mathrm{N}$ scattering in the resonance because of the infrastructure that exists. Specific examples include polarized targets (with both vertical and horizontal axes of polarization), polarimeters for measuring proton polarization, and the soon-to-becompleted neutral meson spectrometer (NMS) described in Sec. II.

\section{References}

1. A. Thiessen, "A Reference Design for PILAC", PILAC User's Group Report on the Physics with PILAC, Los Alamos, October 15, 1991.

2. M. leiri and R. Ransome, private communication.

3. A. Thiessen, private communication.

\section{Examples on Improvements PILAC Can Make on $\pi \mathrm{N}$ Observables}

Before embarking on a systematic program as described above, an appraisal is needed of what remains to be measured or needs to be remeasured. Examples of existing data in the interval $600 \leq \mathrm{P}_{\pi} \leq 1200 \mathrm{MeV} / \mathrm{c}$ are shown in Fig. 1-3. These plots are $d \sigma / d \Omega$ for $\pi \cdot p \rightarrow \pi \cdot p$ and $\pi+p \rightarrow \pi+p$ and $A_{N}$ for $\pi-p \rightarrow \pi \circ$, respectively. The partial wave analyses are plotted at $\theta_{\mathrm{cm}}=150^{\circ}$ and the data are shown in the interval $148^{\circ} \mathrm{s}$ $\theta_{\mathrm{cm}} \leq 152^{\circ}$. The data from this program (described in Sec. II), at $P_{\pi}=625,657$ and $687 \mathrm{MeV} / \mathrm{c}$, are difficult to pick out in Fig. 1 and 2 since the error bars are so small, but these points are indicative of the improvement that is possible if the PILAC facility is built. Fig. 3 shows the paucity of data for $\pi \mathrm{N}$ charge exchange and the poor agreement with existing PWA's. In general, the $\pi$-p data (both elastic and charge exchange) are in worse shape that the $\pi+p$ data.

No data for the spin rotation parameters exist between 0.7 and $6 \mathrm{GeV} / \mathrm{c}$. (laboratory pion momentum). This very important momentum interval was covered 
"once over lightly" when it belonged to the realm of high energy physics. Because of its importance to hadron spectroscopy, more precise data are needed.

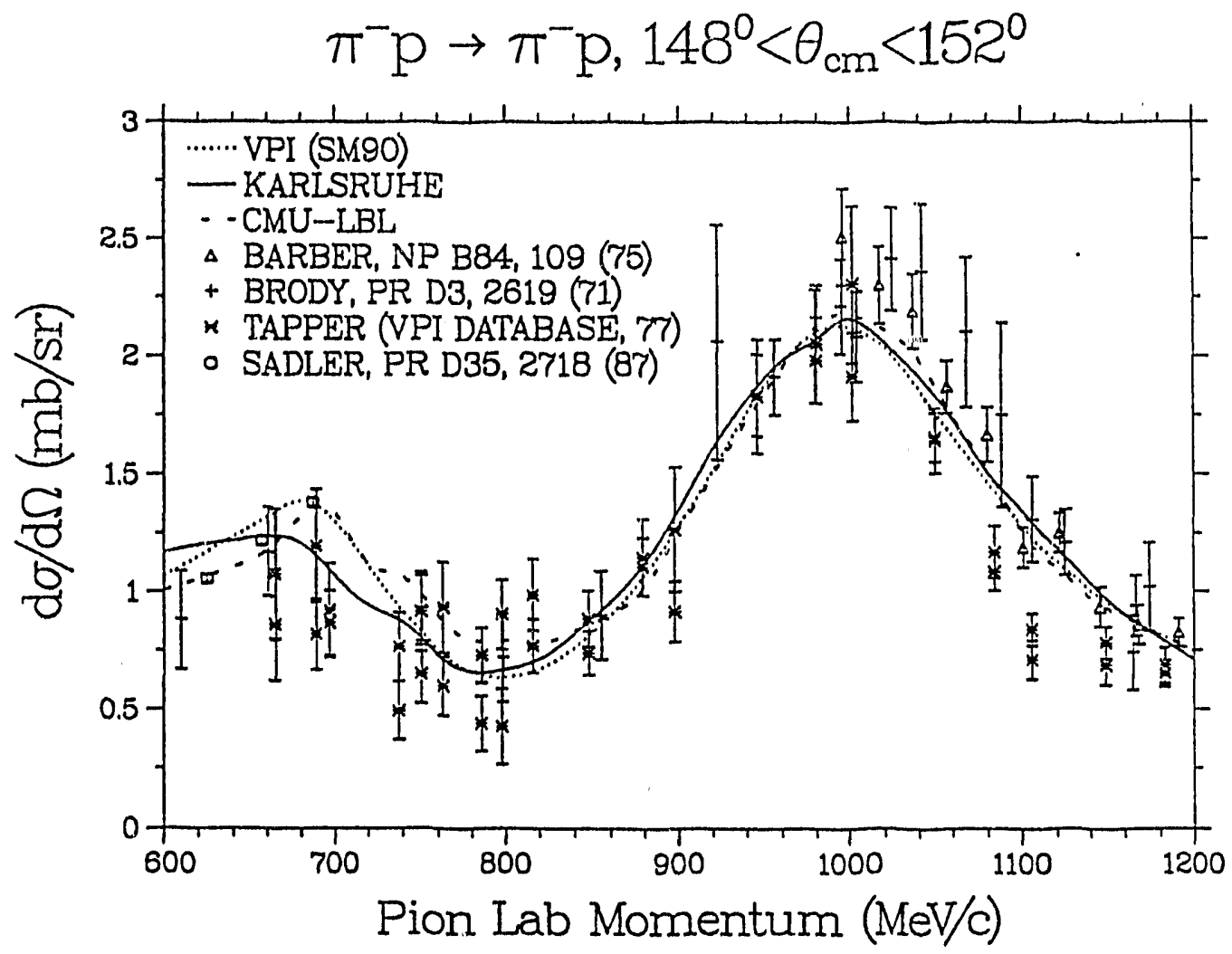

Figure 1 


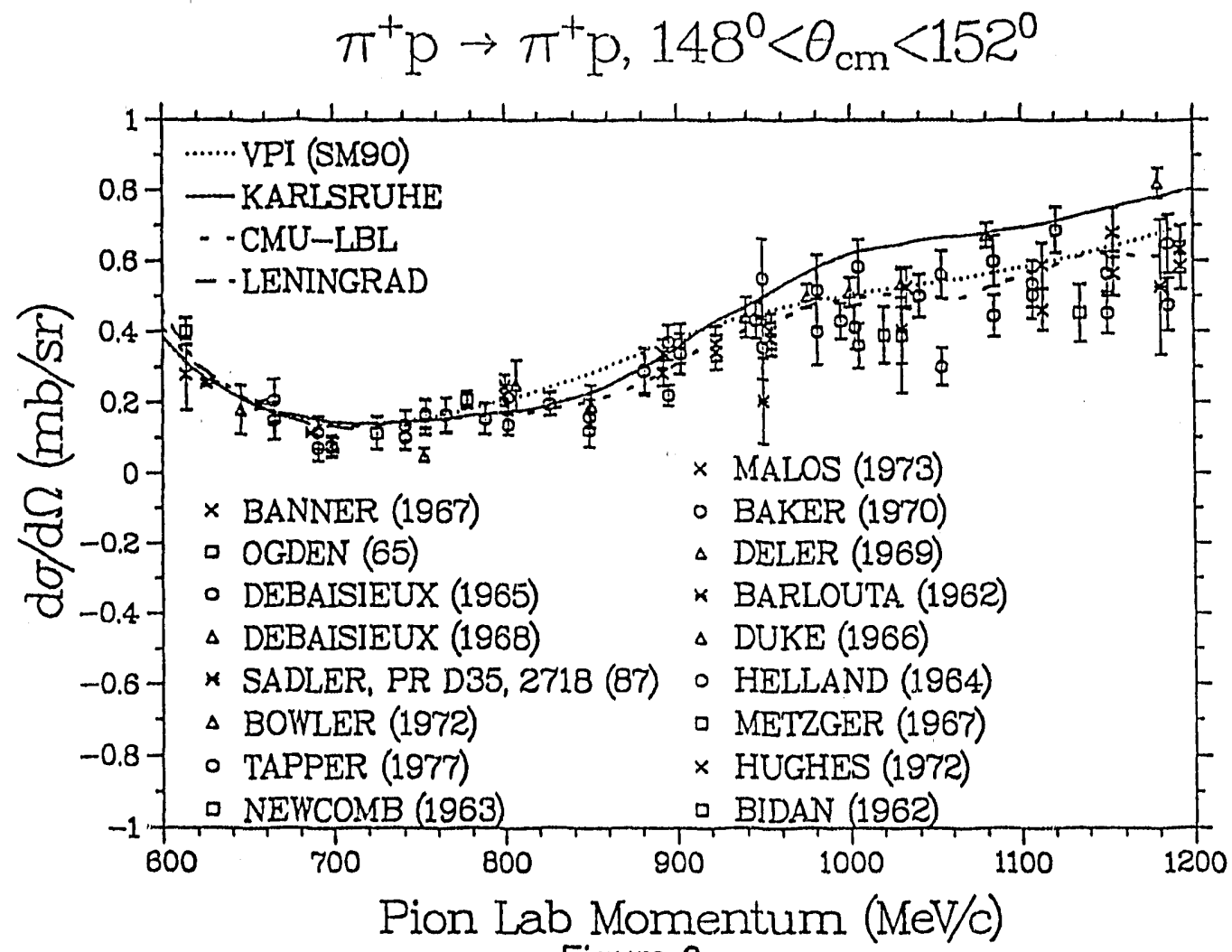

Figure 2

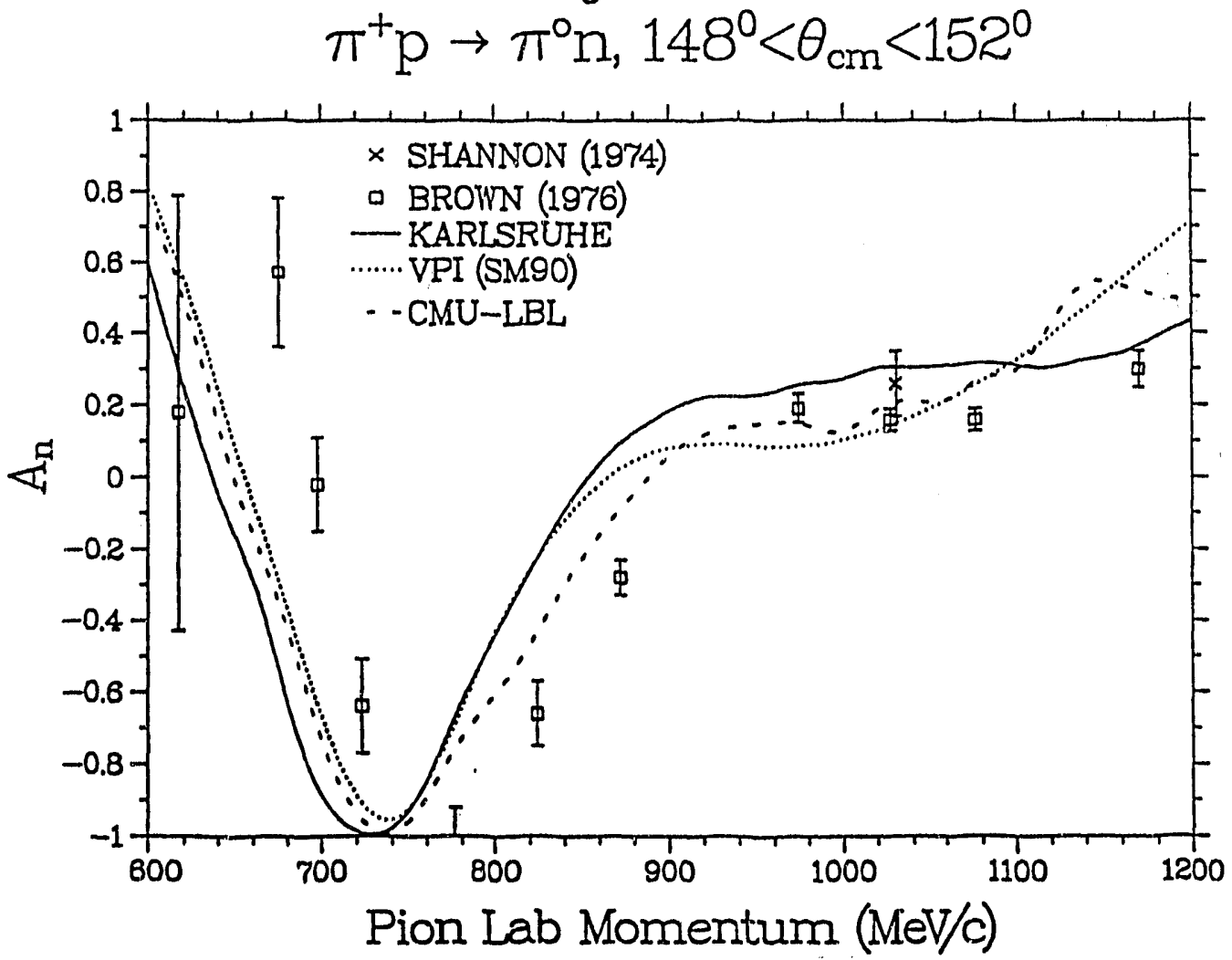

Figure 3 


\section{E. Design Work on the Superconducting Quadrupoles for PILAC}

Contributed by: Jeff E. Arrington, Ph.D.

In Collaboration with Arch Thiessen, MP-14, LAMPF

My research work for the summer was related to the design of the superconducting quads for the Pion Linear Accelerator (PILAC). Specifically, issues related to the identification of a better method of representing and computing the fringing fields of a large-bore superconducting quad were investigated. The research activity may be broken up into four tasks: work with RAYTRACE and the model it assumes, analysis of the magnetic field terms required to adequately represent the actual magnetic field, analysis of an alternative fringe-field function presented by Klaus Halbach, and early preparations for an experiment to measure the magnetic field from an existing magnet and extract magnetic field components from the resulting data.

The first task consisted of an introductory study of RAYTRACE. I spent a number of weeks studying the analytic work upon which RAYTRACE is based. The various components and their analytic models were studied, with the bulk of the time spent studying the quadrupole model and code. I worked on developing a mental picture of the magnets, understanding how the various input parameters were used in representing magnets, and in learning the flow of this program. A considerable part of the time was spent in attempting to use RAYTRACE to reproduce the work of Napolitano and Hunter1 (CEBAF) who used TOSCA. RAYTRACE in its native form generates magnetic field values only at the location of rays. I have written routines which drive RPOLES (a replacement for POLES which deletes the integration code), BPOLES, and BPLS. These routines also provide i/o for the RAYTRACE routines. With these auxiliary routines it is possible to generate magnetic field values at evenly spaced points in a cylindrical coordinate system to allow direct comparison with TOSCA values. A routine was also written to convert between cylindrical and rectangular coordinate systems in space and magnetic field. The results showed good agreement between the two codes for the $m=0,1$, and 2 components of the $B_{r}$ component of the quadrupole field. There is disagreement for the $m=3$ component of the field. My judgement is that the RAYTRACE generated $m=3$ component of the magnetic field does not show the relationship needed to represent a $z$ derivative of the $\mathrm{m}=2$ component. An additional component of this task was to attempt to find a new set of coefficients used to describe the Enge fitting function. The PILAC quads will be very similar to those at CEBAF, so we requested and received additional CEBAF data which we used to calculate new coefficients using Kaleidagraph's nonlinear least squares fitting routine. These coefficients were used in subsequent work with RAYTRACE. In a general sense, this task was successful because I do now have a 
better working knowledge of the theory, formalism and computational bases for RAYTRACE and superconducting quadrupole magnets. (Also, I have recently received additional data from Jim Napolitano which will allow me to proceed with the direct comparison of RAYTRACE/TOSCA results.)

The second task was an analysis of the terms in the analytically derived doublyinfinite sum1(Eq. 3) which actually contributes to the physical magnetic field. Data for the $n=6 m=0$ component, measured by Chris Morris, were scanned and digitized. These data were fit with four independent gaussian functions, and a functional representation of the data was obtained. From these data the $m=1$ and $m=2$ components were calculated and the ratios, e.g. $B_{r}(m=1, n=6) / B_{r}(m=0, n=6)$, of the magnetic field components corresponding to these terms were calculated. Plots of these ratios clearly showed that at least these terms must be included in the computer model. These ratios showed significant peaks in the region just downstream of the end of the iron pole pieces in each case, as well as at points further downstream. Another conclusion reached was that the data used could be better fit with 5 or 6 gaussians. Also, a rough Fourier transform performed by Mathematica indicated that up to $m=5$ might be required for the $n=6$ term to be adequately represented. Similar analysis for $n=4$ data showed that $m=1$ and 2 should also be considered in the computer model, since they make a contribution one order of magnitude smaller than the $\mathrm{m}=0$ component. The total $\mathrm{m}=4$ contribution is much smaller than the $\mathrm{n}=6$ component so that the effort involved in programming the terms into RAYTRACE may not be warranted. The actual recursion relation relates the function which represents the longitudinal variation of $m=0, b_{0}(z)$, to its second, fourth, sixth, etc. derivatives. 1 The first order approximation, which assumes that only $m=0$ and 1 are physically significant, results in the following differential equation:

$$
b_{o}(z)+C_{m n} r_{0}^{2} \frac{d^{2} b_{o}(z)}{d z^{2}}=G(z)
$$

where $G(z)$ is the $z$ variation observed in the $m=0$ data. Using a sum of gaussians for $G(z)$ produces a differential equation whose analytical solution is unusable. The numerical solution of this equation is also very difficult, since this is a stiff differential equation. I wrote three different basic routines for non-stiff equations, but none was able to solve the equation above. Stiffness means that if a part of the solution for $b_{0}(z)$ is $\exp (x)$ then numerical corruption by the $\exp (-x)$ solution (or vice-versa) is inevitable. The fact that the first order approximation was shown above to be insufficient makes it clear that solving this problem will not be trivial. This task was successful in showing that additions to current models are suggested by analysis of measured data. 
The third task arose due to comments made by Klaus Halbach at the workshop. He suggested that there are problems with Enge's fitting function having to do with its analyticity. Halbach proposed the function (refined after the meeting):

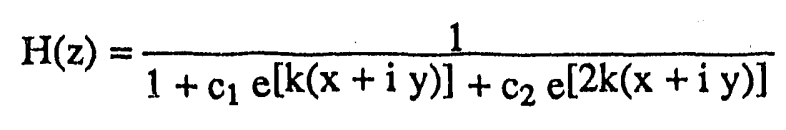

where $c_{1}$ and $c_{2}$ are fitting coefficients, and $k$ is $\pi /$ (half-gap). This function was fit to the Morris data to find $c_{1}, c_{2}$, and $k$, and then studied. These results were shown to the workshop on day two, and questions were raised by $\mathrm{H}$. Enge. At Enge's suggestion two sets of data were drawn from the paper2 he presented at the PILAC Optics workshop and used to compare the performance of the two functions. Both the Enge and the Halbach functions were found to have enough knobs to fit the data to the accuracy of the computer, giving the Halbach function an advantage in terms of simplicity. Both showed good behavior in fitting the pole edge. The Halbach function incorrectly showed the presence of a (mathematical) pole at 2 half-gaps in the $x$ plane, which precludes it from use as the general fitting function. Klaus has promised to continue to look for a fitting function.

We began to discuss plans to measure an existing magnet and perform an analysis similar to that performed by Chris Morris. Plans call for us to measure the total field at two points. I will be responsible for the analysis and programming needed to extract the $m, n$ components from the total field measurements.

Several people were involved in the work mentioned above. These included Arch Thiessen, Morris Klein, and Hal Butler of LANL and Klaus Halbach of Lawrence Berkeley Labs, all of whom have committed to be involved with this work on a continuing basis.

\section{References}

1. J. Napolitano and T. Hunter, "Calculations of Higher Multipole Components in a Large Superconducting Quadrupole Magnet," NIM.

2. H. A. Enge, "RAYTRACE and the Real World." 


\section{Report on leave of absence for L.D. Isenhower}

In late June of 1991, Dr. Isenhower returned from a leave of absence spent at the European Organization for Nuclear Research (CERN) located near Geneva, Switzerland on an NSF/NATO postdoctoral fellowship. During this appointment he worked on the Forward Ring Imaging Cherenkov (FRICH) detector1, which is one of the major subdetector systems on the DELPHI detector at the Large Electron Positron (LEP) collider. This device allows $\pi / K / p$ separation in the forward regions of the interaction point. This subdetector is undergoing tests of the final production version with one-half of one endcap recently installed for the 1992 collider run.

The DELPHI FRICH detector is an ambitious design and has required a number of innovations in its construction. It consists of 24 sections in each of the two detector endcaps. There is a liquid Cherenkov radiator in front of the drift chambers and a gas Cherenkov radiator behind the dritt chambers. For the gas radiator, mirrors are mounted to reflect and focus the UV light back onto the photosensitive gas in the drift chambers. The drift volume of each chamber has quartz widows on both sides to allow a single detector to be used for both liquid and gas radiators. The vacuum ultraviolet (VUV) Cherenkov photons are converted to photoelectrons by a tetrakis(dimethylamino)-ethylene (TMAE) gas mixture (the TMAE is kept at $35 \circ \mathrm{C}$ and the balance of the gas in the drift chambers is ethane), and these electrons are then drifted to a MWPC located in pockets situated radially around the detector. The drift length is up to $50 \mathrm{~cm}$ and the position of the VUV Cherenkov photon conversion is determined by timing the arrival of the electron to determine its distance from the wire chamber plane and by reading out active cathode pads to find the depth of conversion in the ethane-TMAE gas mixture.

The radius of the Cherenkov ring allows one to calculate the velocity of the particle that radiated the photon and since other DELPHI subdetectors measure the momentum, it is possible to identify the mass of the particle. The two main difficulties of RICH detectors of this design are that the long conversion length of the TMAE gas mixture requires a complicated "cloison" design2 to reduce optical feedback from secondary avalanches and that the electrons must drift in a crossed $E, B$ field, resulting in a large Lorentz angle (i.e., the electrons drift at a 50 degree angle from the direction of the drift field in the DELPHI FRICH). For the first problem it should be noted that the particle that emitted the Cherenkov light also passes through the chamber and produces about 600 electrons compared to the 15 or so electrons that are expected from the Cherenkov photons (i.e., one photoelectron for each photon). Since the detector must go in the endcap of a solenoidal magnet $(B=1.2$ Tesla), the latter problem is completely unavoidable. 
As part of the FRICH testing program, Isenhower worked on modeling its MWPC along with its drift box in order to study the effects of various problems encountered during the full-scale production of the detector. The most important part of this work was the use of TOSCA, a 3-D electromagnetic field calculation program utilizing finite element methods, to verify why severe focusing effects were being seen in the pit test conducted in 1990. These results were verified by measurements conducted at the University of Uppsala in Sweden and were used to guide the corrective work that was done to solve this problem. Isenhower will report on this work at the spring, 1992 meeting of the Texas Section of the APS. During this work, Isenhower also learned how to use the program GARFIELD to model wire chambers. These skills should be useful in any future involvement in detector construction.

Isenhower worked primarily with Olav Ullaland of CERN, who served as his scientific advisor. He also worked some with Henric Foeth, also of CERN. Working with these two scientists helped him to learn a large amount about small mistakes that can have an enormous impact on the performance of a detector. This knowledge will aid $A C U$ considerably in future detector work. Due to the small size of the program at $A C U$, it is not reasonable for it to actually build complex detector subsystems, but it is completely reasonable for it to test and model proposed detectors. The ability to model wire chamber performance will nicely compliment the ability to perform studies of detectors and their subcomponents with the tomography system described elsewhere in this report. By being able to do a good job of modeling wire/drift chambers, ACU should be able to do a better job of any tests done on these devices. This work will also be helpful when working with detectors in the future where drift chambers are involved.

References:

1. O. Botner et al., DELPHI Report 88-7 GEN 73 / RICH 30, 22 Feb. 1988.

2. W.Dulinski et al., Nucl. Inst. and Meth., A252 (1986) 418; R.Arnold et al., Nucl. Inst. and Meth. A252 (1986) 188; D. Bloch et al., Nucl. Inst. and Meth. A273 (1988) 847.

\section{Time Schedule}

A last concern to be addressed is how all of the above programs mesh together and how the investigators expect to be able to accomplish these tasks in their time available. Here we attempt to give a time line of what projects will finish when and where ACU expects to be at the end of this renewal period. Of course some of 
these projects are subject to delay and may not actually follow the plan given here, but It is necessary to show that it reasonable to undertake this set of programs. Some items do not appear in this time schedule since they will be ongoing concerns of the investigators during this grant. These include: (1) Sadler's continued work to incorporate $\pi \mathrm{N}$ complete data sets into partial wave analysis, (2) Development and refinement of tomography setup and software, (3) Plans for the next set of experiments with ACU involvement, (4) Yugoslav and Russian collaboration and visits, and (5) regular visits to LAMPF, FNAL, and CEBAF. It should also be noted that at this time the second half of FNAL E789 is very uncertain, since the fixed target program at FNAL could be delayed considerably or even killed.

Spring 1992: SPNPI data preliminary analysis completed.

Summer 1992: Papers for E882 submitted for publication. LAMPF NMS assembled (with at least $60 \mathrm{Csl}$ crystals and two conversion planes). Isenhower and Sadler each spend 4-6 weeks LAMPF helping on testing of the NMS, 3-4 weeks in Russia working on cusp experiment, and 1-2 weeks at CEBAF. Other summer work will be working on FNAL E789 RICH data analysis.

Fall/Winter 1992: Regular trips to LAMPF expected during this time for running of NMS experiments, possibly one trip each needed for work in Russia on cusp experiment.

Spring 1993: Continue work on preparation for additional LAMPF NMS experiments and possible work on upgrade of FNAL E789.

Summer 1993: Sadler and Isenhower will spend 4-6 weeks at CEBAF, with rest of summer likely working on NMS experiments at LAMPF. If FNAL E789 continues, Isenhower will take that work as his primary responsibility and Sadler will take the NMS work as his primary responsibility.

Fall/Winter 1993: Probable work on NMS experiments at LAMPF, with possible running of the second half of FNAL E789 beginning.

Spring 1994: Work should begin on more details of experiments to run at CEBAF. Planning should be beginning on PILAC experiments if this project obtains approval. 


\section{Student Contributions}

Undergraduate students have continued to play a signiflcant role in this research program. During the summer of 1991 two students, Scott Garner and Jason Phillips, spent two months in St. Petersburg working on the cusp experiment. Four students from ACU (John Keyser, Derek Lane, Randy Schnathorst and Gavin Williams) worked at Fermilab on E789 (Lane and Schnathorst were supported by LANL rather than ACU). Two students, Kelth Elmore and Tyson Browning, worked full time in Los Alamos with Jim Amann on the tomography setup. Two other students, Greg Loe and Andrew Rose, worked one month at LAMPF on the tomography set up and NMS development. Also three other students, Ernest Jones, James Redmon and Darien Tay, worked part-time for this contract during the summer at ACU.

Some work has been continued during the academic year. Randy Schnathorst has assisted in the software development for the RICH detector for Fermilab Exp. 789. James Redmon has aided in a number of projects, particularly with work on LAMPF E882 and E849. One student, Jason P'.illips, is doing an honors thesis on the Monte-Carlo modelling of the detection system used at SPNPI.

One tradition ACU is continuing is to have regular student contributions to local conferences. This spring six students will deliver papers at the Spring Meeting of the Texas APS to be held in San Marcos, Texas on March 6-7, 1992. Copies of their abstracts are attached following this section.

Four students from the last two graduating classes at ACU are pursuing graduate degrees in nuclear or particle physics. It is anticipated that this record will continue by some of the student authors whose abstracts are included below. 
Identification of the $n$-Mesen Production in the $\pi=0 \rightarrow \eta n$ Reaction at the Saint Petersburg Nuclear Physics Institute. Scott Garner, J. Phillips, M. Sadler, Abilene Christian University.-B. Nefkens, J. Price, D. White, University of California at Los Angeles.- S. Kruglov, I. Lopatin, V. Bekrenev, V. Abaev, A. Mayorov, Saint Petersburg Nuclear Physics Institute. - The $\eta$-meson production in the reaction $\pi-p \rightarrow \eta n$ was observed at the Saint Petersburg Nuclear Physics Institute in July 1991.

Measurements of this process were made by a collaboration of United States and Russian physicists to verify the SPNPI $\mathrm{P}$ (beam) and $\triangle \mathrm{P}$ (beam). Plastic scintillators were placed in front of and behind a liquid hydrogen target, a cylinder $10 \mathrm{~cm}$ in diameter. $A \pi^{-}$beam $3.5 \mathrm{~cm}$ wide by $1.5 \mathrm{~cm}$ high (FWHM) and $\triangle P / P=0.75 \%$ was passed through the target. A pion passing through the upstream counter only signified its interaction within the target. The upstream counter also served as the "start" for a neutron time of flight measurement. The "stop" was the detection of a neutron in one of the six neutron counters, placed $4.71 \mathrm{~m}$ from the target at $1,2,3,5,6$, and 7 degrees from the beam axis. TOF spectra were taken using a $P($ beam) ranging from 670 to 720 $\mathrm{MeV} / \mathrm{c}$ at these six angles. These TOF spectra showed peaks corresponding to $\pi^{\circ}$ and $\eta$ production.

Monte Carlo Simulation of the Acceptance for Neutrons From the $\pi \cdot n \rightarrow n n$ Reaction Near Threshold Jason Phillips, S. Garner, M. Sadler, Abilene Christian University.-B. Nefkens, J. Price, D. White, University of Callifornia at Los Angeles.- S. Kruglov, I. Lopatin, V. Bekrenev, V. Abaev, A. Mayorov, Saint Petersburg Nuclear Physics Institute. - The geometrical acceptance for neutrons from the $\pi \cdot p \rightarrow \eta n$ reaction has been modeled to analyze the results presented in the preceding talk. The Monte-Carlo program selects a beam particle from a phase space (spatial and momentum distribution) defined by the user. An interaction point within the $10-\mathrm{cm}-$ diameter cylindrical target and a random center-of-momentum scattering are selected. The acceptance for these neutrons by six forward-angle $\left(1^{\circ}-7^{\circ}\right)$ neutron counters is determined by the ratio of the number of hits to total events. These calculations were done at $687 \mathrm{MeV} / \mathrm{c}$ (threshold) to $720 \mathrm{MeV} / \mathrm{c}$. The results were then weighted by the cross section which was assumed to be a linear function of center of mass momentum and then compared to the actual measurements taken at SPNPI. Use of this technique to determine the centroid and width of the beam momentum distribution will be discussed. Plans for future simulations in this program, including the acceptance for gamma rays from either $\pi^{\circ}$ or $\eta$ decay, will be discussed. 
Monte Carle Modeling of the Absolute Solid Angle Acceptance in the LAMPF Plo Spectrometer J.A. Redmon, L.D. Isenhower, M.E. Sadler, Abilene Christian University _ Meade Brooks, Texas Tech University_..- The PIO Spectrometer is used to measure the individual energles of the two gamma rays produced by $\pi^{\circ}$ decay. With these energies and the opening angle between the two gamma rays, the energy of the $\pi^{\circ}$ can be calculated. An absolute calculation of the solid angle is needed for differential cross section calculations. Using Monte Carlo modeling, the laboratory setup is scrutinized by changing various parameters in the simulation runs. This model is compared with actual data obtained by experiment. Using this comparison, it is possible to determine any deviations in the laboratory setup. With the deviations known, an absolute solid angle acceptance can be computed. Parameters varied are the following: 1) Beam Energy, 2) Beam Position in the $X$ and $Y$ direction relative to the target, and 3 ) Target Position in the $Z$ direction relative to the pivot between the two arms of the spectrometer. These variations are made for beam energies of 10,20 , and $40 \mathrm{MeV}$ at laboratory scattering angles of 0 and 180 degrees.

Measuring Velecity with the "Optic Boom": The Contribution of the Bing Imaging Cerenkov Detector to the Search for Charmless Beauty Decay at Fermilab. Randy Schnathorst and the E789 collaboration (Abilene Christian U., FNAL, Institute of Physics-Taiwan, LANL, Lawrence Berkelex, Northern llinois $U, U$. of , hicago, $U$. of South Carolina). - A charged particle traveling in a medium with a speed exceeding the speed of light in that medium produces Cerenkov radiation (light). This effect is, in a very loose sense, the optical analogue of a sonic boom. The light is emitted at a specific angle, $\theta$, determined by the velocity, $\beta$, of the particle according to the equation $\beta=1 /(n \cos \theta)$ where $n$ is the index of refraction of the medium. In the Ring Imaging Cerenkov Detector (RICH), light emitted at an angle $\theta$ is focused as a ring of radius $r=f \tan \theta$ ( $f=$ focal length) onto a detector. The velocity of the particle is calculated from the radius of the circular ring of light striking the detector, allowing the mass of the particle to be determined. E789 makes use of the well-known E605 RICH detector to study D-and B-meson decays. The physics explored by E789 and the operation of the RICH will be described, and images produced by analysis of data from the $\mathrm{RICH}$ will be presented. 


\section{Tomography Apparatus for the Evaluation of the Three-Dimensional}

Response of Scintillation Detectors Using Cosmic Muons, Keith Elmore, Tyson R. Browning, M.E. Sadler, Abilene Christian University - Jim Amann, Mark Whitton, Los Alamos National Laboratony. - Many physics experiments use scintillation detectors such as $\mathrm{Nal}, \mathrm{CsI}$ and BGO as calorimeters to measure the energy of particles. The energy resolution has been limited either by the ability of the crystal to contain the produced electromiagnetic showers or by the limits set by the number of photoelectrons produced in the photomultiplier. Concern has recently arisen regarding the uniformity of response for any given scintillator. The question of uniformity becomes extremely important when the scintillators will be used in large detector arrays, such as spectrometers. Tomography techniques which allow a 3dimensional analysis of crystals will be discussed, both past efforts ${ }^{1}$ and improvements. The new ideas have been implemented in an apparatus currently being used to analyze the 3-D response of Csl crystals (to be incorporated into the Neutral Meson Spectrometer being built at LAMPF). This talk will discuss these concepts and will be followed by a presentation of the apparatus and techniques needed to make these analyses.

1 D. H. Dowell, et al., Nucl. Inst. and Meth. A286, 183 (1990).

Data Acquisition and Analysis for a Tomegraphy Apparatus for the Evaluation of the Three-Dimensional Response of Scintillation Detectors Using Cosmic Muons. Tyson R. Browning, Keith Elmore, M.E. Sadler, Abilene Christian University.--Jim Amann, Mark Whitton, Los Alamos National Laboratony.--An apparatus has been built and is currently being used to analyze the 3-D response of Csl crystals which will be incorporated into the Neutral Meson Spectrometer being built at LAMPF. With the setup, the response from volumes as small as one cubic centimeter can readily be analyzed. This talk will continue the ideas presented in the previous talk, discussing the apparatus and electronics needed to make these analyses. Furthermore, the data acquisition devices and software used will be explained. Methods of analyzing the data using Fourier transform techniques will be discussed. This project is still underway: results obtained thus far will be shown; status and future directions will be presented; applications for future use will be discussed and solicited. 


\section{Publications:}

Publications in refereed journals, contributions to topical conferences, seminar presentations and papers presented at professional meetings since 1985 are listed below in reverse chronological order. Included in this list are publications with Isenhower as a co-author which resulted from his work during his leave of absence at CERN, as well as work done as a graduate student at lowa State University/Ames Laboratory. Even though this work was not supported by this grant, it is included here since it involves one of the principal investigators.

Articles In Refereed Journals:

The Spin Rotation Parameters $A$ and $R$ from $\pi+p$ and $\pi \cdot p$ Elastic Scattering from 427 to $657 \mathrm{MeV} / \mathrm{c}$, I. Supek, D.B. Barlow, W.J. Briscoe, J.F. Davis, G.J. Kim, D.W. Lane, A. Mokhtari, B.M.K. Nefkens, C. Pillai, M.E. Sadler, C.J. Seftor, M.F. Taragin, J.A. Wightman, submitted to Phys. Rev. D (1992).

On the Extraction of the $\pi N N$ Coupling Constant from Partial Wave Analysis, J. Stahov, M. E. Sadler and V.V. Abaev, Phys. Rev. Lett., Vol. 63, No. 4, 548 (1992).

A Test of Consistency of Low-Energy Pion-Nucleon Differential Cross Sections with Partial Cross Sections, T. S. Hill, Journal of Undergraduate Research in Physics, Vol. 9, No. 2, 53 (May, 1991).

Angle and Energy Dependence of the Superratio for $\pi^{+}$and $\pi$ - Elastic Scattering from ${ }^{3} \mathrm{H}$ and $3 \mathrm{He}$ : Evidence for Charge-Symmetry Violation, C. Pillai, D. B. Barlow, B. L. Berman, W. J. Briscoe, A. Mokhtari, B. M. K. Nefkens and M. E. Sadler, Phys. Rev. C43, 1838 (1991).

Measurement of the Left-Right Asymmetry in $\pi p \rightarrow$ m from 301-625 MeV/C at Backward Angles, G. J. Kim, J. Arends, W. J. Briscoe, J. Engelage, B. M. K. Nefkens, M. E. Sadler and H. J. Ziock, Phys. Rev. D43, 687 (1991).

The Reaction Pomeron-Pomeron $\rightarrow \pi+\pi$ and an Unusual Production Mechanism for the $f_{2}(1270)$, By Ames-Bologna-CERN-Dortmund-Heidelberg-Warsaw Collaboration (A. Breakstone, et al.), Z. Phys. C48, 569 (1990).

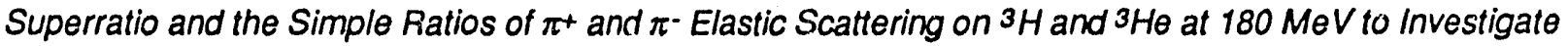
Charge Symmetry, B. M. K. Nefkens, W. J. Briscoe, A. D. Eichon, D. H. Fitzgerald, A. Mokhtari, J. A. Wightman and M. E. Sadler, Phys. Rev. C41, 2770 (1990).

Analyzing Power for $\pi p$ Charge Exchange in the Backward Hemisphere from 301 to $625 \mathrm{MeV} / \mathrm{C}$ and a Test of $\pi N$ Partial-Wave Analyses, G. J. Kim J. Arends, W. I Briscoe, J. Engelage, B. M. K. Nefkens, M. E. Sadler, M. Taragin and H. J. Ziock, Physical Review D41, 733 (1990).

A measurement of the lifetime of the tau lepton, By DELPHI Collaboration (P. Abreu, W. Adam, F. Adami, T. Adye, T. Akesson, G.D. Alekseev, P. Allen, S. Almehed, S.J. Alvsvaag, U. Amaldi, E. Anassontzis, P. Antilogus, W.D. Apel, R.J. Apsimon, B. Asman, P. Ast:er, J.E. Augustin, A. Augustinus, P. Baillon, P. Bambade, F. Barao, G. Barbiellini, D.Yu. Bardin, A. Baroncelli, O. Barring, W. Bartl, M. Battaglia, M.J. Bates, M. Baubillier, K.H. Becks, C.J. Beeston, M. Begalli, P. Beilliere, Yu. Belokopytov, P. Beltran, D. Benedic, J.M. Benlloch, M. Berggren, D. Bertrand, F. Bianchi, J.H. Bibby, M.S.Bilenky, P. Billoir, J. Bjarne, D. Bloch, S. Blyth, P. Bogolyubov, T. Bolognese, M. Bonapart, M. Bonesini, W. Bonivento, P.S.L. Booth, M. 
Boratav, P. Borgeaud, G. Borisov, H. Borner, C. Bosio, B. Bostjancic, O. Botner, B. Bouquet, M. Bozzo, S. Braibant, P. Branchini, K.D. Brand, R.A. Brenner, C. Bricman, R.C.A. Brown, N. Brummer, J.M.Brunet, L. Bugge, T. Buran, H. Burmeister, J.A.M.A. Buytaert, M.Caccia, M. Calvi, A.J. Camacho Rozas, J.E. Campagne, A. Campion, T. Camporesi, V. Canale, F. Cao, F. Carena, L. Carroll, C. Caso, E. Castelli, M.V. Castillo Gimenez, A. Cattal, F.R. Cavallo, L. Cerrito, A. Chan, P. Charpentier, P. Checchia, G.A. Chelkov, L. Chevalier, P. Chliapnikov, V. Chorowicz, R. Cirio, M.P. Clara, P. Collins, J.L. Contreras, R. Contri, G.

Cosme, F. Couchot, H.B. Crawley, D. Crennell, G. Crosettl, N. Crosland, M. Crozon, J. Cuevas Maestro, S. Czellar, S. Dagoret, E. Dahl-Jensen, B. D'Almagne, M. Dam, G. Damgaard, G. Darbo, E. Daubie, P.D. Dauncey, M. Davenport, P. David, A. De Angelis, M. De Beer, H. De Boeck, W. De Boer, C. De Clercq, M.D.M. De Fez Laso, N. De Groot, C. de la Vaissiere, B. De Lotto, A. De Min, C. Defoix, D. Delikaris, S. Delorme, P. Delpierre, N. Demaria, J. Derkaoui, L. Di Ciaccio, H. Dijkstra, F. Djama, J. Dolbeau, M. Donszelmann, K. Doroba, M. Dracos, J. Drees, M. Dris, Y. Dufour, W. Dulinski, L.O. Eek, P.A. Eerola, T. Ekelof, G. Ekspong, A. Elliot Peisert, J.P. Engel, V. Falaleev, D. Fassouliotis, M. Fernandez Alonso, A. Ferrer, T.A. Filippas, A. Firestone, H. Foeth, E. Fokitis, P. Folegati, F. Fontanell, K.A.J. Forbes, H. Forsbach, B. Franek, P. Frenkiel, D.C. Fries, A.G. Frodesen, R. Fruhwirth, F. Fulda-Quenzer, K. Furnival, H. Furstenau, J. Fuster, J.M. Gago, G. Galeazzi, D. Gamba, C. Garcia, J. Garcia, U. Gasparini, P. Gavillet, E.N. Gazis, J.P. Gerber, P. Giacomelli, K.W. Glitza, R. Gokieli, V.M. Golovatyuk, J.J. Gomez y Cadenas, A. Goobar, G. Gopal, M. Gorski, V. Gracco, A. Grant, F. Grard, E. Graziani, M.H. Gros, G. Grosdidier, E. Gross, B. Grossetete, S. Gumenyuk, J. Guy, F. Hahn, M. Hahn, S. Haider, Z. Hajduk, A. Hakansson, A. Hallgren, K. Hamacher, G. Hamel de Monchenault, F.J. Harris, B.W. Heck, T. Henkes, I. Herbst, J.J. Hernandez, P. Herquet, H. Herr, I. Hietanen, C.O. Higgins, E. Higon, H.J. Hilke, S.D. Hodgson, T. Hofmokl, R. Holmes, S.O. Holmgren, D. Holthuizen, P.F. Honore, J.E. Hooper, R. Horisberger, M. Houlden, J. Hrubec, P.O. Hulth, K. Hultqvist, D. Husson, B.D. Hyams, P. loannou, D. Isenhower, P.S. Iversen, J.N. Jackson, P. Jalocha, G. Jarlskog, P. Jarry, B. Jean-Marie, E.K. Johansson, D. Johnsson, M. Jonker, L. Jonsson, P. Juillot, G. Kalkanis, G. Kalmus, G. Kantardjian, F. Kapusta, S. Katsanevas, E.C. Katsoufis, R. Keranen, J. Kesteman, B.A. Khomenko, N.N. Khovanskii, B. King, N.J. Kjaent, H. Klein, W. Klempt, A. Klovning, P. Kluit, J.H. Kohne, B. Koene, P. Kokkinias, M. Kopf, M. Koratzinos, K. Korcyl, A.V. Korytov, B. Korzen, V. Kostukhin, C. Kourkoumelis, T. Kreuzberger, J. Krolikowski, U. Kruner-Marquis, W. Krupinski, W. Kucewicz, K. Kurvinen, C. Lacasta, C. Lambropoulos, J.W. Lamsa, L. Lanceri, V. Lapin, J.P. Laugier, R. Lauhakangas, G. Leder, F. Ledroit, J. Lemonne, G. Lenzen, V. Lepeltier, A. Letessier-Selvon, D. Liko, E. Lieb, E. Lillethun, J. Lindgren, A. Lipniacka, I. Lippi, R. Llosa, B. Lorstad, M. Lokajicek, J. Loken, M.A. Lopez Aguera, A. Lopez-Fernandez, M. Los, D. Loukas, A. Lounis, J.J. Lozano, R. Lucock, P. Lutz, L. Lyons, G. Mahlum, J. Maillard, A. Maltezos, S. Maltezos, F. Mandl, J. Marco, M. Margoni, J.C. Marin, A. Markou, S. Marti, L. Mathis, F. Matorras, C. Matteuzzi, G. Matthiae, M. Matveev, M. Mazzucato, M. McCubbin, R. McNuity, E. Menichetti, G. Meola, C. Meroni, W.T. Meyer, M. Michelotto, W.A. Mitaroff, G.V. Micelmacher, U. Mjornmark, T. Moa, R. Moller, K. Monig, M.R. Monge, P. Morettini, H. Muller, H. Muller, W.J. Murray, G. Myatt, F. Naraghi, U. Nau-Korzen, F.L. Navarria, P. Negri, B.S. Nielsen, B. Nijighar, V. Nikolaenko, V. Obraztsov, A.G. Olshevski, R. Orava, A. Ostankov, A. Ouraou, R. Pain, H. Palka, T. Papadopoulou, L. Pape, A. Passeri, M. Pegoraro, V. Perevozchikov, M. Pernicka, A. Perrotta, F. Pierre, M. Pimenta, O. Pingot, A. Pinsent, M.E. Pol, G. Polok, P. Poropat, P. Privitera, A. Pullia, J. Pyyhtia, D. Radojicic, S. Ragazzi, P.N. Ratotf, A.L. Read, N.G. Redaelli, M. Regler, D. Reid, P.B. Renton, L.K. Resvanis, F. Richard, M. Richardson, J. Ridky, G. Rinaudo, I. Roditi, A. Romero, I. Roncagliolo, P. Ronchese, C. Ronnquist, E.I. Rosenberg, U. Rossi, E. Rosso, P. Roudeau, T. Rovelli, W. Ruckstuhl, V. Ruhlmann, A. Ruiz, K. Rybicki, H. Saarikko, Y. Sacquin, J. Salt, E. Sanchez, J. Sanchez, M. Sannino, M. Schaffer, S. Schael, H. Schneider, M.A.E. Schyns, F. Scuri, A.M. Segar, R. Sekulin, M. Sessa, G. Sette, R. Seufert, R.C. Shellard, P. Siegrist, S. Simonetti, F. Simonetto, A.N. Sisakian, T.B. Skaali, G. Skjevling, G. Smadja, G.R. Smith, N. Smimov, R. Sosnowski, T.S. Spassof, E. Spiriti, S. Squarcia, H. Stack, C. Stanescil, G. Stavropoulos, F. Stichelbaut, A. Stocchi, J. Strauss, R. Strub, C.J. Stubenrauch, M. Szczekowski, M. Szeptycka, P. Szymanski, T. Tabarelli, S. Tavernier, G. Theodosiou, A. Tilquin, J. Timmermans, V.G. Timofeev, L.G. Tkachev, T. Todorov, D.Z. Toet, L. Tortora, M.T. Trainor, D. Treille, U. Trevisan, W. Trischuk, G. Tristram, C. Troncon, A. Tsirou, E.N. Tsyganov, M. Turala, R. Turchetta, M. 
Turluer, T. Tuuva, I.A. Tyapkin, M. Tyndel, S. Tzamarias, B. Uberschar, S. Uberschar, O. Ullaland, V.A. Uvarov, G. Valenti, E. Vallazza, J.A. Valls Ferrer, G.W. van Apeldoorn, P. van Dam, W.K. Van Doninck, N. van Eijndhoven, C. Vander Velde, J. Varela, P. Vaz, G. Vegni, L. Ventura, W. Venus, F. Verbeure, L.S. Vertogradov, L. Vibert, D. Vilanova, E.V. Vlasov, A.S. Vodopyanov, M. Vollmer, S. Volponi, G. Voulgaris, M. Voutilainen, V. Vrba, H. Wahlen, C. Walck, F. Waldner, M. Wayne, P. Weilhammer, J. Werner, A.M. Wetherell, J.H. Wickens, J. Wikne, G.R. Wilkinson, W.S.C. Williams, M. Winter, D. Wormald, G. Wormser, K. Woschnagg, N. Yamdagni, P. Yepes, A. Zaitsev, A. Zalewska, P. Zalewski, D. Zavrtanik, E. Zevgolatakos, G. Zhang, N.I. Zimin, M. Zito, R. Zitoun, R. Zukanovich Funchal, G. Zumerle, J. Zuniga (Ames Lab \& lowa State U. \& Antwerp U. \& Brusseis U., IIHE \& Mons U. \& Athens U., Nucl. Phys. Lab \& Bergen U. \& Bohr Inst. \& Bologna U. \& INFN, Bologna \& College de France \& CERN \& Strasbourg, CRN \& Democritos Nuclear Research Center \& Genoa U. \& INFN, Genoa \& Helsinki U. \& Dubna, JINR \& Karlsruhe U., IEKP \& Cracow, INP-Exp \& Orsay, LAL \& Lisbon, IFM \& Liverpool U. \& Paris, Curie Univ. VI, LPNHE \& Paris, Univ. VII, LPNHE \& Lund U. \& Lyon, IPN \& Madrid U. \& Marseille, CPPM \& Milan U. \& INFN, Milan \& Bohr Inst. \& NIKHEF, Amsterdam \& Athens, Tech. U. \& Oslo U. \& Oxford U., NPL \& Padua U. \& INFN, Padua \& Rutherford \& Rome U., TorVergata \& INFN, Rome \& Saclay \& Rome, ISS \& Cantabria U., Santander \& Serpukhov, IFVE \& Stockholm U. \& Turin U. \& INFN, Turin \& Trieste U., Ist. Fis. \& INFN, Trieste \& Udine U. \& Uppsala U. \& Valencia U. \& Vienna, OAW \& Warsaw U., IEP \& Warsaw, Inst. Nucl. Studies \& Wuppertal U.), Phys. Lett. B267, 422 (1991).

A study of the reaction $e^{+} \theta^{-} \rightarrow \mu^{+} \mu^{-}$around the Zo pole, By the DELPHI Collaboration (P. Abreu, et al.), Phys. Lett., B260, 240 (1991).

Production of strange particles in the hadronic decays of the ZO, By the DELPHI Collaboration (P. Abreu, et al.), CERN-PPE-91-207, Nov 1991, 19pp. (Submitted to Phys. Lett. B).

A measurement of the $\mathrm{b} \overline{\mathrm{b}}$ forward backward asymmetry using the semileptonic decay into muons, By the DELPHI Collaboration (P. Abreu, et al.), CERN-PPE-91-213, Dec 1991, 16pp. (Submitted to Phys. Lett. B).

A measurement of $\sin ^{2} \theta_{w}$ from the charge asymmetry of hadronic events at the Zo peak, By DELPHI Collaboration (P. Abreu, et al.), CERN-PPE-91-211, Nov 1991, 18pp. (Submitted to Phys. Lett. B).

Multiplicity dependence of mean transverse momentum in $\theta^{+} \theta^{-}$annihilations at LEP energies, By DELPHI Collaboration (P. Abreu, et al.), CERN-PPE-91-194, Nov 1991, 16pp. (Submitted to Phys. Lett. B).

Searches for heavy neutrinos from $Z$ decays, By DELPHI Collaboration (P. Abreu, et al.), CERN-PPE-91175, Oct 1991, 16pp. (Submitted to Phys. Lett. B).

Determination of $\alpha_{s}$ in second order QCD in hadronic $Z$ decays, By DELPHI Collaboration (P. Abreu, et al.), CERN-PPE-91-181, Oct 1991, 45pp.

(Submitteci to Z. Phys. C).

Study of final state photons in hadronic zo decay and limits on new phenomena, By DELPHI Collaboration (P. Abreu, et al.), CERN-PPE-91-174, Oct 1991, 24pp. (Submitted to Z. Phys. C).

Measurement of the average lifetime of $B$ hadrons, By DELPHI Collaboration (P. Abreu, et al.), CERN-PPE91-131, Aug 1991, 32pp. (Submitted to Z. Phys. C).

A search for neutral higgs particles in 20 decays, By DELPHI Collaboration (P. Abreu, et al.), CERN-PPE91-132, Aug 1991, 32pp (Submitted to Nucl. Phys. B). 
Search for scalar leptoquarks from Z0 decays, By DELPHI Collaboration (P. Abreu, et al.), CERN-PPE-91138, Aug 1991, 13pp. (Submitted to Phys. Lett. B).

The reaction $\theta+\theta-\rightarrow \gamma \gamma$ at $Z 0$ energies, By DELPHI Collaboration (P. Abreu, et al.), CERN-PPE-91-109, Jul 1991, 16pp. (Submitted to Phys. Lett. B).

Study of orientation of 3 jet events in zO hadronic decays using the delphi detector, By DELPHI Collaboration (P. Abreu, et al.), CERN-PPE-91-79, May 1991,15pp. (Submitted to Phys. Lett. B).

Charged Particle Multiplicity Distributions in Restricted Rapidity Intervals in Zo Hadronic Decays, By the DELPHI Collaboration (P. Abreu, et al.), CERN PPE 91-78, Subm. to Z. Phys. C, Dedicated to the memory of L Van Hove.

Search for Excited Charged Leptons in Z० Decays, By the DELPHI Collaboration (P. Abreu, et al.), CERN PPE 91-100, Subm. to Z. Phys. C.

Determination of Z० Resonance Parameters and Couplings from its Hadronic and Leptonic Decays, By the DELPHI Collaboration (P. Abreu, et al.), Nucl. Phys. B367, 511 (1991).

Higher Order QCD Effects and Particle Density in Full Phase Space from High $p_{t}$ Interactions at the ISR, By Ames-Bologna-CERN-Dortmund- Heidelberg-Warsaw Collaboration (A. Breakstone, R. Campanini, G. Claesson, H.B. Crawley, M. Cuffiani, G.M. Dallavalle, M.M. Deninno, K. Doroba, D. Drijard, F. Fabbri, A. Firestone, M.A. Faessler, H.G. Fischer, H. Floge, S.Y. Fung, H. Frehse, S. Garpman, W. Geist, L. Gesswein, G. Giacomelli, R. Gokieli, M. Gorbics, M. Gorski, C.R. Gruhn, P. Hanke, M. Heiden, W. Herr, D. Isenhower, T.J. Ketel, E.E. Kluge, J.W. Lamsa, T. Lohse, I. Lund, R. Mankel, W.T. Meyer, T. Nakada, I. Otterlund, M. Panter, H. Pugh, A. Putzer, K. Rauschnabel, B. Rensch, F. Rimondi, M. Schmelling, L. Schroder, G.P. Siroli, R. Sosnowski, E. Stenlund, T.J.M. Symons, M. Szczekowski, R. Szwed, O Ullaland, , R. Walczak, D. Wegener, M. Wunsch (Ames Lab \& LBL, Berkeley \& Bologna U. \& INFN, Bologna \& CERN \& Dortmund U. \& Heidelberg U., IHEP \& Lund U. \& Warsaw U., IEP \& Warsaw, Inst. Nucl. Studies), CERN PPE 91-59, Subm. to Z. Phys. C.

Exclusive Neutral Strange Particle Production in Double Pomeron Exchange Reactions from Proton-Proton Interactions at $\sqrt{s}=62 \mathrm{GeV}$, By Ames-Bologna-CERN-Dortmund-Heidelberg-Warsaw Collaboration (A. Breakstone, et al.), CERN PRE 90-008, Subm. to Z. Phys. C

The Reaction Pomeron-Pomeron $\rightarrow \pi+\pi$ and an Unusual Production Mechanism for the $f_{.2}(1270)$, By Ames-Bologna-CERN-Dortmund-Heidelberg-Warsaw Collaboration (A. Breakstone, et al.), Z. Phys. C48, 569 (1990).

A Sensitive Test of QCD from Parton-Parton Scattering at the ISR CERN, By Ames-Bologna-CERN-Dortmund-Heidelberg-Warsaw Collaboration (A. Breakstone, et al.), Phys. Lett., 248B, 220 (1990).

A Sensitive Test of QCD from Parton-Parton Scattering at the ISR, By Ames-BolognaCERN-Dortmund-Heidelberg-Warsaw Collaboration (A. Breakstone, et al.), CERN-EP/90-74, June 1990 (submitted to Phys. Lett. B).

Exclusive Neutral Strange Particle Production in Double Pomeron Exchange Reactions from ProtonProton Interactions at $\sqrt{ } s=62 \mathrm{GeV}$, By Ames-Bologna-CERN- Dortmund-Heidelberg-Warsaw Collaboration (A. Breakstone, et al.), IS-J-3745, Fab. 1990 (submitted to Z. Phys. C). 


\section{Contrlbutlons to Toplcal Conferences:}

Fonward-and Backward-angle Differential Cross Sections For $\pi \mathrm{p} \rightarrow \pi^{\circ} \mathrm{n}$ at $T_{\pi}=10,20$, and $40 \mathrm{MeV}$, M.E. Sadler, B.M. Brooks, L.D. Isenhower, W.J. Briscoe, J.D. Bowman, D.H. Fitzgerald, J.N. Knudson, Presented at the IV Initernational Symposium on Pion-Nucleon Physics and the Structure of the Nucleon, Bad Honititi, Germany, Sept. 9-13, 1991.

Equality Test of Istsspin Invariance Using Complete Data Sets for Pion-Nucleon Scattering, M.E. Sadler, Presented at the IV International Symposium on Pion-Nucleon Physics and the Structure of the Nucleon, Bad Honnef, Germany, Sept. 9-13, 1991.

Baryon Resonance Studies at the Proposed PILAC Facility, M.E. Sardier, Presented at the Baryon Spectroscopy and the Structure of the Nucleon Conference held at Laboratoire National Saturne, France, Sept. 23-25, 1991.

Operation of silicon microstrip detectors in a high radiation environment, By J.S. Kapustinsky, D.M. Alde, J.G. Boissevain, R.G. Jeppesen, D.W. Lane, M.J. Leitch, J.W. Lillberg, T.A. Lopez, P.L. McGaughey, J.M. Moss, J.C. Peng (Los Alamos), B.M. Brooks, L.D. Isenhower, M.E. Sadler (Abilene Christian U.), L.M. Lederman, M.H. Schub (Chicago U.), C.N. Brown, W.E. Cooper, K. Gounder, Y.B. Hsiung, C.S. Mishra (Fermilab), G. Gidal, M.S. Kowitt, K.B. Luk (LBL, Berkeley), D.M. Kaplan, V.M. Martin, R.S. Preston, J. Sa (Northern Illinois U.), R.L. Childers, C.W. Darden (South Carolina U.), G.C. Kiang, P.K. Teng (Taiwan, Inst. Phys.), FERMILAB-CONF-90-214-E, Oct 1990. 4pp. Presented at Symp. on Detector Research and Development for the Super Collider, Ft. Worth, TX, Oct 15-18, 1990.

Dilepton and dihadron production in proton - nucleus collisions at 800-gev, By E-772 Collaboration and E789 Collaboration (C.S. Mishra, et al.), FERMILAB-CONF-90/100-E, May 1990. 12pp. Presented at the XXVth Rencontres de Moriond, High Energy Hadronic Interactions, Les Arcs, France, Mar 11-17, 1990.

Performance of a Silicon Microstrip Detector in a High Radiation Environment, C. S. Mishra, C. N. Brown, J. Kapustinsky, M. J. Leitch, P. L. McCaughey, J. C. Peng, W. Sailor, K. Holzscheiler and M. Sadler, 15th APS Division of Particles and Fields General Meeting, Houston, 3-6 January, 1990.

\section{Seminars:}

Baryon Resonances at PILAC, M. E. Sadler at the Institute for Nuclear Theory, University of Washington, Seattle, 6 January 1992.

The World's First Complete Measurements in the Pion-Nucleon System, M. E. Sadler, University of Warsaw, Poland, 31 May 1991. 
Papers at professional meetings (presented by first author):

Back-Angle Cross Sections for $\pi$ Scattering from ${ }^{3} \mathrm{H}$ and ${ }^{3} \mathrm{He}$ near the $\Delta$ Resonance, S. J. Greene, W. J. Briscoe, B. L. Berman, R. W. Caress, K. S. Dhuga, S. N. Dragic, S. K. Matthews, N. J. Nicholas, D. B. Barlow, B. M. K. Nefkens, C. Pillai, J. Price and M. E. Sadler, submitted to Int. Nucl. Phys. Conf., Wiesbaden, Germany, 26 July-1 Aug., 1992.

Comparison of Elastic Scattering Cross Sections for Pions from ${ }^{3} \mathrm{H}$ and ${ }^{3} \mathrm{He}$ in the Backward Hemisphere, S. K. Matthews, B. L. Berman, W. J. Briscoe, R. W. Caress, K. S. Dhuga, S. N. Dragic, D. Knowles, D. Macek, N. J. Nicholas, M. F. Taragin, D. B. Barlow, B. M. K. Nefkens, C. Pillai, J. Price, L. D. Isenhower, M. E. Sadler, S. J. Greene, I. Slaus and I. Supek, submitted to Int. Nucl. Phys. Conf., Wiesbaden, Germany, 26 July-1 Aug., 1992.

Measurements of Differential Cross Sections for Pion-Nucleon Charge Exchange at Fonward Angles, N. J. Nicholas, W. J. Briscoe, M. F. Taragin, A. Mokhtari, M. E. Sadler, D. Isenhower, I. Supek, V. McVeigh, R. Towell, L. Towell, D. Lane, M. Brooks and A. Donley, submitted to Int. Nucl. Phys. Conf., Wiesbaden, Germany, 26 July-1 Aug., 1992.

Baryon Resonance Studies at PILAC, M. E. Sadler, PILAC User's Meeting at the Spring Meeting of the APS, Washington DC, 24 April 1991.

Analysis of the Low-Energy $\pi p \rightarrow \pi^{\circ}$ Experimental Data Taken in Light of Contemporary Theories, B. M. Brooks, M. A. K. Lodhi, T. J. Black, L. D. Isenhower, D. W. Lane, M. E. Sadler, J. D. Bowman and D. H. Fitzgeralid, Fall meeting of Texas Section of the APS, Texas City, 8-10 November, 1990.

Elastic Pion Scattering from ${ }^{3} \mathrm{H}$ and $3 \mathrm{He}$ at Backward Angles, S. K. Matthews, B. L. Berman, W. J. Briscoe, R. W. Caress, K. S. Dhuga, S. N. Dragic, D. Knowles, D. Macek, A. Mokhtari, N. J. Nicholas, M. F. Taragin, A. Donley, L. D. Isenhower, K. Powell, M. E. Sadler, D. B. Barlow, B. M. K. Nefkens, J. Price, W. R. Gibbs, B. F. Gilison, S. Greene and I. Supek, Bull. Am. Phys. Soc. 35, 945 (1990). 
-

$=$
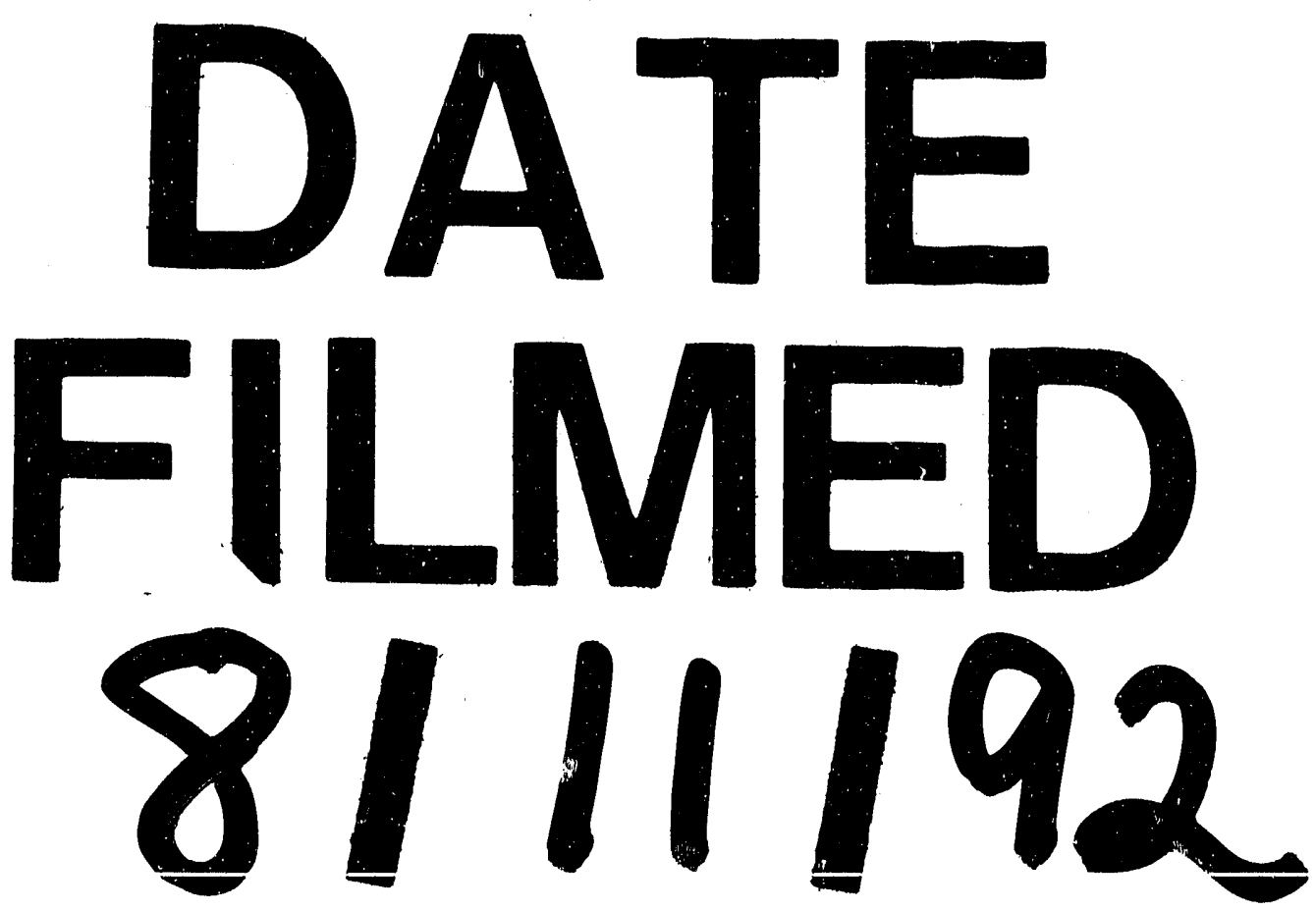
